\begin{abstract}
Title of Document:

REDUCTION OF SKIN STRETCH INDUCED

MOTION ARTIFACTS IN

ELECTROCARDIOGRAM MONITORING

USING ADAPTIVE FILTERING

Yan Liu, Doctor of Philosophy (Ph.D.), 2007

Directed By:

Chair Professor and Director, Michael G. Pecht, Department of Mechanical Engineering
\end{abstract}

Cardiovascular disease (CVD) is the leading cause of death in many regions worldwide, accounting for nearly one third of global deaths in 2001. Wearable electrocardiographic cardiovascular monitoring devices have contributed to reduce CVD mortality and cost by enabling the diagnosis of conditions with infrequent symptoms, the timely detection of critical signs that can be precursor to sudden cardiac death, and the long-term assessment/monitoring of symptoms, risk factors, and the effects of therapy. However, the effectiveness of ambulatory electrocardiography to improve the treatment of CVD can be significantly impaired by motion artifacts which can cause misdiagnoses, inappropriate treatment decisions, and trigger false alarms. Skin stretch associated with patient motion is a main source of motion artifact in current ECG monitors. A promising approach to reduce motion artifact is the use of adaptive filtering that utilizes a measured reference input 
correlated with the motion artifact to extract noise from the ECG signal. Previous attempts to apply adaptive filtering to electrocardiography have employed either electrode deformation or acceleration, body acceleration, or skin/electrode impedance as a reference input, and were not successful at reducing motion artifacts in a consistent and reproducible manner. This has been essentially attributed to the lack of correlation between the reference input selected and the induced noise.

In this study, motion artifacts are adaptively filtered by using skin strain as the reference signal. Skin strain is measured non-invasively using a light emitting diode (LED) and an optical sensor incorporated in an ECG electrode. The optical strain sensor is calibrated on animal skin samples and finally in-vivo, in terms of sensitivity and measurement range. Skin stretch induced artifacts are extracted in-vivo using adaptive filters. The system and method are tested for different individuals and under various types of ambulatory conditions with the noise reduction performance quantified. 


\title{
REDUCTION OF SKIN STRETCH INDUCED MOTION ARTIFACTS IN ELECTROCARDIOGRAM MONITORING USING ADAPTIVE FILTERING
}

\author{
By
}

Yan Liu

Dissertation submitted to the Faculty of the Graduate School of the University of Maryland, College Park, in partial fulfillment of the requirements for the degree of

Doctor of Philosophy 2007

Advisory Committee:

Professor Michael G. Pecht, Chair

Professor Mohammad Modarres

Professor Alison Flatau

Professor Patrick McCluskey

Professor Miao $\mathrm{Yu}$ 
(C) Copyright by

Yan Liu

2007 


\section{Acknowledgements}

With a deep sense of gratitude, I want to express my sincere thanks to my advisor Professor Michael Pecht for the great opportunity to work on this interesting topic. I am very thankful for his support, advice and encouragement. I feel very lucky to have an advisor who has great vision and insights to explore new research areas. In addition, his high reputation, always being energetic on work, and effective management skills have made him a perfect leader and career model for us. I also appreciate the training of technical presentations in CALCE, which is very valuable for my career development.

I would like to thank Dr. Valerie Eveloy for spending a lot of time on many technical discussions, and for thoroughly reviewing my papers. I also want to thank Dr. Diganta Das and Dr. Michael Azarian, who gave me numerous good suggestions on my presentations. These comments are very helpful for completing this project.

A lot of good suggestions were provided on my dissertation by Professor Alison Flatau, Professor Mohammad Modarres, Professor Patrick McCluskey, and Professor Miao Yu, and I am grateful to all of them.

I am thankful to all the members in my group, Nikhil Vichare, Haiyu Qi, Yuliang Deng, Bo Song, Vinh Khuu, Reza Keimasi, Sheng Zhan, Jie Gu, Anupam Choubey, Dr. Keith Rogers, Bhanu Sood, Anshul Shrivastava, Dr. Sanka Ganesan, Dr. Ji Wu, Dr. Peter Rodgers, Chris Wilkinson, Sony Mathew, Yuki Fukuda, Ping Zhao, Tong Fang, Sanjay Tiku, Dan Donahoe, Brian Tuchband, Shirsho Sengupta, Shunfeng Chen, Lei Nie, Weiqiang Wang, Snehaunshu Chowdhury, Matthias 
Ehrmann, Johann Wunsch - thank you for your encouragement and advices. You guys are very talented and it has been a pleasure to work with you.

I want to especially thank my husband Tao Jin for always supporting and encouraging me in tough times. I also appreciate my parents for always standing by me these years that I feel so blessed. There are many other friends who always cared about my study and encouraged me a lot. Though I didn't name them one by one here, I want to thank them all for their kindly support. 


\section{Table of Contents}

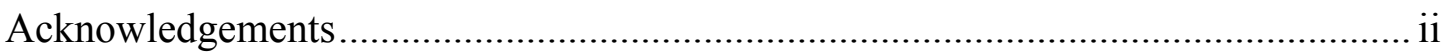

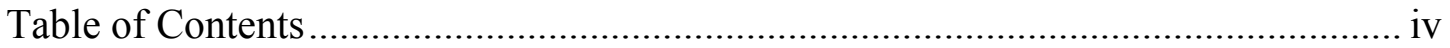

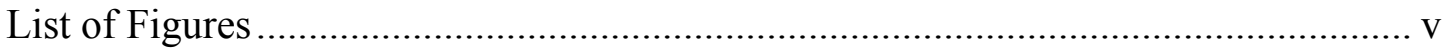

Chapter 1: Non-invasive Ambulatory Monitoring of Cardiovascular Disease............. 1

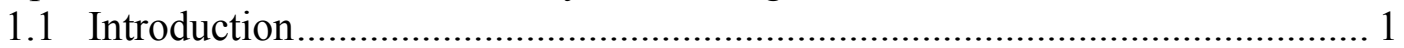

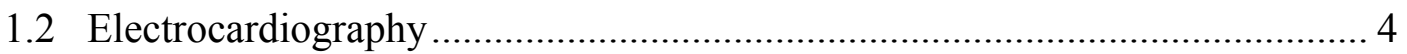

1.3 Blood Pressure Monitor ............................................................................. 13

1.4 Photoplethysmograph and Pulse Oximeter .................................................. 18

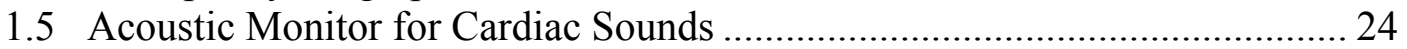

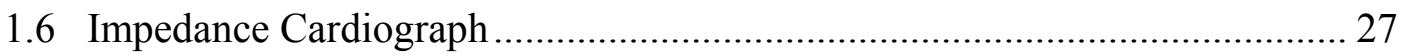

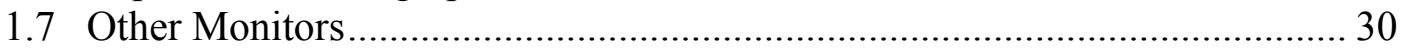

1.8 Noninvasive Multiple Sensors Monitoring Systems.......................................... 32

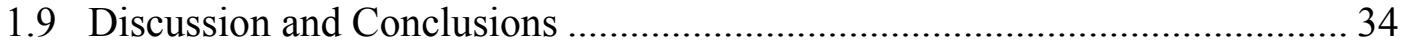

Chapter 2: Reduction of ECG Motion Artifacts ........................................................ 37

2.1 Motivation / Problem Statement ................................................................. 37

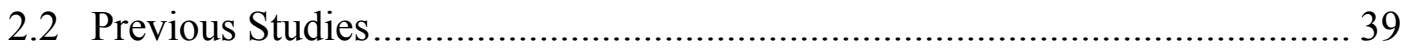

2.2.1 Skin Stretch - the Main Source of ECG Motion Artifact.......................... 40

2.2.2 Reduce Motion Artifact by Skin Abrasion and Puncturing ...................... 43

2.2.3 Reduce Motion Artifact by Adaptive Filtering ........................................ 44

2.3 Research Objective ................................................................................. 49

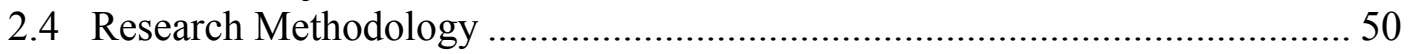

Chapter 3: Optical Sensor Calibration and Prototyping …………………………..... 52

3.1 Integration of an Optical Sensor into an ECG Electrode ................................. 52

3.2 Calibration of the Optical Skin Stretch Sensor .............................................. 56

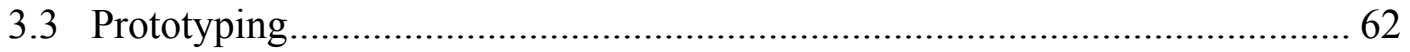

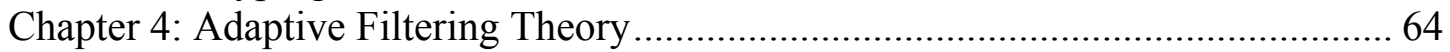

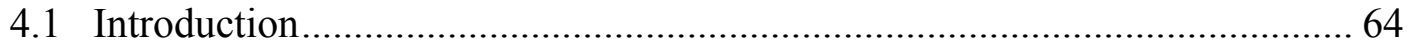

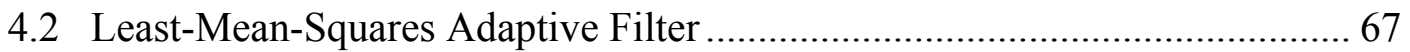

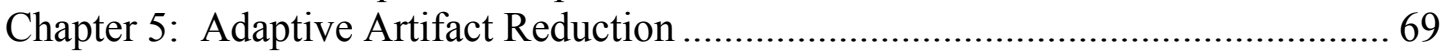

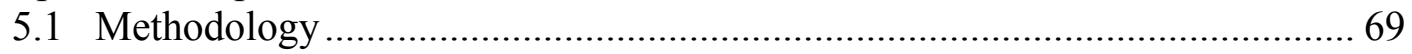

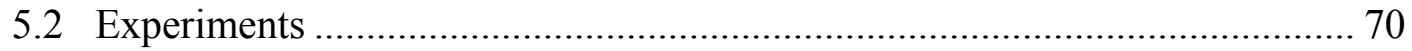

5.3 ECG Artifacts Reduction ...................................................................... 76

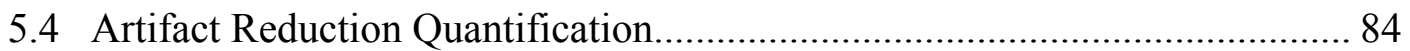

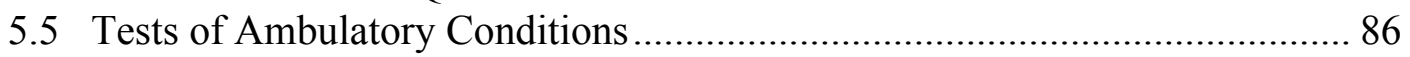

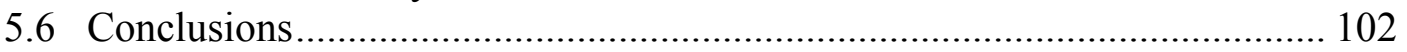

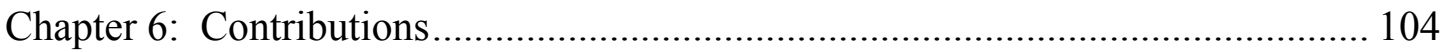

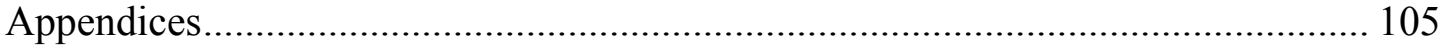

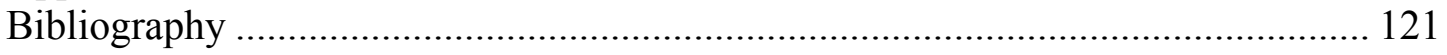




\section{List of Figures}

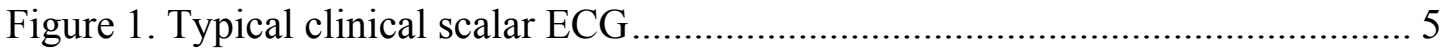

Figure 2. (a) ECG signal without artifact; (b) ECG signal with notion artifact.......... 39

Figure 3. Schematic diagram of the skin (Tam and Webster 1977) ......................... 41

Figure 4. Thakor and Webster's (1978) injury current model of epidermis ............... 43

Figure 5. Adaptive filter structure ...................................................................... 45

Figure 6. Measurement of electrode motion using an accelerometer (Tong 2000) .... 46

Figure 7 . Block diagram of the cause and effect relationship between variables and

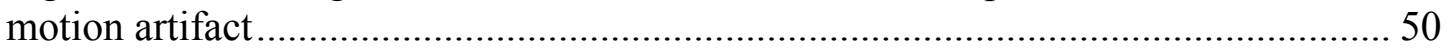

Figure 8. Electrode/ sensor system and adaptive filter structure to reduce ECG motion

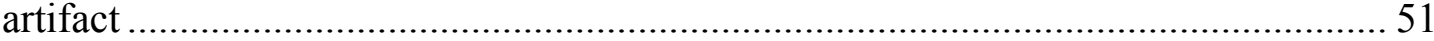

Figure 9. Isometric view (left) and exploded isometric view (right) of an optical

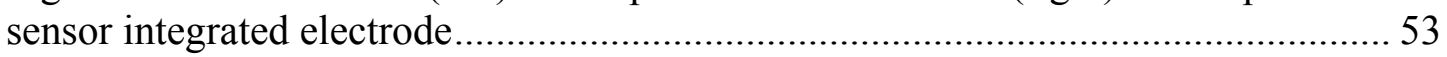

Figure 10. Optical components integrated in an electrode ................................... 54

Figure 11. Illuminated skin surface area underneath the CMOS image sensor.......... 55

Figure 12. The optical sensor identified common features in sequential images to determine the direction and amount of relative displacement of the area underneath the sensor. Image (b) was take a short time after image (a), while there is relative movement between the sensor and the area beneath it (Avago Technologies 2005). 56

Figure 13. In-vitro Calibration of the optical stretch sensor .....................................5

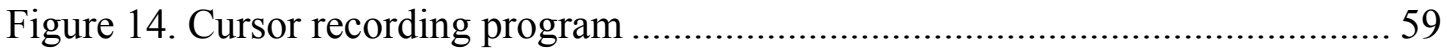

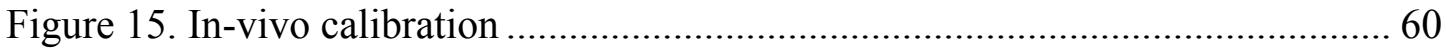

Figure 16. Optical sensor output versus the strain of an animal skin specimen ......... 61

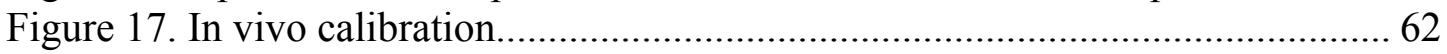

Figure 18. Optical sensor output vs. skin strain with different L (distance between the

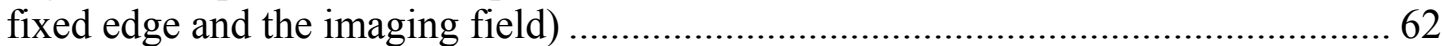

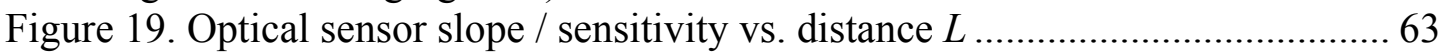

Figure 20. Electrode-sensor system and adaptive filter structure to reduce ECG

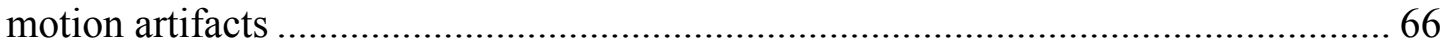

Figure 21. Apparatus for (a) ECG monitor; (b) ambulatory ECG monitor ................ 70

Figure 22. Lead II ECG and skin strain measurement........................................ 72

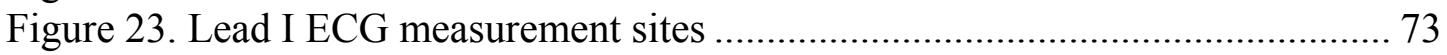

Figure 24. The ECG amplifier, electrodes and the optical sensor ........................... 74

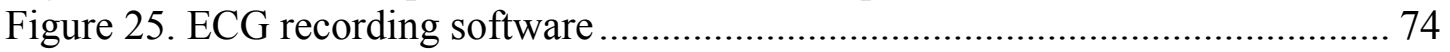

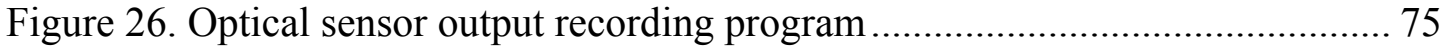

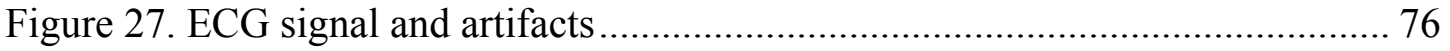

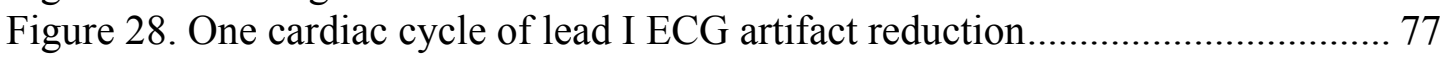

Figure 29. Artifact reduction of a 30 -second lead I ECG data ............................... 78

Figure 30. Lead II ECG and skin strain induced artifacts....................................... 79

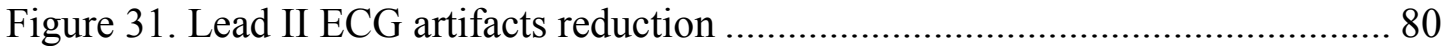

Figure 32. (a) Artifact-free ECG and ECG with artifacts for subject a; (b) Artifact-

free ECG and ECG with artifacts for subject b............................................. 82

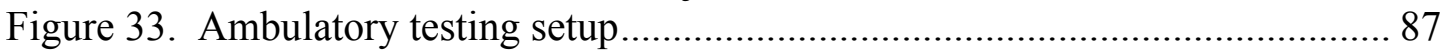

Figure 34. Ambulatory ECG motion artifact reduction (recording 1: subject is slightly stretching the upper chest) 
Figure 35. Ambulatory ECG motion artifact reduction (recording 6: subject is raising

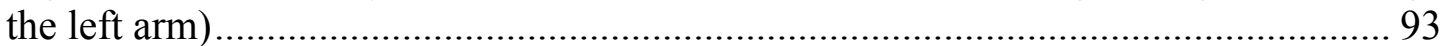

Figure 36. Ambulatory ECG motion artifact reduction (recording 7) f....................... 94

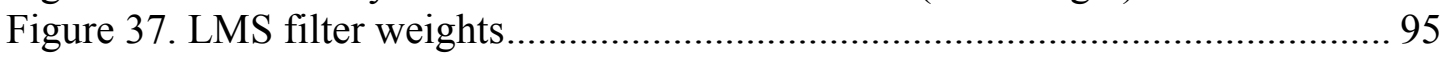

Figure 38. LMS filter performance …………………………………………...... 96

Figure 39. LMS filter performance vs. filter order .................................................. 97

Figure 40. Ambulatory ECG motion artifact reduction. ECG was recorded when the subject is slightly stretching the upper chest (recording 1). First trace: skin strain signal; second trace: estimated artifacts; third trace: ECG with artifacts; fourth trace: ECG after artifact reduction.

Figure 41. Ambulatory ECG motion artifact reduction (recording 6: subject is raising the left arm)

Figure 42. Frequency domain analysis of artifact-free ECG (first trace), ECG with artifacts (second trace), and ECG after artifact reduction (third trace). The top trace is recorded when the subject was at rest. The second and third traces were taken when the subject was raising the left arm. 101 


\section{Chapter 1: Non-invasive Ambulatory Monitoring of Cardiovascular Disease}

\subsection{Introduction}

Cardiovascular disease (CVD) is the leading cause of death in many regions worldwide, accounting for nearly one third of global deaths in 2001 (American Heart Association 2005b), and as much as $40 \%$ of deaths in the United States (US) and the European Union (EU) (American Heart Association 2005a, Petersen et al. 2005). In the US, when considered as either a primary or contributing cause, CVD mortality represents nearly $60 \%$ of all mortality (American Heart Association 2005a). In addition, $32 \%$ of deaths from CVD are premature deaths (i.e., before the average life expectancy) (American Heart Association 2005a). The total yearly cost of CVD has been projected to reach US $\$ 394$ billion in the US this year (American Heart Association 2005a), and is currently estimated to be over US $\$ 169$ billion in the EU (Peterson et al. 2005).

The two most frequent forms of CVD are coronary heart disease (CHD) ${ }^{1}$ and stroke (also termed cerebrovascular disease), which alone cause $53 \%$ and $18 \%$ of CVD-induced deaths in the US, respectively (American Heart Association 2005a). Other common types of CVD include congestive heart failure ${ }^{2}$, high blood pressure,

\footnotetext{
${ }^{1}$ Coronary heart disease involves a reduction in the blood supply to the heart muscle by narrowing or blockage of the coronary arteries (atherosclerosis). In time, inadequate supply of oxygen-rich blood and nutrients damages the heart muscle and can lead to myocardial infarction (heart attack) and angina pectoris (chest pain).

${ }^{2}$ Condition resulting from weakness of the heart muscle, in which the heart cannot pump out all of the blood that enters it. This results in an accumulation of blood in the vessels leading to the heart and fluid in various parts of the body such as the lungs, legs, and abdomen tissues.
} 
and diseases of the arteries (American Heart Association 2005a). Such conditions are chronic diseases, or wearout mechanisms from a reliability engineering viewpoint.

The elderly (age 65 or older) carry a higher risk of CVD-induced death compared with the rest of the population, and represent $39 \%$ of all patients affected by CVD in the US (American Heart Association 2005a). The aging of the population, which will lead to 40 million Americans being aged 65 or older by 2010 (American Heart Association 2005a), will result in an increased incidence of chronic CVDs. CVD also represents the second largest cause of death in the US population aged less than 15. While most CVDs in children are due to congenital cardiovascular malformations, an increasing number of children are developing risk factors including high blood pressure, smoking, high blood cholesterol, physical inactivity, overweight, and the metabolic syndrome ${ }^{3}$. Furthermore, it is anticipated that cardiovascular complications resulting from other conditions, namely obesity and type 2 diabetes, which are affecting a growing portion of the population, will contribute to further fuel the cardiovascular outbreak for years to come (American Heart Association 2005a). In this context, progress in early, accurate and cost-effective diagnosis and assessment of chronic CVDs, and their prevention, are becoming critical to improve life expectancy and reduce the economic toll of CVD. It is estimated that if all forms of major CVD were eliminated, life expectancy in the US would rise by almost seven years (American Heart Association 2005a).

Although current medical and clinical approaches of CVD assessment can provide direct and accurate evidence of heart disease, these techniques only provide

\footnotetext{
${ }^{3}$ Abnormal levels for three or more of the following factors: waist

circumference, serum triglyceride, high-density lipoprotein (HDL) cholesterol, blood pressure and fasting glucose level.
} 
periodic (intermittent) assessment of the disease. Considering that the majority of cardiac deaths are sudden deaths, which occur before the patient can reach a medical care facility (Center for Disease Control and Prevention 2002), there is a need for outpatient continuous CVD monitoring to enable the timely detection of precursor symptoms to sudden death, and to enable the long-term management of chronic conditions through monitoring of symptoms, risk factors, and regimen compliance. Conversely, a large portion of patients surviving a heart attack require potentially risky and costly invasive procedures such as coronary angioplasty ${ }^{4}$ or coronary artery bypass surgery. Such procedures could be avoided by continuous CVD monitoring, which could prevent hospitalization through the timely detection of critical symptoms.

Clinical techniques such as echocardiography and MRI are operationally complex and require physically large equipments, and are therefore not suitable for use in rural areas, primary healthcare facilities and homecare (Pavlopoulos et al. 2004). Portable or wearable monitoring cardiac devices can supplement traditional medical care by assisting the follow up and treatment of patients with heart disease, particularly the elderly who require more frequent cardiac assessment, and for whom traveling to a clinical care facility may be difficult and costly. The use of cardiac health monitoring has also been proposed for professional categories whose access to medical care is restricted by geography or environment (Scanlon 1998).

This chapter assesses current and emerging non-invasive ambulatory CVD monitoring techniques. The requirements, challenges, applications, and perceived

\footnotetext{
${ }^{4}$ Invasive procedure performed to reduce or eliminate blockages in coronary arteries. A catheter is guided into the clogged heart artery from an artery in the leg or arm. An expandable balloon is passed to the blockage and inflated to push against the plaque and open the artery. The balloon is then deflated and the catheter withdrawn.
} 
medical value of the monitoring technologies presented are analyzed. Ambulatory electrocardiographic and blood pressure monitors are generally considered the most mature technologies (Liu et al. 2005). While sensing techniques for blood perfusion, oxygen saturation, cardiac sound, and vascular blood velocity have been implemented in standard clinical devices, ambulatory applications are still at early prototyping phase. Multiparameter cardiovascular monitors, typically in the forms of clothing, have recently appeared and are introduced.

\subsection{Electrocardiography}

The electrocardiogram $(E C G)^{5}$ is currently the most widely prescribed medical diagnostic procedure. The main diagnostic applications of the clinical ECG are arrhythmia ${ }^{6}$, cardiac myopathies ${ }^{7}$, and coronary blood flow abnormalities (Guyton and Hall 2000). In addition, electrocardiography is also used to evaluate the effects of medication and monitor patients during surgery.

A typical ECG waveform is shown in Figure 1, and is composed of a P wave, a QRS complex (which is comprised of Q, R and S waves), and a T wave (Guyton and Hall 2000). The $\mathrm{P}$ wave is produced by atrial depolarization, the QRS complex primarily by ventricular depolarization, and the $\mathrm{T}$ wave by ventricular repolarization (Webster 1998). The significant features of the waveform are the waves and their individual durations, and time intervals including the P-R, S-T and Q-T intervals

\footnotetext{
${ }^{5}$ An electrocardiogram (ECG) is a graphic recording of the body-surface electrical potentials generated by electrical activation of the ventricular and atrial muscles (Webster 1998). Their activation involves sequential polarization and depolarization of the myocardial cells, that produce closed-line action currents that flow in the thoracic volume conductor (Webster 1998). A small portion of the currents spreads to the surface of the body.

${ }_{7}^{6}$ Abnormal heart rhythm.

${ }^{7}$ Any of various abnormal conditions or diseases of the cardiac muscular tissues (fibres).
} 
(Webster 1998). The amplitude of the ECG is normally 0.1 to $3 \mathrm{mV}$, with a frequency range of 0.05 to $100 \mathrm{~Hz}$ (Smith 1984).

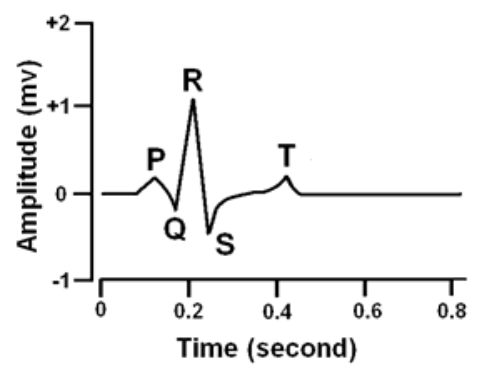

Figure 1. Typical clinical scalar ECG

In electrocardiography, the difference in electrical potential between specific sites on the skin is measured using electrodes (Guyton and Hall 2000) that transduce cutaneous ionic current into electronic current to measure biopotentials (Webster 1998). The electrodes are typically metal plate, silver/silver chloride $(\mathrm{Ag} / \mathrm{AgCl})$ electrodes that are attached to the skin using adhesive pads. An electrolyte paste or gel containing chloride anions is used to establish electrical contact between the electrode and the skin (Webster 1998).

Clinical and ambulatory electrocardiographs differ in terms of the number of leads ${ }^{8}$, and data storage, processing, transmission, and reporting format. Clinical electrocardiographs employ twelve leads, usually six in the frontal plane ${ }^{9}$ and six in the transverse plane ${ }^{10}$ (Webster 1998), with a total of ten electrodes. Advances in signal processing techniques and computational power have enabled the use of

\footnotetext{
${ }^{8}$ Pair of electrodes used to measure the potential difference between two sites.

${ }^{9}$ Plane of the body parallel to the ground when the patient is laying on his/her back. The six frontal leads consist of three basic leads (left arm to right arm, left leg to right arm and left leg to left arm) that form Eindhoven's triangle, and three augmented leads (also referred to as Golberger's leads), which are functions of the three basic leads (Webster 1998).

${ }^{10}$ Plane of the body parallel to the ground when the patient is standing plane of the body parallel to the ground when the patient is standing. The six transverse leads are six precordial (chest) leads that each consist of an electrode referenced to a common potential (Webster 1998).
} 
computerized ECG interpretation. Although useful for the precise calculation of certain ECG characteristic parameters, the overall diagnostic accuracy of such interpretation programs still requires improvement (Kadish 2001).

Ambulatory ECG monitors can be classified as either continuous (Holter monitors) or intermittent recorders (ECG event monitors or episodic monitors) (Kadish 2001, Kowey and Kocovic 2003, Zimetbaum and Josephson 1999). Their main application is the diagnosis of intermittent $\operatorname{arrhythmia}^{11}$ that can remain undetected in a clinical examination, since certain abnormalities may occur only during sleep or with mental, emotional, or exercise induced changes in cardiac oxygenation or function (Kadish 2001). Ambulatory ECG monitors also permit the occurrences of abnormal cardiac electrical behaviour to be characterized while the patient is pursuing his/her normal activities, which is not feasible by clinical electrocardiography. It has been estimated that of the 88 electrocardiographic diagnoses that can be made from a 12-lead ECG, up to 63 can be performed using ambulatory electrocardiography (Kadish 2001). In terms of prognosis, Zimetbaum and Josephson (1999) note that guidelines are lacking on the use of ambulatory monitors for prognosis in patients at potential risk of sudden arrhythmic death.

The Holter monitor was developed in the 1960's to continuously record ECG data for 24 to 48 hours. Apart from the detection of intermittent arrhythmic symptoms, continuous monitoring is also used to assess antiarrhythmic or concomitant drug therapy ${ }^{12}$ response, to survey patients with implanted pacemakers or cardioverter defibrillators (ICDs), including potential device failure, and can assist

\footnotetext{
${ }^{11}$ Abnormal heart rhythm.

${ }^{12}$ Simultaneous drug therapy.
} 
in the screening and therapy of silent myocardial ischemia ${ }^{13}$ (Kadish 2001, Virginia Mason Medical Center 2000). The number and position of the electrodes varies with the model, but most Holter monitors employ two to three leads attached to the chest (Kowey and Kocovic 2003). The data is recorded on either a tape, flash card or disk, with no patient activation required, and is analyzed offline (Kowey and Kocovic 2003). The patient records his/her activities and any detectable symptoms (e.g., faintness, dizziness) in a diary, so that this information can be subsequently correlated with the ECG data. The main limitation of the traditional Holter monitor is that the recording time can be too short to capture infrequent arrhythmia, due to memory constraints (Zimetbaum and Josephson 1999). It is estimated that in general, only $10 \%$ of arrhythmias that are suspected from symptoms are detected (Kowey and Kocovic 2003). Extending the period of observation requires serial recordings, which is currently considered impractical and $\operatorname{costly}^{14}$ (Kowey and Kocovic 2003). In addition, the recorder size can interfere with normal activities and prevent exercise that may trigger rhythm abnormalities to be detected (Zimetbaum and Josephson 1999). Also, patients frequently forget to document the timing of their symptoms, which can impact the specificity of the ECG interpretation (Zimetbaum and Josephson 1999).

ECG event monitors serve to evaluate infrequent symptoms via continuous monitoring over periods of up to three months, but recording is intermittent (Kadish 2001). Event monitors can be classified as either post event recorders, loop recorders, or mobile cardiac outpatient telemetry systems. In traditional post event recorders,

\footnotetext{
${ }^{13}$ Inadequate supply of oxygen-rich blood to the heart that does not cause symptoms such as chest pain.

${ }^{14}$ A Holter monitor (hardware) typically costs several thousand US dollars (MedFirst Healthcare Supply, Inc.). Patient cost for 24 hours monitoring is estimated at 353 US dollars (Zimetbaum and Josephson 1999).
} 
recording is manually triggered when abnormal symptoms are detected by the patient. The recorded ECG is stored and subsequently transmitted by telephone to a base station. Such monitors can combine one pair of electrodes (one lead) with the recorder in a single unit, in the form of a credit card-size patch (Charuvastra 1998) that can be carried in the pocket and held to the chest for recording, or wrist watch type device (Mona-System Ltd., LifeWatch 2004). Wrist watch recorders have one electrode on the back of the watch and the other electrode beside the display, with the circuit completed by the application of the opposite hand over the display-side electrode. Such systems provide up to ten or fifteen minutes single channel recording, and the data can be transferred to a personal computer (PC) via an optical cable or standard modem (Mona-System Ltd., LifeWatch 2004). Concerns have been expressed that the quality of the ECG signal recorded by wrist watch type event monitors, can be inconsistent and unreliable (Charuvastra 1998). A major limitation of post event recorders is their inability to capture the initiation of or the period directly preceding the onset of arrhythmia (Zimetbaum and Josephson 1999), since triggering the data recording can be difficult for the patient when a symptom is in progress, particularly for elderly patients (Zimetbaum and Josephson 1999). Continuous-loop event recorders address this shortcoming by continuously recording and erasing electrocardiographic data, so that data measured from one to four minutes before, and 30 to 60 seconds after the device was activated can be stored (Kowey and Kocovic 2003). This technology can enable very fleeting symptoms, tachycardia ${ }^{15}$ onset, and in some cases, infrequent syncope ${ }^{16}$, to be captured (Kadish 2001). The

\footnotetext{
${ }^{15}$ Rapid heartbeat (typically in excess of 100 beats per minute).

${ }^{16}$ Temporary loss of consciousness.
} 
most common type of continuous-loop event monitors consists of a recorder (about the size of a beeper) that can be attached to the patient's belt and two patch disposable, waterproof precordial electrodes (Zimetbaum and Josephson 1999) that are typically changed every three to four days (Charuvastra 1998). Another solution is the use of automatically activated recorders, which employ algorithms to initiate recording upon the detection of an abnormal heart rhythm (Kowey and Kocovic 2003). A category of automatically activated recorders, referred to as mobile cardiac outpatient telemetry systems, are equipped with cellular-based transmission. Upon the detection of abnormal heart rhythm by embedded algorithms, ECG data are transmitted automatically to a monitoring center, along with any symptoms and activity data recorded electronically by the patient (Cardionet 2002, Cleveland Clinic Heart Center 2003). The system presented in (Cardionet 2002), for example, uses three wired precordial electrodes (i.e., two leads), and allows up to two weeks continuous monitoring. Although promising, this technology requires further validation to quantify its potential to improve both the number of arrhythmia episodes detected and the rapidity of medical response (Kowey and Kocovic 2003).

The use of current ECG monitors is generally considered too expensive, except for patients at high risk (Welch et al. 2004). Focusing on the reduction of hardware costs to expand the availability of such devices, Welch et al. (2004) developed a single lead, wireless, wearable ECG monitor that integrates two electrodes, a system-on-chip microcontroller for low-rate data sampling, algorithmic and filter-based detection of heart beats and selected arrhythmia, and a short range 
ISM band ${ }^{17}$ transceiver. The optimum position of the sensor was found to be a vertical orientation on the sternum. The monitoring time of the device is limited by battery life (a pair of disposable lithium cells having an expected life of three days), or the time that adhesives will maintain sensor attachment to the skin. The monitor is intended for heart rate recording and the detection of ventricular fibrillation ${ }^{18}$, ventricular tachycardia, and asystole ${ }^{19}$, as well as the assessment of pacemaker function. Preliminary evaluation of sensor performance in the presence of electrode motion and muscle artifact was found to be promising.

The performance of current electrocardiogram (ECG) monitors can be significantly degraded by motion artifacts, which is discussed in more depth in Chapter 2.

In recent years, new types of electrodes have been developed. These include the fabric electrodes developed by Paradiso et al. (2005) and Scilingo et al. (2005), and various types of dry electrodes, including the non-contact electrical potential probe developed by Prance et al. (2000) and Harland et al. (2002, 2003), the noncontact optical sensor developed by Kingsley et al. (2002, 2003, 2004) and Johnson (2003), and semi-invasive electrode using carbon nanotubes arrays developed by Ruffini et al. (2005). The fabric electrodes can be embedded in a garment. The dry electrodes have the advantage of easy applications, since the use of electrolytic gel and adhesive attachment is eliminated. These are in the development stage and haven't been used in clinical applications.

\footnotetext{
${ }^{17}$ Unlicensed band (902 to $928 \mathrm{MHz}$ ) intended for low-data rate industrial, scientific, and medical communications, which is widely used for short-range wireless data links.

${ }^{18}$ Abnormally rapid, disorganized and inefficient contraction of muscle fibres of the heart. This condition is life-threatening.

${ }^{19}$ Absence of electromechanical activity within the heart. This condition is life-threatening.
} 
Paradiso et al. (2005) and Scilingo et al. (2005) developed conductive fabric electrodes imbedded in the clothing to make a comfortable garment capable of recording ECG signals. The fabric electrodes were realized with stainless steel yarns twisted around a viscose textile yarn, with about $30 \%$ metal. A hydrogel membrane was used to improve the contact between fabric and skin, and to improve the electrical signal quality in dynamic conditions with the presence of ions in the membrane. Results showed that the signal obtained by the integrated systems is comparable with that obtained by standard electrodes.

As an alternative to the $\mathrm{Ag} / \mathrm{AgCl}$ electrodes that have relatively low impedance (106 to $10^{7} \mathrm{Ohms}$ ) and sensitivity, and require direct electrical contact with the skin through an electrolytic paste and adhesive attachment, Prance et al. (2000) and Harland et al. (2002, 2003) developed a high input impedance $\left(10^{15}\right.$ Ohms) electrical potential probe that detects a displacement current instead of a charge current, and uses feedback enhanced and stabilized electrometer based amplifiers. Such probes can be operated in either mechanical (but not electrical) contact mode whereby coupling with the body signal is formed via electrode-body interfaces, or remote off-body mode whereby the probe forms capacitive coupling with the body. In contact mode, both the probe noise floor level $\left(70 \mathrm{nV} \mathrm{Hz}^{-0.5}\right.$ at 1 $\mathrm{Hz}$ ), and inherent lack of cross coupling between closely spaced electrodes (as would occur between conventional electrical contact electrodes through leakage currents), enable the detection of low-amplitude biopotentials. Prance et al. (2000) and Harland et al. $(2002,2003)$ could measure detailed features of the ECG (namely, His-Perkinje discharge and $\mathrm{U}$ wave), which are typically not detected by a conventional 12-lead 
surface ECG. (The His-Perkinje discharge peak is generally measured using an intracardiac catheter). Other envisaged low level biopotential measurement applications include fetal heart beat $(10 \mu \mathrm{V}$ at a few $\mathrm{Hz})$ and electroencephalograms ${ }^{20}$ (10 to $40 \mu \mathrm{V}$ at a few $\mathrm{Hz}$ ). The use of a differential signal provided by two probes (e.g., attached to left and right hand fingers) was found to significantly reduce electromyographic and other body noise sources that can affect single probe measurements. The prototype monitor developed in (Harland et al. 2003) uses two probes individually mounted in two wristwatch type housings, a wireless radio transmitter and a receiver located at a distance of one to ten meters from the transmitter. Although promising, this technology requires further evaluation, including the susceptibility to artifacts. In remote mode, (Harland et al. 2002) and (Prance et al. 2000) could measure heart beats at a distance of up to one meter from a clothed body, which could open up new biopotential sensing applications.

Another type of non-contact ECG sensor was developed by Kingsley et al. (2002, 2003, 2004) and Johnson (2003). The sensor, named Photrode ${ }^{\mathrm{TM}}$, is a miniature, optical chip that consists of a specially designed integrated optical circuit. The optical sensor uses photons to detect and measure the body's bioelectric voltages, including ECG and EEG (electroencephalogram). The output optical signal of a superluminescent diode enters the sensor, and the biopotential signal ECG or EEG changes the phase of the light propagating in the sensor and converts it to intensity modulation. The modulated light is conveyed by optical fibre to an optical receiver, where the ECG or EEG signal is converted back to the electronic regime, and interpreted with signal processing. ECG / EEG data obtained using Photrode ${ }^{\mathrm{TM}}$ is

\footnotetext{
${ }^{20}$ A recording of the electrical activity of the brain, obtained using electrodes attached on the scalp.
} 
claimed to comparable to that obtained from conventional electrodes. The main advantage of the optical sensor is to eliminate the need for troublesome electrode attachment, since the sensor does not require physical contact to the body and can be placed over the subject's clothing to measure ECG. The technology is especially well suited in functional magnetic resonance imaging (MRI) of the heart or brain, since the sensor system is not sensitive to electromagnetic interference. The major technical challenge includes interferometric fibre optic lead noise caused by minute optical reflections (Kingsley et al. 2002, 2003, 2004, Johnson 2003).

Ruffini et al. (2005) developed a dry electrode with the contact surface covered with an array of carbon nanotubes. The nanotubes form a brush-like structure and penetrate the outer layer of the skin, the stratum corneum, to acquire biopotential signal. The invasive effect is negligible since the size of the nanotubes is much smaller than the pores of the skin, and the nanotubes barely penetrate the outmost layer of the skin, which avoid contact with nerve cells in deeper skin layers. The application of the nanotube electrode can eliminate the need for skin preparation and gel application, but its biocompatibility needs further investigation.

\subsection{Blood Pressure Monitor}

Two types of blood pressure ${ }^{21}$ (BP) monitors are currently available on the market, namely self-measured and ambulatory BP monitors. Self-measured BP monitors require recording to be initiated by the patient, and are suitable for home

\footnotetext{
${ }^{21}$ In blood pressure measurement, the pressure of blood flowing through the blood vessels against the artery walls is recorded. A blood pressure reading consists of two values (e.g., $120 / 80 \mathrm{mmHg}$ ). The first value represents systolic blood pressure, and reflects arterial pressure during systole (part of the cardiac cycle during which the heart muscle contracts to force blood into the arteries). The second value is diastolic blood pressure, and represents arterial pressure during diastole (i.e., relaxation or filling phase of the cardiac cycle).
} 
use. Ambulatory BP monitors typically provide up to 24 hours of intermittent, automated BP recording, including during sleep states. The measurement frequency is normally every 15 to 30 minutes. The data (50 to 100 readings per 24 hours) stored in the monitor can be subsequently downloaded to a computer.

Ambulatory BP monitors have greatly contributed to research on hypertension $^{22}$. The most common application of BP monitoring is the diagnosis of white coat hypertension $^{23}$ (Appel et al. 2002, McGrath 2002, Marchiando and Elston 2003). Other applications include therapy monitoring and diagnostic applications including nondipping BP pattern, apparent drug resistance, hypotensive symptoms to medications, episodic hypertension, and autonomic dysfunction (Appel et al. 2002, McGrath 2002, Marchiando and Elston 2003). The majority of ambulatory BP monitors in the market mainly utilize one of two sensing mechanisms, namely the auscultatory or oscillometric method. Other methods include volume clamp method, tonometry method, and the use of the correlation of blood pressure and pulse velocity signal.

In the auscultatory method (McGrath 2002, Marchiando and Elston 2003) a cuff secured around the upper arm is inflated above systolic pressure, to totally occlude the brachial artery ${ }^{24}$. The cuff is slowly deflated, and systolic pressure is measured at the onset of the first Korotkoff sound ${ }^{25}$, which is detected through

\footnotetext{
${ }^{22}$ High blood pressure. Hypertension is a common risk factor for cardiovascular disease. Fifty million Americans have hypertension, which accounts for approximately 25\% of the adult US population (Appel et al. 2002).

${ }^{23}$ Condition in which the patient has elevated BP during medical examination, but otherwise normal BP. White coat hypertension is also called isolated clinic hypertension, non-sustained hypertension, or office hypertension. Patients with white coat hypertension represents 20 to $30 \%$ of the patients who have high BP during medical examination. However, white coat hypertension presents a significantly lower health risk than sustained BP elevation.

${ }^{24}$ Main artery of the upper arm, located on the inside of the arm.

${ }^{25}$ The Korotkoff sounds are attributed to blood flow turbulence.
} 
auscultation using a microphone placed downstream of the cuff. Diastolic pressure is determined when the Korotkoff sounds cease.

The cuff oscillometric method (McGrath 2002, Marchiando and Elston 2003) is based upon the detection of cuff pressure oscillations at the brachial artery that are caused by vibrations in the arterial wall. Both systolic and mean arterial pressure are directly measured, and used to calculate diastolic pressure using one of several algorithms, depending upon the manufacturer (Marchiando and Elston 2003). The cuff pressure at the onset of the rise in pressure oscillations is generally recorded as systolic pressure. The cuff pressure at the point of maximum amplitude of the cuff pressure oscillations corresponds to the mean arterial pressure.

In the volume clamp method (Penaz 1973, Molhoek et al. 1981), the pressure of a finger-mounted cuff is continuously adjusted to equal (follow) intravascular blood pressure, resulting in constant vascular volume. Arterial volume is measured by plethysmography ${ }^{26}$. BP monitors utilizing this sensing technique are referred to as Portapres and its corresponding non-portable product is called Finapres.

Tonometry is a non-invasive method to measure arterial pressure by applying an array of pressure transducers on the wrist. A pneumatic pump and bellows press the transducers on the skin surface which partially flattening an artery to a bone surface. This pressure is less than arterial pressure therefore the artery is not occluded. The transducer recording the largest amplitude cardiac pulsations is determined automatically from the array and used to record arterial pressure waveforms. The system is calibrated with systolic and diastolic measurements from a standard arm cuff oscillometric method (Sato et al. 1993, Colin). This method

\footnotetext{
${ }^{26}$ Measurement of the volume or change in volume of a portion of the body.
} 
requires the sensor to be fixed on the wrist during continuous recording since it is susceptible to motions and sensor position change.

In the pulse velocity method, pulse signals are detected from two different locations on the subject's body by impedance plethysmograph sensor (Peterson 2004) or optoelectric sensors (Chen et al. 2003, 2005). The elapsed time between the arrivals of the corresponding points of the pulse signal at these two locations is determined and used to derive blood pressure. Shaltis et al. (2004) developed a method of cuffless BP monitoring that combines two reflective photoplethysmograph $^{27}$ (PPG) sensors attached to the ring finger of each of the two hands, with a hydrostatic pressure reference approach to calibrate the sensors. The obtained compliance curve between vascular volume and transmural pressure is used to derive BP from the plethysmographic measurements.

In clinical practice, BP is normally non-invasively measured on the brachial artery using either the auscultatory or cuff oscillometric method. Consequently, the majority of medical studies of blood pressure have been conducted with BP measured at that site. Generally, the more peripheral the measurement site, the higher the systolic BP and the greater the difference between systolic and diastolic pressure (Braun.com 2000). Therefore, measurements obtained from fingers or wrists are not equivalent to those obtained using traditional upper arm measurements. For this reason wrist / finger monitors have not been widely used due to lack of knowledge on the interpretation of wrist / finger ECG data, and lack of methods to accurately correlate wrist / finger ECG data with upper arm data.

\footnotetext{
${ }^{27}$ Photoplethysmography (PPG), or photoelectric plethysmography is a noninvasive optical technique for measuring tissue blood perfusion (Veraart et al. 1994, Cheong and Smith 2003).
} 
Areas for improvement in ambulatory blood pressure monitoring include the effect of cuff size on BP measurement accuracy, motion and posture-induced artifacts, patient tolerance, and cost, which is typically several thousands dollars for the hardware (Appel et al. 2002).

For the majority of BP monitors in the market, which are utilizing either auscultatory or oscillometric method, the use of a sufficient cuff length and width is critical for adequate compression of the brachial artery, as insufficient cuff size can lead to overestimation of BP (Kmom 2003). Although the importance of using a correctly sized cuff has been documented for over twenty years, this is still one of the most common sources of measurement error encountered with obese patients (Kmom 2003). Ng and Small (1993) showed by both experimental and analytical analysis that cuff-arm compliance and bladder size interact to affect the pulse amplitude, hence oscillometric BP measurements. A numerical study (Cristalli et al. 1994) indicates that apart from cuff size, the periarterial pressure imposed by an external cuff also depends on various factors including elastic properties of the arm soft tissues, and arterial position. In addition, traditional BP monitors with cuffs can interfere with daily activities and sleep (Staessen et al. 1995, Asada et al. 2003).

The performance of portable BP monitors in ambulatory conditions, during physical exercise or for patients whose cardiac rate is irregular (e.g., as in arterial fibrillation) has not been fully characterized (Staessen et al. 1995, McGrath 2002). A generally acceptable rule is that a BP recording is not acceptable if less than $85 \%$ of the readings are usable (McGrath 2002). Methods investigated to reduce artifact include the use of a simultaneous ECG recording as a reference signal to identify and 
filter artifact-contaminated BP measurement segments (McGrath 2002, Protocol Systems Inc., Chittenden and Weaver 1988), and the use of fuzzy-logic-based recursive weighted regression algorithms (Lin et al. 2003).

The use of ambulatory BP monitors has been controversial (Appel et al. 2002). Concerns have been raised that ambulatory BP monitors may be overutilized (Appel et al. 2002). In general, medical practices do not consider it necessary to repeat ambulatory BP measurements more than once a year, but patient monitoring needs are highly patient specific and depend upon a number of factors (O'Brien et al. 2000). While the estimation of BP from multiple measurements has been found to be a more sensitive indicator of cardiovascular outcome than occasional recordings (O'Brien et al. 2000), the capability of BP monitors to provide information than can supplement clinical measurements has been questioned (Appel et al. 2002). Also, it is still unclear whether self-measured BP monitoring is a satisfactory and less expensive alternative to ambulatory BP monitoring. Consequently, insurers have been reluctant to reimburse costs for the use of ambulatory BP monitors (Appel et al. 2002).

\subsection{Photoplethysmograph and Pulse Oximeter}

Photoplethysmography (PPG) is used to indicate the volume change in blood perfusion by an optical method. The intensity of light generated by a light emitting diode (LED) is transmitted through or reflected by the tissue, with the received signal indicating the volume change in blood perfusion. There are two types of PPG, depending on whether the measured signal is reflected light or transmitted. 
The most important parameter for practical use of photoplethysmograph is the venous refilling time after finishing a standardized exercise of the calf muscles (Veraart et al. 1994). Photoplethysmography can also be used to diagnose arterial diseases in fingers and toes, and functional disturbance of blood flow, and has been widely used in commercial pulse oximetry to measure oxygen saturation. In addition, PPG can provide a means to measure peripheral blood pressure.

Pulse oximetry is a method of measuring oxygen saturation ${ }^{28}$ of the arterial blood based on the absorption of specific wavelength of light by a blood specimen. An oximeter detects hypoxia (lack of oxygen supply to tissues) before the patients become clinically cyanosed (the skin looks blue due to lack of oxygen supply). Audible alarms can be provided by programming (Fearnley 1995). Pulse oximetry is now a standard practice in anesthesiology indicating the patients' cardio-respiratory status, and has also been successfully used in intensive care and the recovery room (Welch et al. 1990, Hill and Stoneham 2000).

A pulse oximeter consists of a probe, which is attached to the patient's finger or ear lobe, and a microprocessor unit for displaying the oxygen saturation and the pulse rate (Hill and Stoneham 2000). Two light emitting diodes (LED's) are within the probe. One light is in the visible red spectrum $(660 \mathrm{~nm})$ and the other in the infrared spectrum (940 nm) (Hill and Stoneham 2000). The beams of light pass through the tissue to a photodetector and some light is absorbed by the blood and soft tissues. The amount of light absorbed by haemoglobin at each light frequency defers depending on whether it is saturated or desaturated with oxygen (Fearnley 1995, Hill

\footnotetext{
${ }^{28}$ Oxygen saturation is the average percentage of saturation of a population of haemoglobin (oxygen transporting blood cell protein) molecules in the blood.
} 
and Stoneham 2000). By calculating the absorption at the two wavelengths the proportion of oxygenated haemoglobin is computed (Hill and Stoneham 2000). Pulse oximeters are available in the market produced by Biox and Nellcor etc (Welch et al. 1990) and the portable devices have been commercialized.

Efforts are on-going to develop ambulatory PPG monitors, though to date none of these devices are commercialized. Ambulatory PPG monitors have the advantages of easy operating, small size and low cost. However, the diagnostic value for long term monitoring needs to be further studied.

In 1995 Yamashita et al. (1995) developed a ring type telemeter that allows continuous vital sign monitoring and wireless data transmission. The technique is based on radio telemetry of PPG signal detected at the finger on which the ring is applied, and automatic alarming. A telemetry transmitter was made in the shape of a finger ring so that it can be easily installed. PPG signals were detected at the finger by a pair of LED's and a photodiode installed in the ring. The signal was transmitted to a remote receiver located in a house using an FM carrier wave. At the receiver, if the interval of the pulse becomes shorter or longer than a predetermined value, or the pulse amplitude become weak, an alarm system is activated. The alarm system indicates an emergency by an alarm sound or by dialing an emergency telephone number automatically. It was found that the signal amplitude was largely dependent on the positions of the LED and a PD. Power consumption is also an important factor since it determines the dimension and the weight of the transmitter.

In 1998 Rhee et al. (1998) and Yang et al. (1998) developed a PPG ring sensor for twenty-four hour continuous ambulatory and telemetric monitoring of a 
patient's arterial blood volume waveforms and blood oxygen saturation, with data wirelessly transmitted to a computer by an embedded RF transmitter. LEDs with two different wavelengths, red and near infrared, as well as a photodiode were imbedded in the ring, where the technology of pulse oximetry was implemented for blood oxygen saturation monitoring. Signals were received and analyzed by a home computer. Rhee et al. (2001) and Asada et al. (2003) proposed several solutions to reduce noise due to an ambient light source and motion artifacts. A double ring design was developed to lower the influence of external force, acceleration and ambient light to minimize artifacts. In this design, the optical sensor unit was separated from the rest of the ring body that was much heavier. This was achieved by having two rings mechanically decoupled to each other with only a thin flexible cable connects the two rings. The inner ring holds the sensors units alone while the outer ring contains the other parts. This design alleviates the influence of external forces applied to the ring, and shields the sensor unit from ambient lighting (Rhee et al. 2001). Other artifact reduction solutions were also investigated. A transmittal PPG configuration was chosen since it is less susceptible to disturbance than reflective PPG configurations (Asada et al. 2003). An accelerometer was used as a motion reference sensor to recover signals corrupted by motion artifacts (Asada et al. 2004). A reflective photo diode was proposed to be used as a motion reference sensor for canceling the disturbance and noise, since it is susceptive to disturbances (Asada et al. 2003). In addition, local pressure was proposed to be applied to increase the amplitude of the pulsatile signal so as to improve the signal-to-noise ratio (Asada et 
al. 2003), since applying a certain magnitude of pressure on the skin surface will help attaining stable PPG signals (Rhee et al. 2001).

Another key issue for developing the compact, wearable ring sensor is to reduce the power consumption, since the determinant factor of the dimension and weight of the ring sensor is those of a battery cell to be used (Yang et al. 1998). Rhee's ring sensor used a CPU to exploit digital control and transmission techniques in order to perform reliable and flexible operations with reduced power consumption (Rhee et al. 2001). Optimal internal clock frequency was obtained to minimize power consumption by analyzing the total power consumption in relation to characteristics of individual components, sampling rate, and CPU clock speed (Rhee et al. 2001).

Spigulis et al. (a, b) developed a portable PPG prototype device to detect the blood volume pulsations of micro-vessels in the skin, which contains information of metabolism and thermoregulation. It consists of an optical contact probe with a reflection type PPG sensor, bio-signal amplifying / filtering circuit and a lap-top computer with developed software. Diodes are closely mounted on a soft plastic pillow and fixed onto the measurement site by means of a sticky band. With adjusted length, the band can measure PPG at the finger tip, or from different locations of the body, e.g., forehead, neck, forearm, and knee (Spigulis et al. a). The device had undergone several tests and clinical results were obtained by analyzing the signal shapes detected from different sites.

Branche et al. (2004) developed wearable reflective pulse oximeters incorporated in the helmet for military applications. Commercially available reflectance-type pulse oximeter sensors are typically attached to the skin using a 
double-sides disposable tape. In Branche's study, alternative sensor attachment techniques were investigated by utilizing the existing helmet suspension liner, adjustable harness, and chin strap to secure the sensor to the skin. Simultaneous measurements acquired from the forehead, chin, jaw and nape areas indicated that the forehead region provides the best location for obtaining reproducible readings. Preliminary results confirmed that it is possible to achieve reproducible data from a helmet-mounted reflectance pulse oximeter sensor without the need to use adhesive tape to secure the sensor on the skin or readjustment of the helmet straps between successive measurements.

Pulse oximeters are accurate in the range of oxygen saturation of 70 to $100 \%$ $( \pm 2 \%$ ), but less accurate under $70 \%$ as there are no control oxygen saturation values to compare the data (Fearnley 1995, Hill and Stoneham 2000). Pulse oximetry is less effective in critically ill patients because tissue perfusion may be too poor for the pulsatile signal to be detected (Hill and Stoneham 2000). Pulse oximeter readings are not accurate in some situations including when there is a reduction in peripheral pulsatile blood flow produced by peripheral vasoconstriction or peripheral vascular disease, and in the situation of venous congestion. The signal can also be interfered by ambient lights, vascular dyes, skin pigmentation, nail varnish, and motions that cause transient change in the distance between the LEDs and the detector (Fearnley 1995, Welch et al. 1990, Hill and Stoneham 2000). Modern technologies have utilized several methods to reduce the effects of interference. These include time division multiplexing and quadrature division multiplexing. Time division multiplexing, whereby the LED's are cycled: red on, then infrared on, then both off, 
many times per second, helps to eliminate background noise. In quadrature division multiplexing, the red and infrared signals are separated in phase rather than time and then recombined in phase later. Motion artifact or electromagnetic interference can be eliminated since it will not be in the same phase of the two LED signals once they are combined (Hill and Stoneham 2000).

In summary, research have been conducted on the development of ambulatory PPG monitors and pulse oximeters, focusing on device miniaturization, power consumption reduction and noise minimization. The clinical usefulness of the continuous ambulatory monitors still requires evaluation.

\subsection{Acoustic Monitor for Cardiac Sounds}

Heart acoustic emissions consist of short, impulse-like events, termed "heart sounds". These are produced at transitions between the different hemodynamic phases of the cardiac cycle, and other acoustic emissions that are generally of longer duration and may occur within any phase of the cardiac cycle, termed "murmurs".

Four heart sounds have been identified, referred to as first (S1), second (S2), third (S3) and fourth (S4) heart sound. The heart sounds are multi-component, nonstationary signals (El-Asir and Mayyas 2004, Wood and Barry 1995, Xu et al. 2001). Most cardiac sounds have spectra that lie in the range of 1 to $1000 \mathrm{~Hz}$ (Selig 1993), but certain murmurs have frequencies up to $1500 \mathrm{~Hz}$ (Akay et al. 1992). Abnormalities in S1 and S2 and the presence of S3 and S4 and certain murmurs can indicate CVD. 
Cardiac auscultation has been a fundamental diagnostic measure in medical practice, and remains the most cost-effective method of initial cardiovascular health evaluation. Efforts to develop a wearable, wireless stethoscope are still in progress.

Scanlon (1998) developed wired acoustic sensors that could be used in a wearable acoustic monitoring system of soldier's cardiac, respiratory and voice sounds, and footfalls. This could enable the monitoring of soldier's physiological status (e.g., injury, hypothermia, dehydration) by field medical personnel, and of manpower availability by military commanders. The sensors consisted of 1 to 1.6 inch diameter flexural piezoceramics in an aqueous gel coupling layer, which provided acoustic impedance matching with human tissue, and impedance mismatch with air for ambient noise reduction. The sensors were mounted using straps, harnesses and a helmet headband at the torso, neck and head. However, the separation of cardiac sounds and noise (in particular, footfalls) based on short time Fourier transform analysis and the filtering used was not satisfactory, even for heart beat measurement. Individual's tolerance to sensor mounting hardware was also identified an area for improvement, highlighting the need for miniaturized, wireless sensors. Wireless heart sound monitoring systems have also been proposed for realtime continuous monitoring of heart failure patients by home-based telecare (Mendoza and Tran 2002), or for controlling the exercise levels of rehabilitation patients (Kim and Jong 2001). Such systems enable remote health care personnel to be informed of a dangerous status by wireless data transmission.

Asada et al. (2001) described concepts to develop passive, batteryless MEMSbased miniaturized condenser microphones (less than $5 \mathrm{~mm}$ in size) for use in a 
wearable, wireless stethoscope/telephone system. The sensors would be adhesively skin-attached for continuous cardiac and respiratory sounds monitoring and behavioural monitoring (e.g., eating, drinking speaking, coughing, walking). The system would inductively transmit vital sounds (e.g., pulse rate, heart rate variability, respiration rate) and voice recorded at the neck, chest, and cheeks wirelessly to a cellular phone. The technical challenges identified included the reduction of noise created by body motion, for which a sensor array was considered, loss of acoustic signal due to possible de-adhesion between the sensor and skin, and possible skin irritation. Cho and Asada (2002) developed these concepts further for respiratory sound monitoring of asthma patients and pulmonary disease patients. The feasibility of the proposed inductive acoustic data transmission concept was computationally assessed. It was concluded that limitations in mutual inductance would limit the use of such a system to short range monitoring, but these limitations were not quantified.

The application of heart sound measurement to CVD monitoring in prototype ambulatory devices has been hampered in part by the difficulty of reducing environmental noise and other unwanted sound such as respiration sound and speech, the frequencies of which largely overlap heart sound signals. Noise reduction methods include filtering, digital signal processing techniques, and the use of multiple noise reference sensors (Wang and Wang 2003). Although both environmental and lung sounds can be separated from cardiac sounds using signal processing techniques such as wavelet analysis, signal separation still needs improvements (Hall et al. 2000). 
Substantial efforts have been invested in cardiac sound measurement and analysis. However, challenges of noise reduction (e.g., environmental, respiratory, vocal) in ambulatory monitoring devices, and improving the understanding of heart sound genesis and propagation for pathology diagnosis, remain. The majority of researchers have focused on the identification and extraction of the morphological characteristics of the heart sounds, rather than on the application of heart sound measurement to the diagnosis of cardiac diseases. Despite advances in digital signal processing, wireless data transmission and sensor technologies, the measurement of cardiac sounds in current commercially-available health monitoring devices remains confined to heart rate monitoring (Eveloy et al. 2005).

\subsection{Impedance Cardiograph}

Impedance cardiography (ZCG, or $\mathrm{ICG})^{29}$ records impedance changes occurring in synchrony with respiration and ejection of blood into the aorta by emitting and recording a high-frequency alternating current along the thorax. Heart rate (HR) and systolic time intervals can be directly determined from the signals. Stroke volume (SV) and cardiac output (CO) can be estimated with proper algorithms (Nakonezny et al. 2001).

There are two types of measuring systems. The first system uses four band electrodes: two around the neck, the third one corresponding to the apex of the heart, and the fourth one further in the caudal direction. The second system uses eight electrodes: four around the neck and the others at the lower thorax (Grimnes and

\footnotetext{
${ }^{29}$ Impedance cardiography is a safe and noninvasive technique suitable for assessing cardiac function by measuring impedance signal from the thorax.
} 
Martinsen 2000). The second system was found to have a better signal-to-noise ratio (Grimnes and Martinsen 2000).

The amplitude of the impedance change is about $0.5 \%$ of the basic impedance value. The signal must be corrected for the variations due to respiration (Grimnes and Martinsen 2000). The technique has the advantage of being simple, cost effective, and noninvasive (Morucci et al. 1996). For impedance cardiography, it is multiple parts rather than a single structure within the thoracic cavity that is responsible for the impedance change. The relative contributions do not seem to be constant on an interindividual basis, and these contributions vary in an unpredictable way in pathological conditions (Morucci et al. 1996). A strong theory is lacking for impedance cardiography and it is still open for research (Morucci et al. 1996).

Currently most studies of impedance cardiography are conducted for resting patients in the laboratory or clinical environment (Sherwood et al. 1998). Ambulatory impedance cardiography has been commercialized by MindWare. Research of ambulatory impedance cardiography monitoring devices was also conducted by several groups. Cybulski et al. (1995) constructed an ambulatory monitor and record the signal on a 20-MB PCMCIA (Personal Computer Memory Card International Association cards) Flash Memory Card. Recorded signal allows off-line data analysis and beat-to-beat evaluation of cardiac output, stroke volume, ejection time, preejection period, and heart rate. Ambulatory impedance cardiography monitors were also developed by Sherwood et al. (1998) and Nakonezny et al. (2001) independently. 
In Cybulski's monitor, the system has been checked in 80 healthy subjects in ambulatory conditions (Cybulski et al. 1995, 1997, 2000). Hemodynamic signals were recorded using the ambulatory monitor together with simultaneous collection of ECG signals to demonstrate the hemodynamic efficiency of pacing in arrhythmic patients (Cybulski et al. 1997). Using the ambulatory monitor hemodynamic variability was also studied for patients with atrial fibrillation, and the results showed that these patients had increased variability of stroke volume than that of healthy adults (Cybulski et al. 1999).

Artifacts can be caused by motion, respiration and speaking. In Cybulski's ambulatory impedance cardiography, the percentage of properly recognized heart cycles with respect to the estimated parameters varied from 20 to $80 \%$ during the day and from 75 to $90 \%$ at night. Speaking and vigorous movements will corrupt the signal and markedly lower the useful signal percentage. Raza et al. (1989) developed a digital high pass filter to eliminate respiration artifacts from impedance cardiographic signal. The filter automatically selects the cutoff frequency $(\mathrm{CF})$ as a function of the heart rate. Other studies on reducing artifact of impedance cardiograph are limited in processing received signals, without considering eliminating the influence of artifact sources.

For bioimpedance, multiple electrodes have to be attached to the neck and upper body, raising again the problem of patient tolerance for long term continuous monitoring. Future studies on improving signal stability under real ambulatory conditions will be important, since this technology provides good indications of cardiac mechanics. 


\subsection{Other Monitors}

Other monitors under development include the Doppler Necklace proposed by Awad and Asada (Awad and Asada 1999, Asada and Awad 1999), using an accelerometer to record Arterio-Oscillo-Gram in Pouladian and Golpayegani et al.'s study (2003, 2005), and using accelerometers to monitor motility and postures by Grossman.

Awad and Asada (1999) presented a conceptual Doppler Necklace utilizing the Doppler ultrasound technology ${ }^{30}$ to measure blood velocity. Piezoelectric ultrasound sensors were proposed to be mounted on the neck to continuously monitor blood flow in the common carotid artery (Awad and Asada 1999, Asada and Awad 1999). The centerline velocity in that artery contains information of cardiac performance, diastolic cerebral flow, and the time rate of change of the pressure pulse waveform. An array of equally spaced transducers was designed to provide wide sector coverage in the neck, to resolve the problem of vessel location uncertainty. The transducers were designed to be alternately driven and the one yielding highest velocity is considered to correctly target the centerline of the vessel, thus is kept in emitting mode. Adjacent transducers can also be excited according to the need. Thus a reliable measurement is possible despite motion and location uncertainty of the target vessel. The signal is transmitted to a computer and the blood velocity can be analyzed continuously.

\footnotetext{
${ }^{30}$ Doppler ultrasound technology has been used within clinical environment to measure blood velocity and require a special operator since the technique is complicated. When using this technique, a sinusoidal voltage excites a piezoelectric crystal to create an ultrasound beam. The beam intercepts the target vessel and the backscattered ultrasound, having a frequency shift (the Doppler shift) due to blood movement, is picked up by a receiving crystal.
} 
In Pouladian and Golpayegani's research $(2003,2005)$, an accelerometer was noninvasively over brachial artery to measure the vibrations and oscillations of the arteries. The measured acceleration signal was named arterio-oscillo-gram (AOG). Mean of normalized spectrual samples between 30 and $250 \mathrm{~Hz}$ was calculated and defined as a feature. It was found that the more coronary occlusions (more severity of atherosclerosis), the larger the feature. This method, claimed by Pouladian, has the potential of being a screening tool for diagnosing atherosclerosis. The test results showed that this method can detect CAD (coronary artery disease) with $70 \%$ accuracy, and non-CAD with $90 \%$ accuracy.

Grossman proposed to use accelerometers in an ambulatory multiple parameter monitoring system (LifeShirt) to precisely quantify daily physical activities and energy expenditure. Accelerometers have been widely used to monitor human posture and movement parameters, including step rate, step rate variability, rise time, postural orientation during sitting and standing, postural sway during quiet standing and the energy expenditure in each movement (Mathie et al. 2004). However, little progress is made to develop tools to help directly identify specific situations and activities that trigger dangerous cardiac events (Grossman). Since many diseases, including coronary artery disease, are typically associated with aberrant physical activity levels, distinctive patterns of activity and energy expenditure might be seen for certain disorders after applying ambulatory physical activity monitors, and may help to better characterize CV conditions (Grossman). 


\subsection{Noninvasive Multiple Sensors Monitoring Systems}

VivoMetrics developed a sleeveless vest with embedded sensors (inductive plethysmography sensors, ECG electrodes, and a accelerometer) and optional peripheral diagnostic devices (blood pressure monitor, pulse oximeter, etc.) that can monitor a wide range of physiological parameters including ECG, respiration, physical activity and posture, blood pressure, blood oxygen saturation, skin temperature etc. (VivoMetrics). Data is recorded in a PDA worn on the patients' belt or put into a pocket. The screen of the device allows patients' subjective input of symptoms and activities synchronized to the recorded signals. A rechargeable battery and an exchangeable flash memory card can provide up to 24 hours continuous recording. Data is uploaded to a personal computer, sent to the Data Center via internet, and then reviewed and analyzed in the Data Center including calibration, artifact screening, parameter extraction and trends. The system has been used in more than 1000 hospitals worldwide. The system need to be further validated to assess the efficiency of the automatic artifact screening algorithms and the accuracy of certain parameters measured during postural changes. In addition, the usefulness of several extracted parameters in ambulatory monitoring has not been demonstrated.

A monitoring vest was developed by Sundaresan Jayaraman's team at Georgia Institute of Technology and is now sold by the company Sensatex for detecting bullet wounds (Georgia Tech, Sastry 2004). The Georgia Tech Wearable Motherboard (Smart Shirt) provides a framework for the incorporation of sensing, monitoring and information processing devices. Plastic optical fibres are woven in the shirt and connected to a photodiode at one end and a laser at the other, providing the function 
of bullet detection. Pulses of light are detected constantly by the diode with any light interruption indicating the exact location of the bullet's entry (Sastry 2004). Sensors have been plugged into the Smart Shirt and attached to any part of the subject being monitored. Flexible data buses integrated in the vest transmit data to recorders (Georgia Tech, Sastry 2004).

A wearable human-computer interaction technique (MIThril) is under development in the MIT Media lab. The hardware platform combines body-worn computation, sensing and networking in a clothing integrated design. The software combines user interface elements and machine learning tools (MIT Media Lab).

Different from the above wearable network systems that have metallic cables woven into the garment and bulky sensor size, a cable-free wearable sensor system was proposed by Wade and Asada (2004). The network infrastructure is based on the use of conductive fabrics as the electrical transmission medium, so that metallic cables can be eliminated. Two conductive sheets serve as the medium for power and data transmission instead of cables in traditional designs (Wade and Asada 2004).

Building a monitoring shirt as a real "personal computer" can allow wider sensor location choices. Noninvasive monitoring systems integrated with multiple sensors are under development and have become a hot topic. However the fully functioned device with sensors and recorders is quite bulky and device miniaturization remains a challenge. 


\subsection{Discussion and Conclusions}

There is a need for continuous cardiovascular monitoring to enable the longterm, real time detection of critical signs, risk factors, effects of therapy, and symptoms precursor to sudden death. Non-invasive ambulatory cardiovascular monitors are receiving more attention as a means of addressing this need and supplementing traditional medical and clinical care.

The monitoring of many CVDs is complex as generally there is no single parameter that is sufficient to assess CV health, except for very specific CVDs (e.g., arrhythmia). Various kinds of noninvasive CVD monitors exist with different technology maturity levels. Ambulatory ECG and blood pressure monitors have been used in clinical practice and has improved health surveillance. While the value of ambulatory ECG monitoring is undisputed, particularly in the context of life threatening arrhythmia, the usefulness of ambulatory BP monitors has not been fully established. Ambulatory monitors of parameters except ECG and blood pressure are in the early development stage, including pulse oximeters, heart sound and bioimpedance monitors. For these parameters, clinical usefulness of the continuous ambulatory monitors need to be further evaluated. The majority of the efforts are devoted to the development of new devices, with few studies focused on the evaluation of their medical value. This indicates that the exploration stage is not complete for noninvasive ambulatory cardiovascular monitors.

Current CV monitoring approaches focus on the detection of specific diseases

and are weak on prognostic value. Commercialized Holters and blood pressure monitors typically provide 24 to 48 hours continuous recording. During this limited 
period, existing monitors are focusing on specific disease detection or certain parameter recording rather than year-long observation and trend analysis. Future health monitors may extend their ability to either detect CVD by monitoring symptoms, or predict CVD by monitoring risk factors. Longer term cardiovascular monitoring is less explored and it is not yet known whether it can provide valuable information compared to occasional examination by a doctor. For extended monitoring period, low power consumption, device miniaturization, and data storage or transmission capacity become critical.

Ambulatory cardiac monitors should provide stable signals during the patient's daily activities. Resistance to motion artifact remains a significant challenge for all types of ambulatory monitors. Efforts are ongoing to eliminate artifacts by improving hardware design and applying signal processing algorithms.

The recorder size of the existing ambulatory ECG monitors and blood pressure monitors is quite bulky and heavy. In addition, for Holter ECG monitors, cumbersome wires and patches on the chest make it uncomfortable to wear in long term usage. In general, inconvenience or complexity, lack of comfort in wearing the monitor, and high cost limit the wide acceptance of these devices.

Various studies have been devoted to the development of ring, watch, band, or garment based wearable monitors, to improve the patience tolerance. However, whether the commercial ambulatory monitors can provide accurate information needs to be scrutinized. For ambulatory blood pressure monitors, measurements obtained from a finger or wrist are not equivalent to those obtained using traditional upper arm measurements, which is the current clinical standard. Wide application of wrist or 
finger BP monitors will require additional data interpretation and correlations with upper arm data.

Although a number of continuous health monitoring devices are being developed, currently-available solutions do not fully address the needs of cardiac monitoring. The majority of studies have focused on development of the ambulatory devices, rather than exploring their clinical usefulness. Electrocardiographic (ECG) and blood pressure monitors (BP) are generally considered the most mature noninvasive ambulatory cardiovascular monitoring technologies. For other monitors, convincing evidence of the medical value is still lacking.

Further improvements in miniaturization and artifact reduction will remain critical to improve the wearability, patient acceptance and medical value of existing ambulatory cardiovascular monitors. These attributes are limited by factors including mechanical design constraints associated with the sensing mechanisms, power consumption, data storage, the range of ambulatory conditions encountered, signal processing and computational limitations, and cost. With advances in these areas, it is anticipated that cardiac monitoring devices will further contribute to an improved understanding of cardiovascular disease and treatment. 


\section{Chapter 2: Reduction of ECG Motion Artifacts}

\subsection{Motivation / Problem Statement}

The performance of current electrocardiogram (ECG) monitors can be significantly degraded by motion artifacts, which can cause misdiagnoses, inappropriate treatment decisions, and trigger false alarms (Wiese et al. 2005, Friesen et al. 1990, Smith 1984, Burbank and Webster 1978, Tam and Webster 1977).

These artifacts ${ }^{31}$ arise from physiological sources, namely potentials generated in the epidermis by skin stretching due to patient motion and electromyographic signals $^{32}$, and nonphysiological sources, namely external electromagnetic signals such as the $60 \mathrm{~Hz}$ pickup ${ }^{33}$, offset potentials produced by the electrode ${ }^{34}$, signals produced by the interaction of body fluids and the electrode gel (resulting in lack of electrolytic gel), imperfect lead wire and cable connections (Smith 1984, Wiese et al. 2005). Various types of artifacts have been eliminated by modern design. Interference due to power lines has been greatly reduced by the development of the driven-right-leg circuit (Webster 1984). EMG signals can be electronically filtered from the ECG signal (Smith 1984). Artifact caused by the electrode has been eliminated by the development of the floating silver/silver chloride electrode (Smith 1984, Webster 1984).

\footnotetext{
${ }^{31}$ Signal distortion.

${ }^{32}$ Electrical activity in muscles.

${ }^{33}$ The $60 \mathrm{~Hz}$ pickup results from the radiation of energy from $60 \mathrm{~Hz}$ power lines.

${ }^{34}$ Voltage that is stored by the electrode.
} 
In modern ECG monitoring, especially in ambulatory and stress electrocardiography, motion artifacts ${ }^{35}$ due to skin stretching are the main source of noise in ambulatory and stress electrocardiography utilizing traditional $\mathrm{Ag} / \mathrm{AgCl}$ electrodes (Wiese et al. 2005, Smith 1984, Burbank and Webster 1978, Tam and Webster 1977, Webster 1998, Odman and Oberg 1982, Webster 1984) even with the use of sophisticated algorithmic filtering (Wiese et al. 2005). Skin stretching causes mechanical disturbances of the distribution of charges at the electrode-electrolyte interface, which induces a temporary change in the half-cell potential (Webster 1998). Such artifacts can produce signals that resemble the actual P, QRS, and T time waveforms of the ECG, are of similar frequency, and have a comparable or higher amplitude (Smith 1984, Hamilton and Curley 1997, Tong 2002). Figure 2 shows the ECG signal without artifact and motion artifact corrupted ECG signal.

\footnotetext{
${ }^{35}$ Noise introduced to the signal as a result of motion.
} 

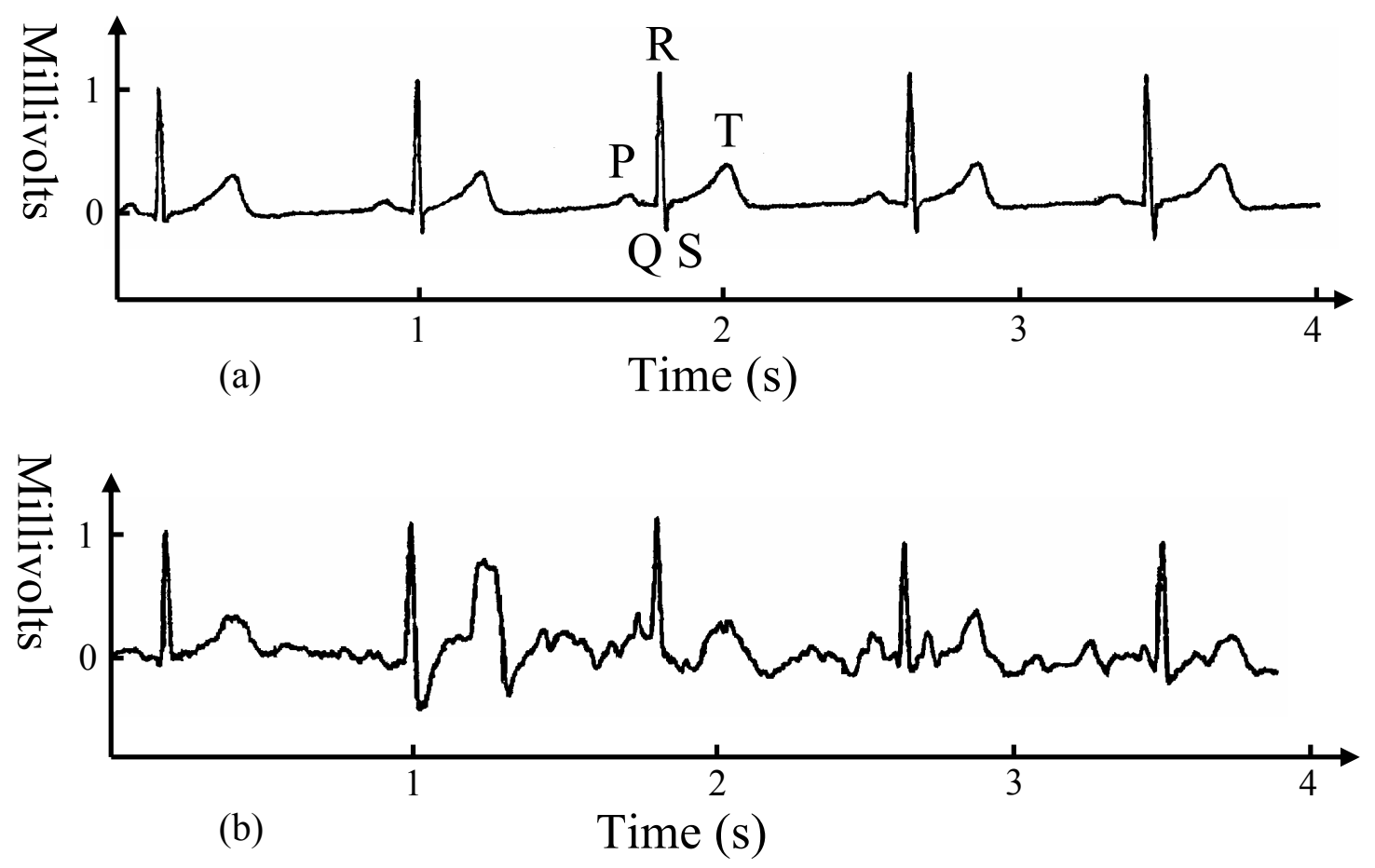

Figure 2. (a) ECG signal without artifact; (b) ECG signal with notion artifact

\subsection{Previous Studies}

The interpretation of the clinical ECG relies on physician or computerized analysis to recognize artifacts (Chia et al. 1991, Kadish 2001). Approaches previously investigated to reduce motion artifacts include skin abrasion at the electrode site (Tam and Webster 1977, Burbank and Webster 1978), and adaptive filtering techniques that utilize as a reference signal either electrode motion measured using single or dual axis accelerometers (Raya and Sison 2002, Tong et al. 2002, Tong 2005) or a two-axis magnetoresistive sensor (Tong et al. 2002), skin stretch related signals measured using a strain gauge (Hamilton and Curley 1997, Hamilton et al. 2000a, 2000b, 1999), or electrode/skin impedance measurements (Hamilton et al. 2000b, Devlin et al. 1984), or voltage difference from two adjacent electrodes 
(Luo and Tompkins 1995). Such efforts are still in progress and artifact reduction remains a challenge for ambulatory ECG monitoring.

\subsubsection{Skin Stretch - the Main Source of ECG Motion Artifact}

Tam and Webster's (1977) research indicated that the change in skin potential is the major source of motion artifact and proposed the method of skin abrasion to minimize this artifact. Odman and Oberg (1982) studied movement-induced potentials in various electrode configurations and concluded that potentials generated by skin deformation beneath the electrode dominate the disturbance pattern in ECG recording. The potential created due to gel movements at the electrode disc, or caused by gel movement relative to the skin are not the main reason of motion artifact. The potentials do not depend on impedance variations. Webster (1984) reviewed various types of artifacts in biopotential recording, the sources and the means for minimizing them. He concluded that the major motion artifact in ECG recording arises from the skin and not the electrode. Burbank and Webster (1978) studied the skin stretch-artifact properties and quantified the skin stretch induced artifact in biopotential recording. The amount of artifact can reach up to $17 \mathrm{mV}$ when the skin strain is $6 \%$.

Skin can be considered as a current generator. It actively generates a potential difference between the inside and the outside of the skin. Consequently, the outside of the skin is more negative than the inside. Stretching the skin causes a reduction in the magnitude of this skin potential. Variation of this potential is a source of artifact in electrocardiography. There is no widely accepted interpretation of the variation with skin stretch on the causes of skin potential. The skin potential between the inside and 
outside of the barrier layer is typically $30 \mathrm{mV}$. When the skin stretches, the skin potential decreases to about $25 \mathrm{mV}$ and this $5 \mathrm{mV}$ change is the observed motion artifact (Webster 1984).

Figure 3 shows that the skin consists of three layers: the epidermis, the dermis and the subcutaneous layer. The epidermis is made up of another three layers: stratum corneum, stratum granulosum and the stratum germinativum. The surface layer, stratum corneum, is a layer consist of dead cells, with a thickness between $0.02 \mathrm{~mm}$ and $0.7 \mathrm{~mm}$ (Tam and Webster 1977). New cells form within the germinating layer (stratum germinativum) and over about 30 days move outward through the barrier layer (stratum granulosum) to the stratum corneum on the surface, and then fall of (Webster 1984).

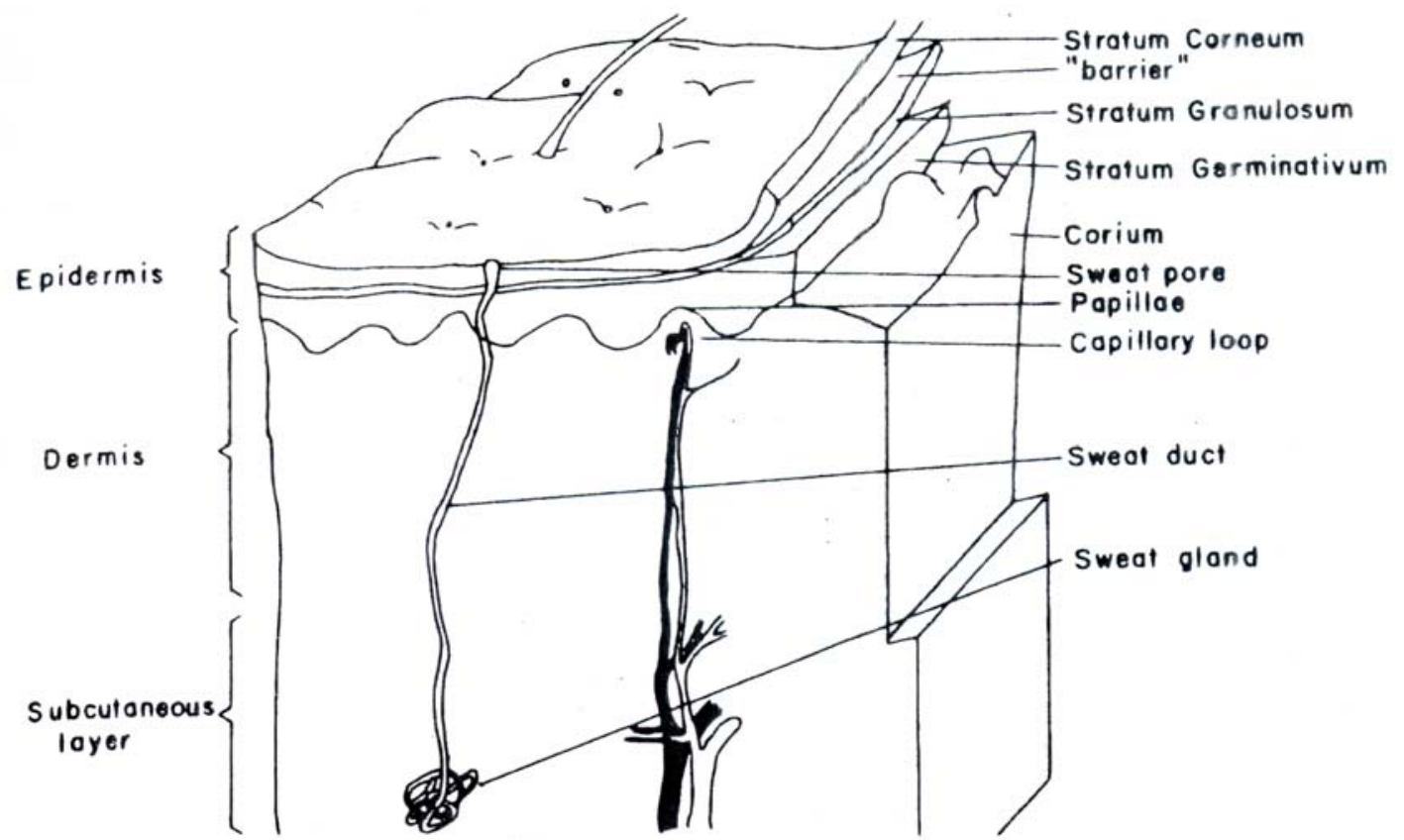

Figure 3. Schematic diagram of the skin (Tam and Webster 1977) 
Thakor and Webster (1978) and de Talhouet and Webster (1996) approximated the skin layers as an equivalent circuit model consisting of resistors and a current supply (Figure 4). Thakor and Webster (1978) hypothesized that the skin potential arises from a constant current source called 'injury current' flowing through the extracellular resistance. In this current model of epidermis, the injury current, caused by difference of metabolic activity between the dead cells of the stratum corneum and the viable cells of the inner layers of the skin, flows through the extracellular channels which cause the negative dc voltage drop from the inside to the outside of the skin. de Talhouet and Webster (1996) validated Thakor's model through experiments, and quantified the change in epidermal skin impedance and skin potential measured using an $\mathrm{Ag} / \mathrm{AgCl}$ electrode with the stretched force that was imposed with hung weights. It was found that the skin potential change and skin impedance change increase with weight in a logarithmic relation (de Talhouet and Webster (1996). The decrease of impedance of the transitional region shunted by the current was attributed to the extracellular channels increase in diameter when stretching the skin (Thakor and Webster 1978, de Talhouet and Webster 1996). 


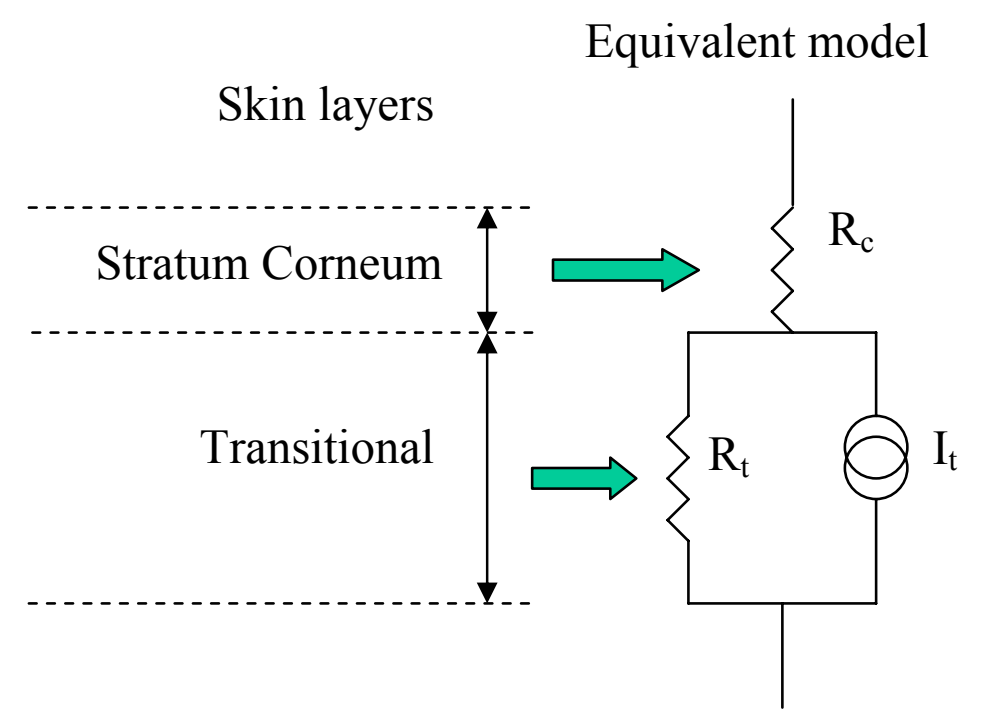

Figure 4. Thakor and Webster's (1978) injury current model of epidermis

\subsubsection{Reduce Motion Artifact by Skin Abrasion and Puncturing}

Tam and Webster (1977) showed that about 20 strokes of fine sandpaper reduce the skin potential artifact from $5 \mathrm{mV}$ to a negligible value. The fine sandpaper strokes scratch through the barrier layer and short circuit the skin potential. However, this method can cause skin irritation and a mild gel must be used to avoid irritation. In addition, they found that the skin regrows in 24 hours and artifact returns.

Burbank and Webster (1978) devised a needle-puncture method to reduce artifact from $5-10 \mathrm{mV}$ to less than $0.2 \mathrm{mV}$ through $100.5-\mathrm{mm}$ skin punctures. A blood lancet protruding $0.5 \mathrm{~mm}$ from an X-Acto knife blade chuck punctured the skin 20 times. The results showed that motion artifact can be minimized by this method, but skin reddening and occasionally small blood droplets can be observed. 
These approaches will cause patient discomfort, prolong procedure preparation time or even infection. Furthermore, they do not provide an effective solution to long term monitoring.

\subsubsection{Reduce Motion Artifact by Adaptive Filtering}

Adaptive filtering has been used in many biomedical applications. It has been applied in fetal ECG recording, cancellation of cardiogenic interference from an impedance plethysmographic signal, $60 \mathrm{~Hz}$ powerline interference cancellation, muscle noise reduction, baseline wander reduction in ECG, and ECG motion artifact reduction (Thakor and Zhu 1991). Other proposed adaptive algorithms to reduce ECG motion artifact include using adaptive neural networks (Xue et al. 1992) and fuzzy-rule-based adaptive nonlinear filters (Kiryu et al. 1994) to track the changing characteristics of the noise in an ECG and adjust the filters to reduce as much the motion artifact as they can.

Figure 5 shows the basic structure of applying adaptive filtering in ECG motion artifact removal. Noise is subtracted from a received signal in an adaptive manner. There are two signal inputs. A primary input contains the ECG signal corrupted by additive and uncorrelated noise. An auxiliary input derived from a second sensor located in the noise field supplies signal that highly correlated with the unknown noise but uncorrelated with pure ECG signal. The adaptive filter estimated noise in an adaptive manner and eliminate the additive noise through the noisy ECG to extract the desired ECG signal. 


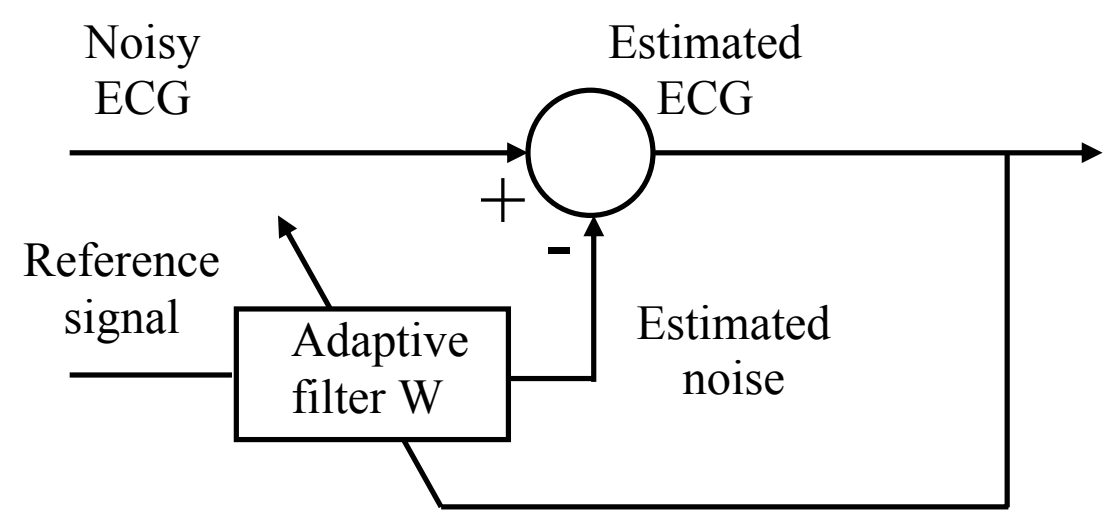

Figure 5. Adaptive filter structure

Tong et al. $(2002,2005)$ monitored the electrode acceleration by attaching a 3$\mathrm{D}$ accelerometer and a 2-axis anisotrophic magnetoresistive sensor respectively on the exposed surface of an electrode (Figure 6). The electrode acceleration signal was measured simultaneously with ECG signal and used to estimate motion artifact with adaptive filtering. Motion artifact was induced by pushing on the electrode, pushing on the skin around the electrode and pulling on the electrode lead wire. The results indicated that adaptive filter with a reference motion sensor can significantly educe motion artifact. The improvement ranges from $24 \%$ to $91 \%$ depending on the motion type and the reference sensor type. However, imposed electrode motion may not fully replicate in daily activities, and skin stretch caused by daily activities were not taken into account. 


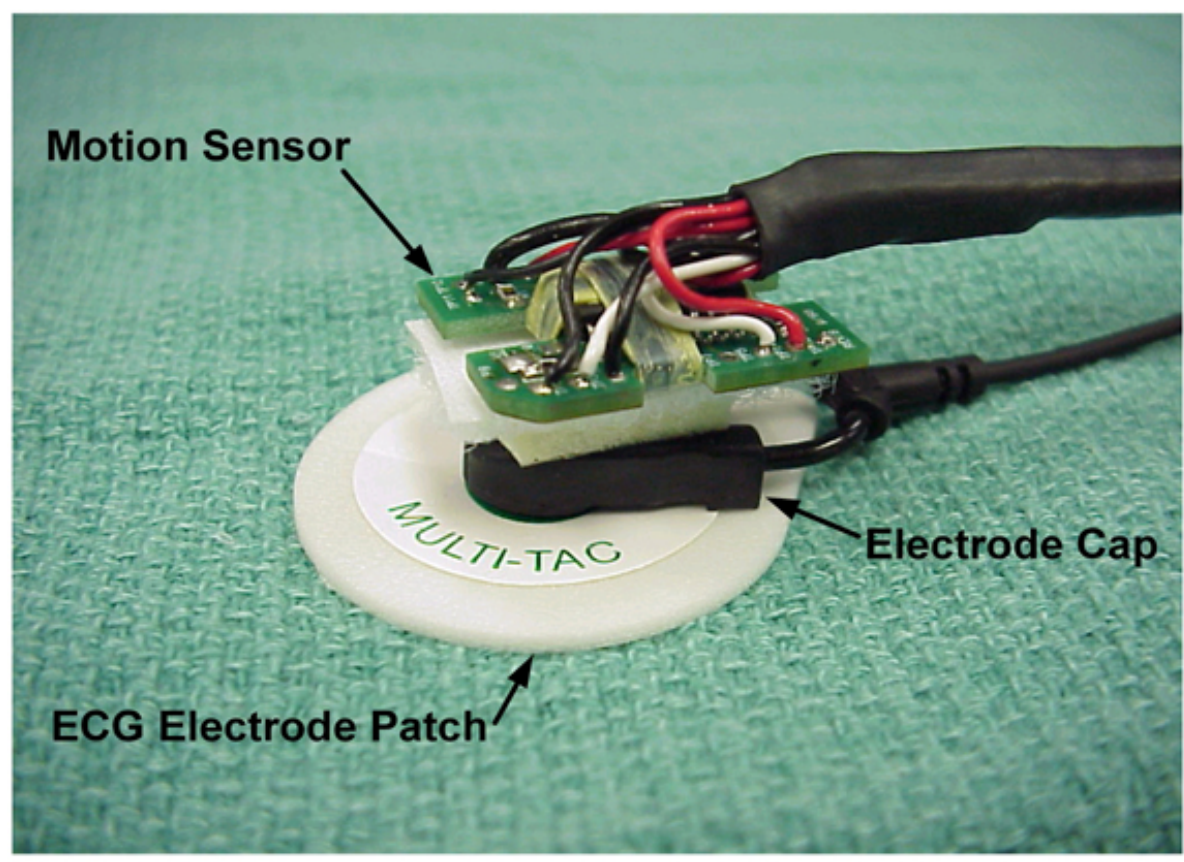

Figure 6. Measurement of electrode motion using an accelerometer (Tong 2000)

Raya and Sison (2002) attached on the subject's back a 1-D and 2-D accelerometer respectively to measure body acceleration as the reference signal to reduce ECG motion artifact. The subject was walking and running during the stress ECG test. The results showed that motion artifact in stress ECG was reduced effectively by using a single-axis acceleration signal as the noise reference. A single noise reference gave a better result than dual-axis noise reference regardless of the adaptive filtering algorithm applied. Recursive least square (RLS) algorithm gave a superior performance than Lease Mean Square (LMS) algorithm. Since RLS converges faster than LMS, it can improve the signal to noise ratio in a rapidly varying environment compared with LMS. The noise reduction results were not quantified. Local skin deformation under the electrode were not taken into account. 
Hamilton et al. (Hamilton and Curley 1997, Hamilton et al. 2000a, 2000b, 1999) mounted a strain gauge and an optical bend sensor on the exposed surface of an electrode respectively to measure electrode deformation as the noise reference. Artifact was generated by pulling on the skin and pushing on the electrode. The result indicated that adaptive filter can effectively reduce motion artifact. Different adaptive filtering algorithms have different noise reduction performance. Filter order larger than four don't contribute greatly to further noise reduction. The average noise reduction in false detection for the controlled conditions (pulling on the skin and pushing on the electrode) is $72.6 \%$. The residual artifact may result from nonlinear behavior of the foam-sensor arrangement used to indicate skin stretch or nonlinear relationship between the skin stretch and the artifact amplitude. In addition, during ambulatory monitoring significant artifact may be generated by skin stretch that does not deform the electrode in ways that are well detected by the strain gauge or the optical bending sensor. Imposed electrode bending may not fully replicate in daily activities and skin stretch caused by daily activities was not taken into account.

Devlin et al. (1984) used electrode / skin impedance variations to estimate and reduce the relative amount of electrode motion artifact in the ECG signal, so as to reduce false positive alarms triggered by motion artifact. The time-varying impedance of the electrode / skin interface was monitored by passing a small AC current through the ECG electrode and measuring the resultant potential difference across the electrodes. Artifacts were introduced by scratching and tugging on the electrodes, and abrupt physical movements. The results showed that $82 \%$ of the false positive alarms can be eliminated, with a loss of $7 \%$ of the true positives. However, 
the torso impedance will introduce the possibility of impedance artifact generated by breathing or heart pumping. Hamilton et al. (2000b) further investigated using skin / electrode impedance to adaptively filter motion artifact from ECG signals. Low frequency impedance was measured in Hamilton's (2000b) study, different from the $20 \mathrm{kHz}$ impedance used by Devlin et al. (1984), since low frequency impedance should be more sensitive to changes in skin impedance and less sensitive to changes in torso impedance. The results showed that artifact reduction decreased with increasing impedance frequency. Artifact reduction improved with the order of the filter, but the improvement was not significant for filter orders larger than four. However, it was unclear that how the movement-induced artifact is correlated with Luo and Tompkins (1995) obtained voltage difference from two adjacent electrodes ( $5 \mathrm{~mm}$ apart) as a noise reference signal. The artifacts were induced by raising the right lower arm from a relaxed position until the lower arm makes contact with the brachium. The results showed that RLS algorithm converges faster than the LMS algorithm and performs satisfactorily in reducing ever rapidly varying brachial artifact. The method also significantly improved the low frequency baseline shift. Noise reduction results were not quantified in the study. Local skin stretch caused artifact was not taken into account in this method.

These studies demonstrated that adaptive filtering is an effective method to reduce motion artifact in ambulatory ECG, stress test and long term ECG monitoring. However, the estimated noise reduction could vary significantly depending upon the source of skin stretch, namely applied electrode motion or deformation, or body motion, etc. None of the above method used the skin strain as the auxiliary input to 
adaptive filtering. No single combination of reference variable, sensor, and filtering algorithm could reduce the noise in a reproducible/consistent manner for all electrode or body motions studied. The results suggests that an important contributing factor for the lack of consistency in the estimated noise reduction is that the references variables used, namely electrode acceleration, electrode strain etc., were not adequately correlated with the induced motion artifact. In addition, the induced motion artifact in previous studies does not take into account skin stretch caused by daily activities. As a result, motion artifact generated by skin stretch that does not deform the electrode or is not caused by electrode motion etc. will not be detected by previously used sensors as an auxiliary input to adaptive filtering.

\subsection{Research Objective}

The research objective is to reduce skin stretch induced artifacts in the measured ECG. To achieve this, a method and prototype was developed to directly measure skin stretch simultaneously with ECG. This process involves the integration of an optical technique in an ECG electrode. Using the measured skin stretch data, skin stretch induced artifacts are reduced in the measured ECG with adaptive filtering technique.

Different from previous studies, in this work skin stretch is directly measured as an auxiliary input in adaptive filtering. Figure 7 shows the block diagram of the cause and effect relationship between variables and motion artifact in previous approaches and this study. 


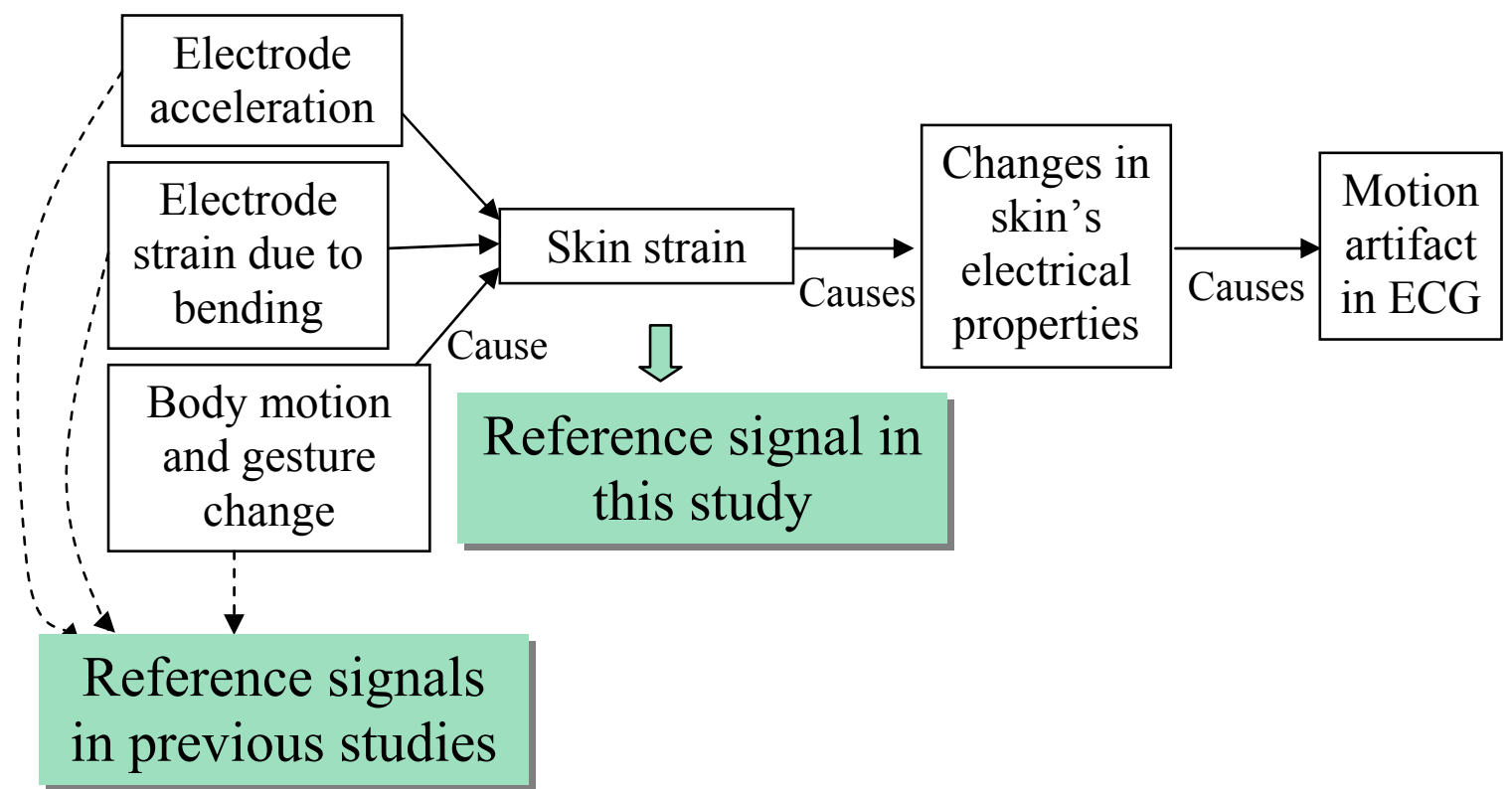

Figure 7. Block diagram of the cause and effect relationship between variables and motion artifact

\subsection{Research Methodology}

Adaptive filtering method is used to transform the optical sensor output into the motion artifact induced in the ECG leads. An estimate of the artifact is then subtracted from the corrupted signal resulting in an electronic signal with reduced motion artifacts. Figure 8 shows the electrode/ sensor system and adaptive filter structure to reduce ECG motion artifact. 


\section{Electrode}

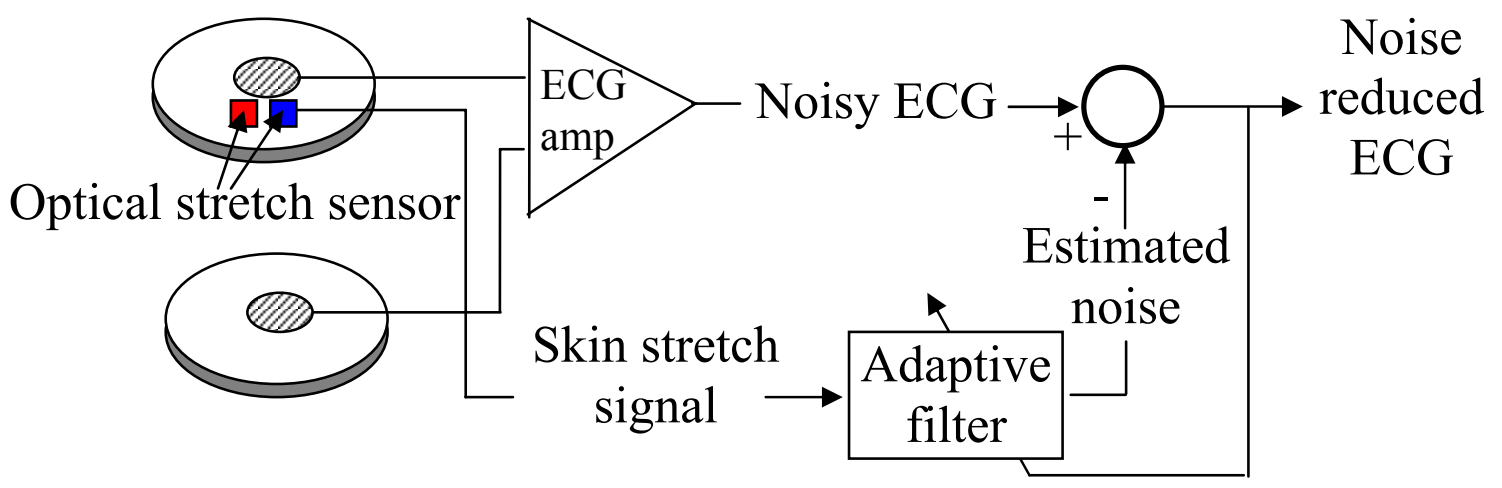

Figure 8. Electrode/ sensor system and adaptive filter structure to reduce ECG motion artifact

The general research methodology includes the following procedures:

1) Incorporate a noninvasive optical technique to measure skin strain in the ECG electrode package

2) Calibrate the optical strain sensor in-vitro and in-vivo in terms of sensitivity and measurement range

3) Record ECG with induced artifacts and skin strain simultaneously, and reduce the artifacts offline with adaptive filtering

4) Quantify artifact reduction performance of this sensor system and method

5) Test performance of this sensor system and method under various types of motions that mimic everyday activities 


\section{Chapter 3: Optical Sensor Calibration and Prototyping}

\subsection{Integration of an Optical Sensor into an ECG Electrode}

Figure 9 illustrates one embodiment of a skin-mounted physiological recording electrode assembly, with the isometric view on the left and exploded isometric view on the right. The electrode assembly has a foam pad with the font surface contacting the skin and a rear surface connecting to the lead wires. The front surface of the foam pad has a central recessed region filled with liquid gel or solid gel to establish electrical contact between the electrode and the skin. The $\mathrm{Ag} / \mathrm{AgCl}$ electrode resides in the central recess of the foam pad with a connector end protruding from the foam pad. A clip grips the connector end of the electrode and connects a lead wire to recording and processing equipments. An optical sensor and a light emitting diode (LED) are embedded in the foam pad near the electrode site, with a recessed region underneath the sensor and the LED (Liu and Pecht 2006, Pecht and Liu 2005). 


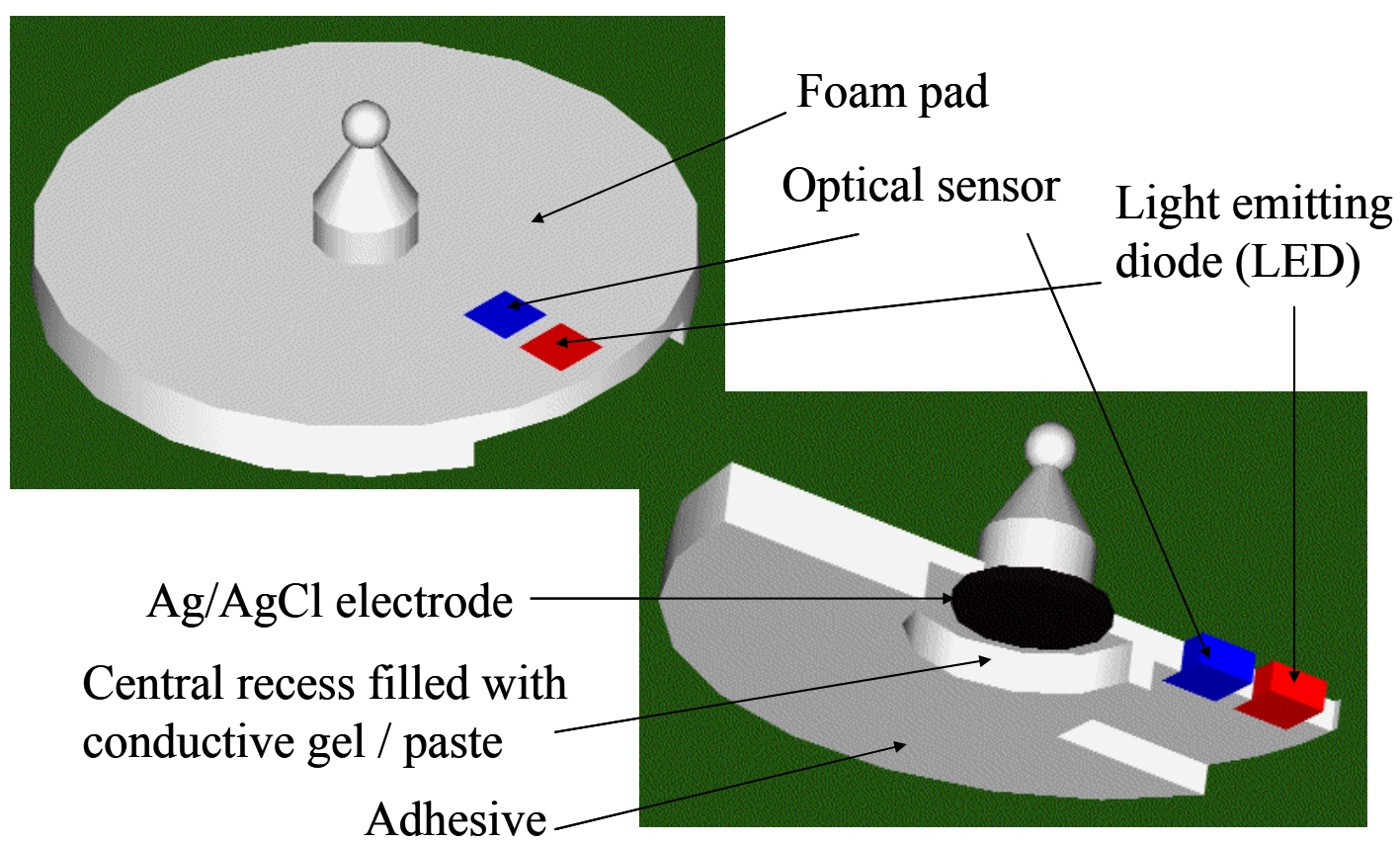

Figure 9. Isometric view (left) and exploded isometric view (right) of an optical sensor integrated electrode

Optical components integrated in an electrode are illustrated in Figure 10. LED illuminates the skin surface, revealing surface details (slight imperfections) that the optical sensor can use to track movement. The optical sensor consists of a CMOS image sensor and a digital signal processor to determine the direction and amount of the relative movement between the sensor and the imaging area beneath it. Lenses are integrated with the optical sensor and LED to focus the light and image. 


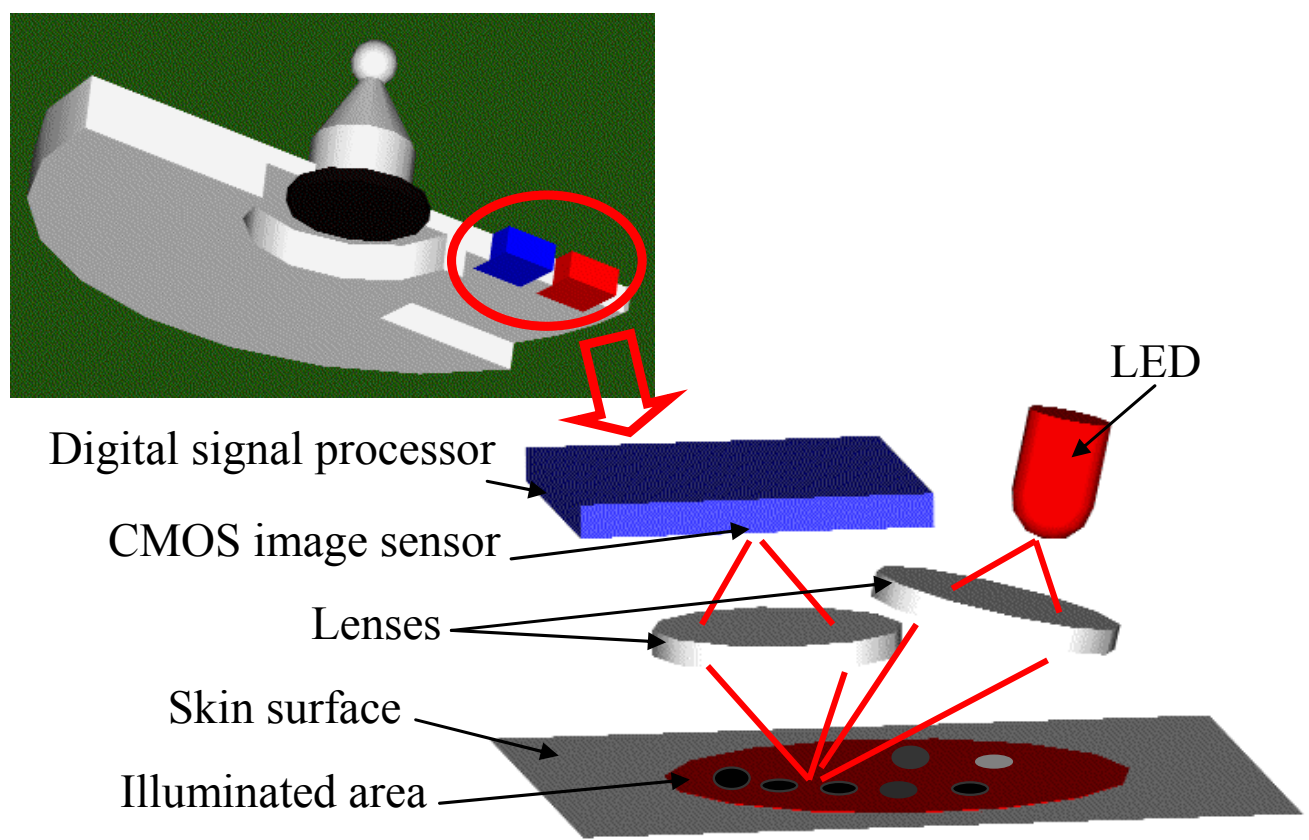

Figure 10. Optical components integrated in an electrode

Optical sensors used in this study are based on optical navigation technology. Optical navigation has also been used in target tracking in military applications, optical mouse, and motion compensation in hand-held video cameras, which is critical to the practical utility.

Figure 11 illustrates the illuminated skin surface area underneath the CMOS image sensor. The CMOS sensor is an image array ranges from $16 \times 16$ pixels to 30 $\times 30$ pixels. Let $\mathrm{L}$ be the distance between the fixed edge and the imaging area. Assuming the skin underneath the electrode and the optical sensor has a uniform strain field, skin strain is calculated as $\Delta \mathrm{L} / \mathrm{L} . \Delta \mathrm{L}$ is proportional to the optical sensor output. When the skin under the electrode is stretched, the sensor detects relative movement of the area underneath it due to skin strain of the adjacent area. The sensor identifies common features in sequential images and calculates the feature 
displacement in DSP. In this manner, the skin strain is proportional to the sensor output.
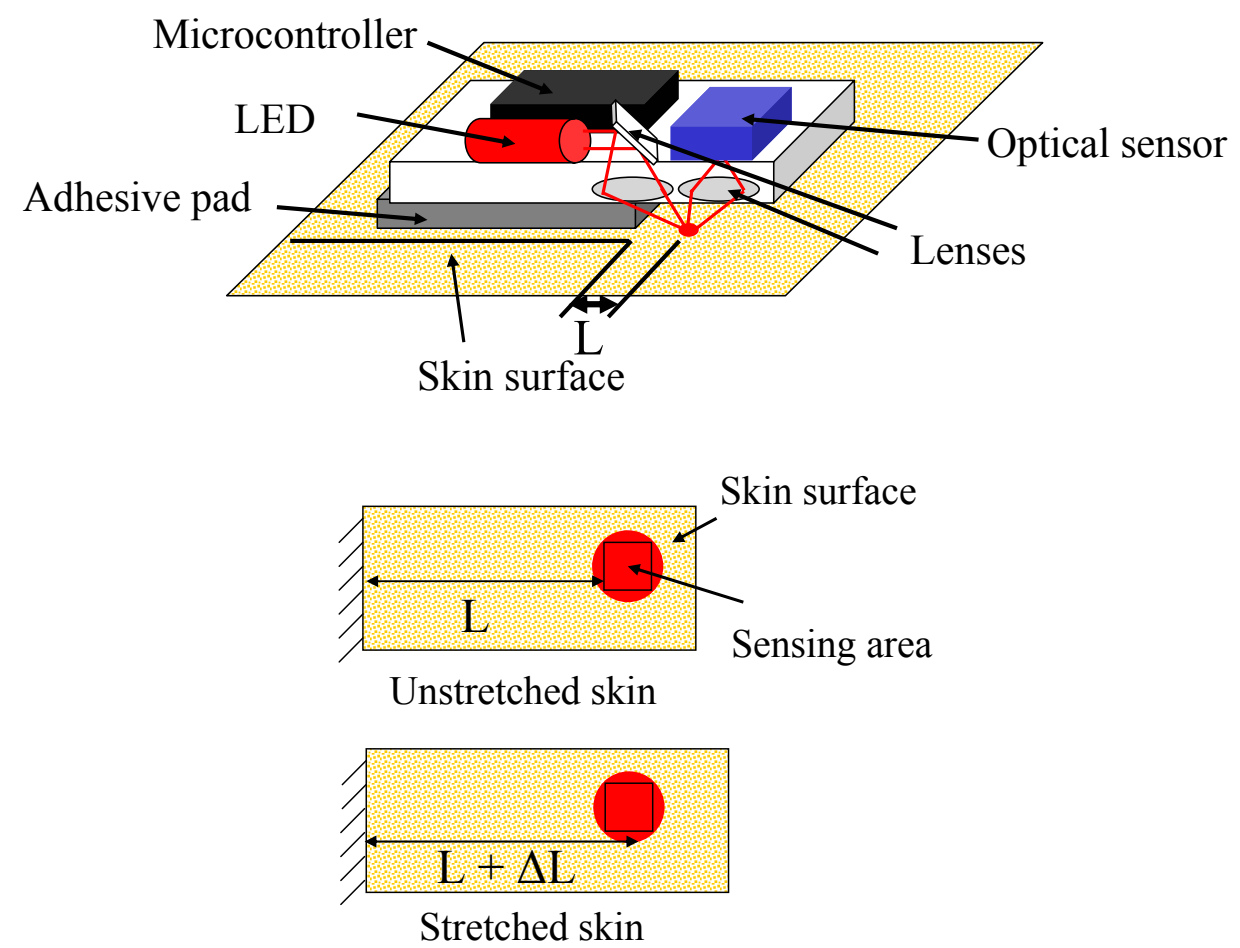

Figure 11. Illuminated skin surface area underneath the CMOS image sensor

Figure 12 shows two images (Avago Technologies 2005) with image (b) take a short time after image (a), while there is relative movement between the sensor and the area beneath it. The optical sensor integrated with a digital signal processor identified common features in sequential images to determine the direction and amount of relative displacement of the area underneath the sensor. 


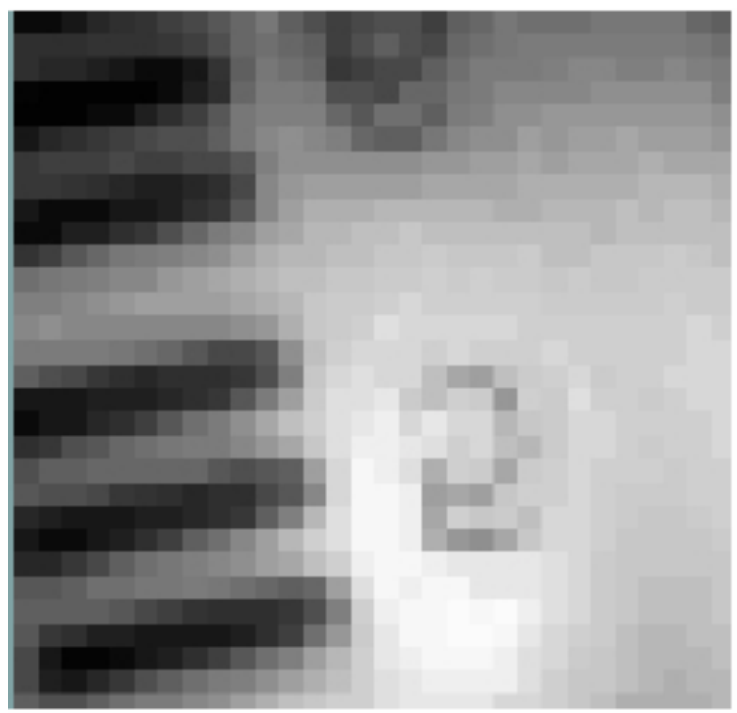

(a)

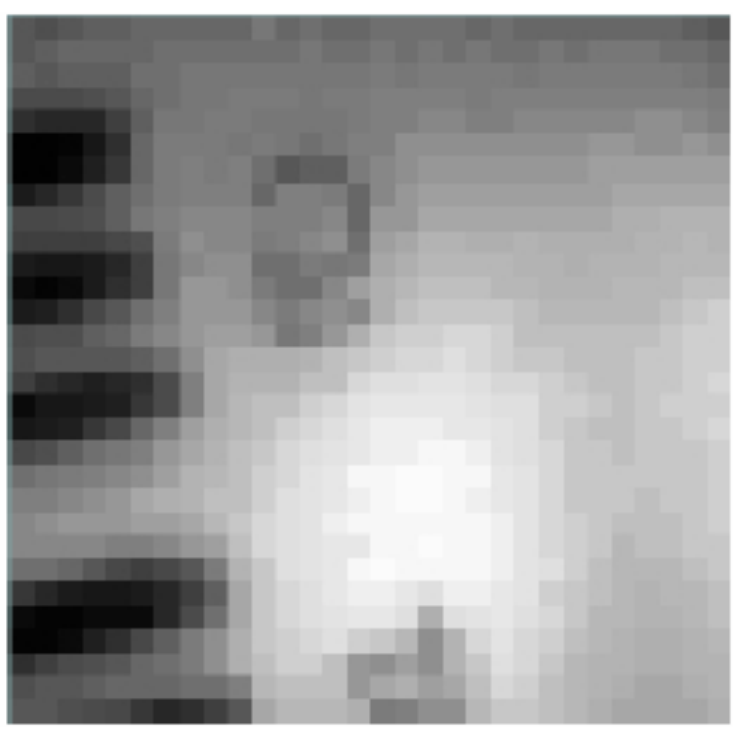

(b)

Figure 12. The optical sensor identified common features in sequential images to determine the direction and amount of relative displacement of the area underneath the sensor. Image (b) was take a short time after image (a), while there is relative movement between the sensor and the area beneath it (Avago Technologies 2005).

\subsection{Calibration of the Optical Skin Stretch Sensor}

To demonstrate the feasibility of using the optical sensor to measure soft material strain, experiments were carried out to calibrate the optical strain sensor performance in vitro. An animal skin specimen was clamped and stretched in a controlled condition. The experiment is illustrated in (b)

Figure 13. The optical sensor is an image sensor with a resolution 0.03175 $\mathrm{mm}^{36}$. The optical sensor is attached and fixed at one end of the specimen. This is to ensure that there is no relative movement between the sensor and one edge of the fabric specimen. The sensor is connected to a computer by a universal serial bus

\footnotetext{
${ }^{36}$ The optical sensor's resolution is its ability to detect spatial differences. In this study the sensor is capable of distinguishing 800 distinct dots in a line 1 inch long, so its resolution is $0.03175 \mathrm{~mm}$.
} 
(USB port). The initial distance between the two inner sides of the clamp is recorded using a micrometer caliper. The specimen is stretched step by step with the amount of elongation measured by a micrometer caliper. The relationship of the strain calculated from the elongation amount and the sensor output is plotted in the computer to demonstrate the feasibility of using the optical sensor to measure soft material strain.

Based on optical navigation technology, the sensor optically measures changes in position by sequentially acquiring 1500 surface images per second and mathematically determining the direction and magnitude of movement. The process overlays two sequential images and slides them around to a number of positions, until the best fit is found. The image capturing rate is fast enough so that sequential pictures overlap. The higher the image capturing rate, the higher the relative movement speed it can respond to (the maximum speed the optical sensor can respond to is proportional to the image capturing rate). For example, if the dimension of the optical sensor is $0.8 \mathrm{~mm} * 0.8 \mathrm{~mm}$, and the magnification factor is 1 , the maximum speed the optical sensor can capture is $0.8 \mathrm{~mm} /(1 / 1500 \mathrm{~s})=1200 \mathrm{~mm} / \mathrm{s}$. The sensor used in this study can detect up to $355.6 \mathrm{~mm} / \mathrm{s}$, while the typical skin elongation speed is less than $100 \mathrm{~mm} / \mathrm{s}$. 


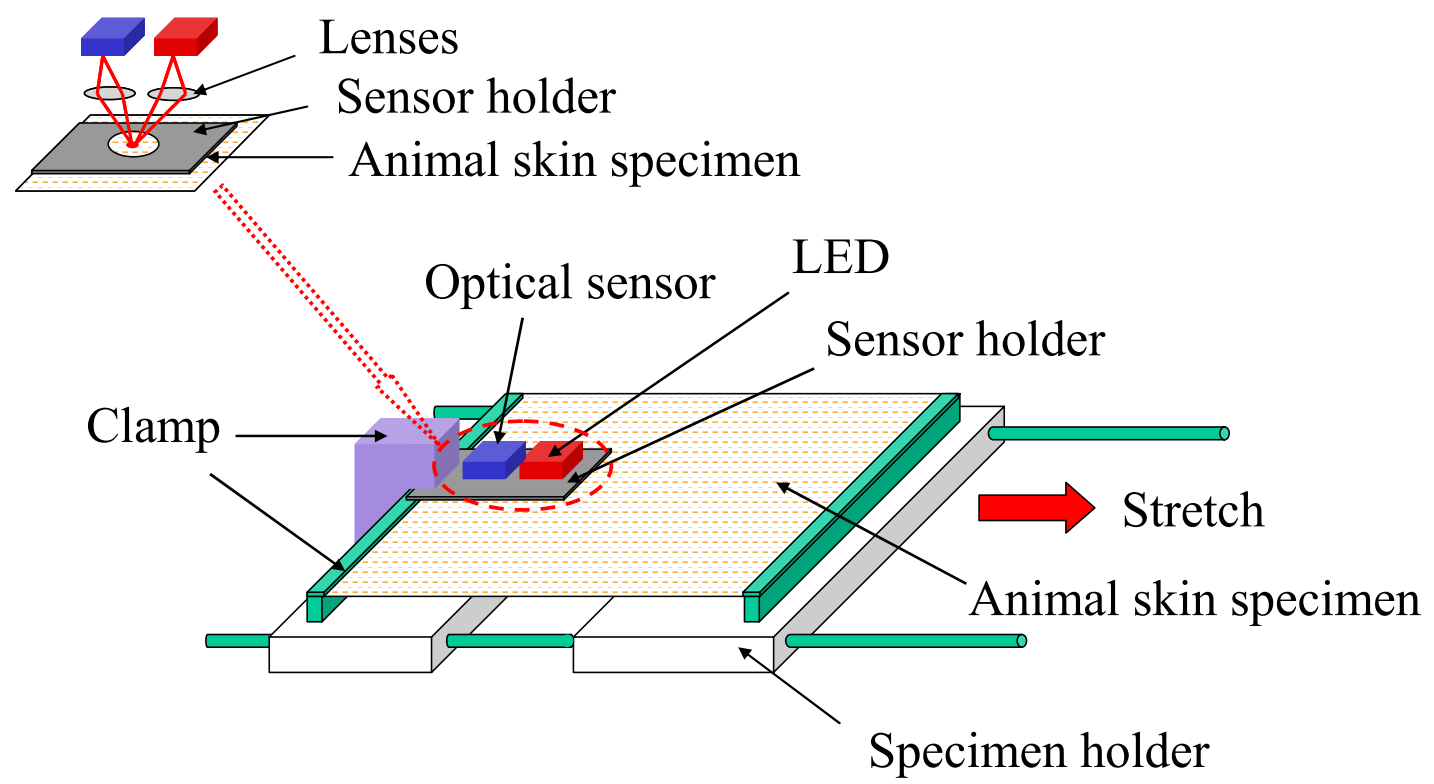

(a)

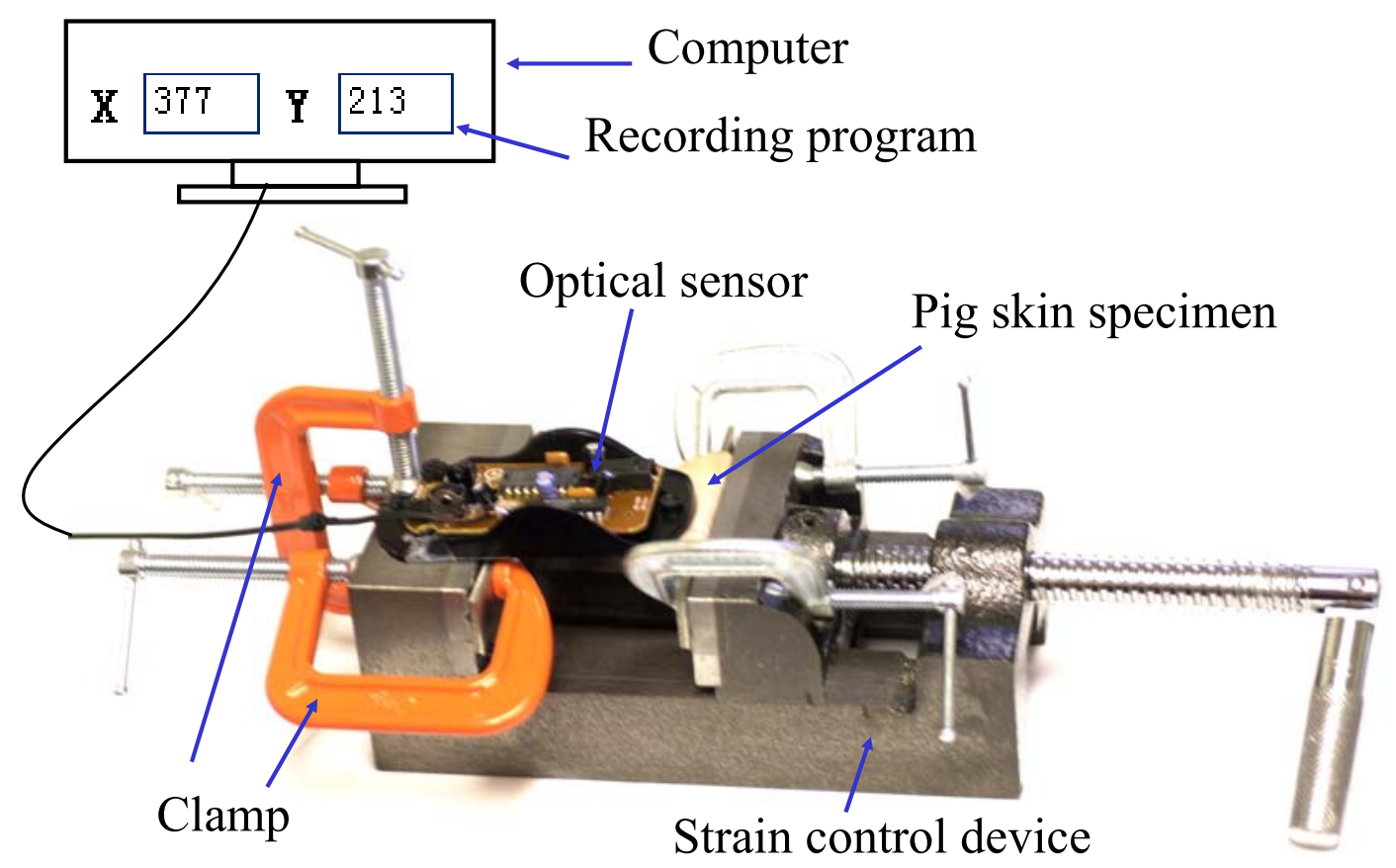

(b)

Figure 13. In-vitro Calibration of the optical stretch sensor

The sensor's output position was recorded by a Delphi program. The program can run on MS Windows and record the sensor output by simply pressing the blank 
key to store the data, as shown in Figure 14. Let $x$ and $y$ be the sensor output - offset values in $x$ and $y$ directions. Let $x_{0}$ and $y_{0}$ be the initial sensor output. Assuming the strain is one dimensional, the optical sensor output $S$ is defined as

$$
S=\sqrt{\left(x-x_{0}\right)^{2}+\left(y-y_{0}\right)^{2}}
$$

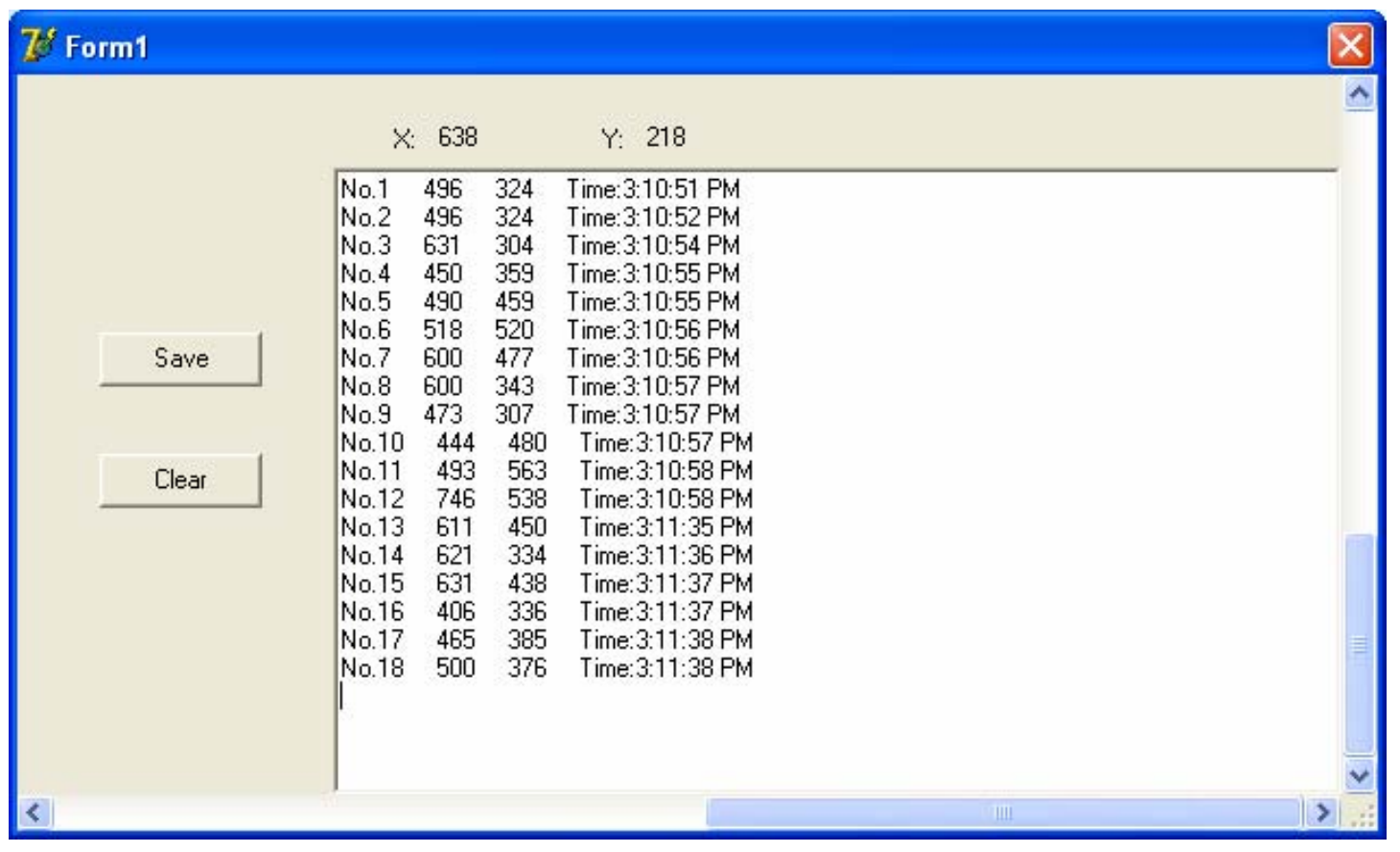

Figure 14. Cursor recording program

Monoaxial tensile experiment was performed to calibrate the optical stretch sensor invivo, as described in (a) 
(b)

Figure 15. Static deformation was maintained during the experiment. Two legs of a micrometer were attached to a forearm by adhesive tape. The two legs were driven apart so as to exert tensile stress on the skin and extend the skin between the two legs. One edge of the optical sensor was attached to the skin surface using double-sided adhesive tape, while the other end hanging above the skin. The micrometer was used to stretch the skin and to measure the distance between the two legs. The optical sensor output was recorded in the same computer program as described in the in vitro calibration experiments.

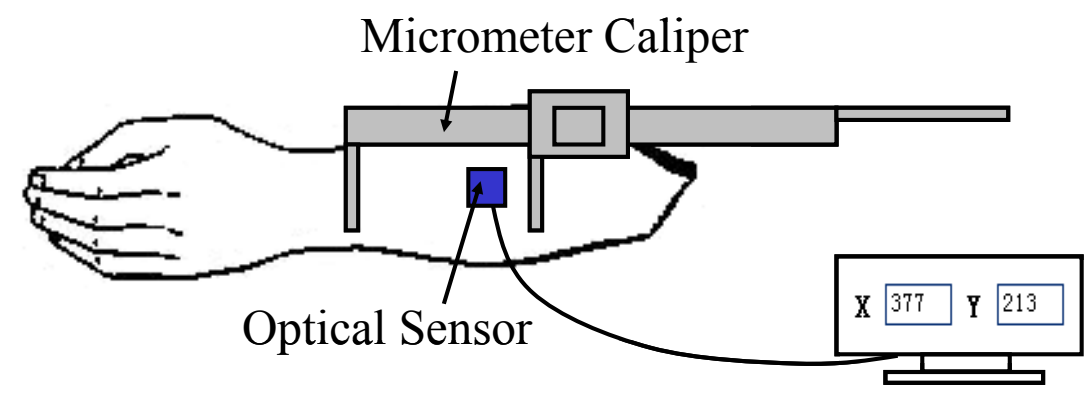

(a) 


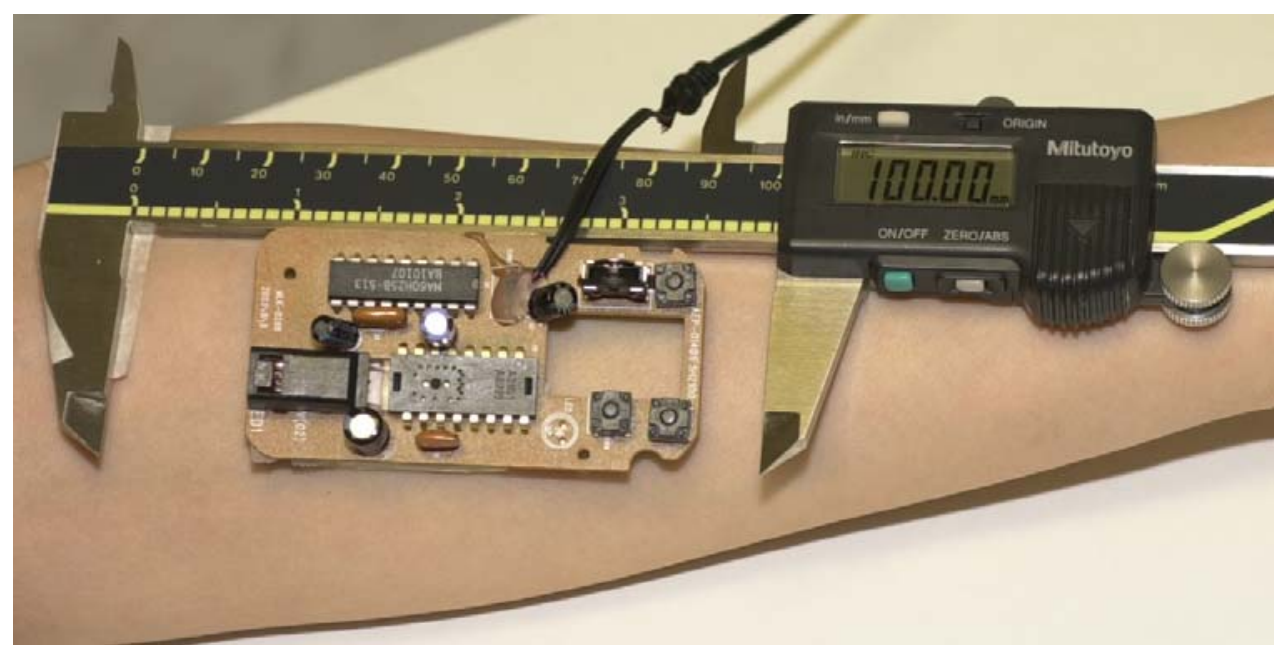

(b)

Figure 15. In-vivo calibration

The transfer function of the optical sensor establishes dependence between the optical sensor output $S$ and skin strain $s$. A transfer function of a sensor can be any form. In this study, the in vitro and in vivo calibration results, as shown in Figure 16 and Figure 17 respectively, demonstrated that a linear relationship was achieved between the sensor output and the amount of skin strain calculated from the elongation. The results demonstrate that the optical sensor can be used as a soft material strain sensor. The transfer function of the optical sensor can be written as

$$
S=b s
$$

where $s$ is the skin strain calculated as the amount of elongation of the skin divided by the initial length, and $b$ is the sensor sensitivity. 


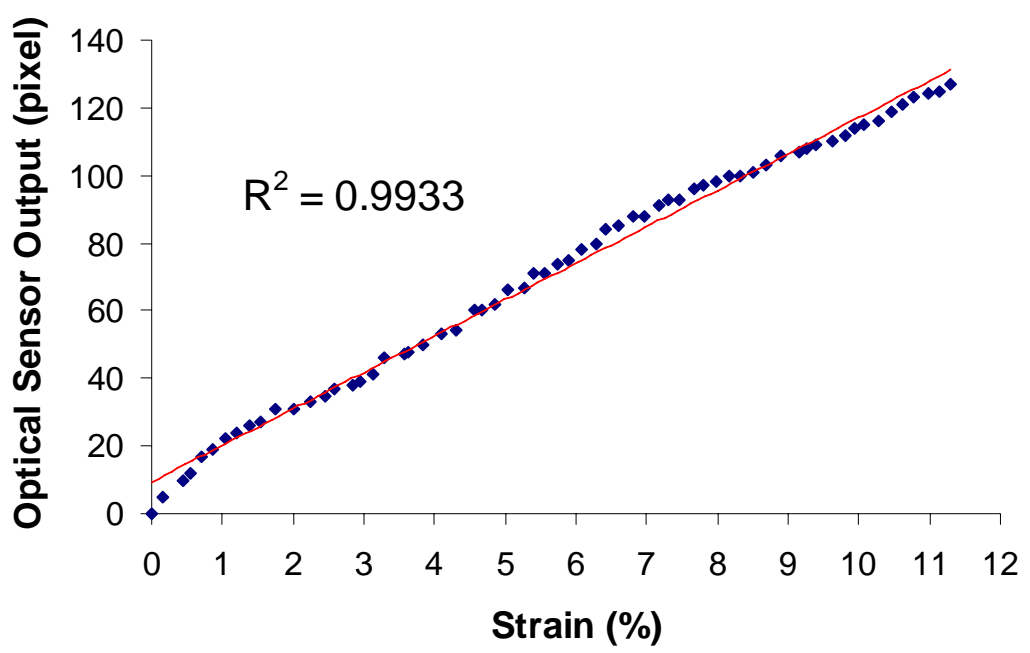

Figure 16. Optical sensor output versus the strain of an animal skin specimen

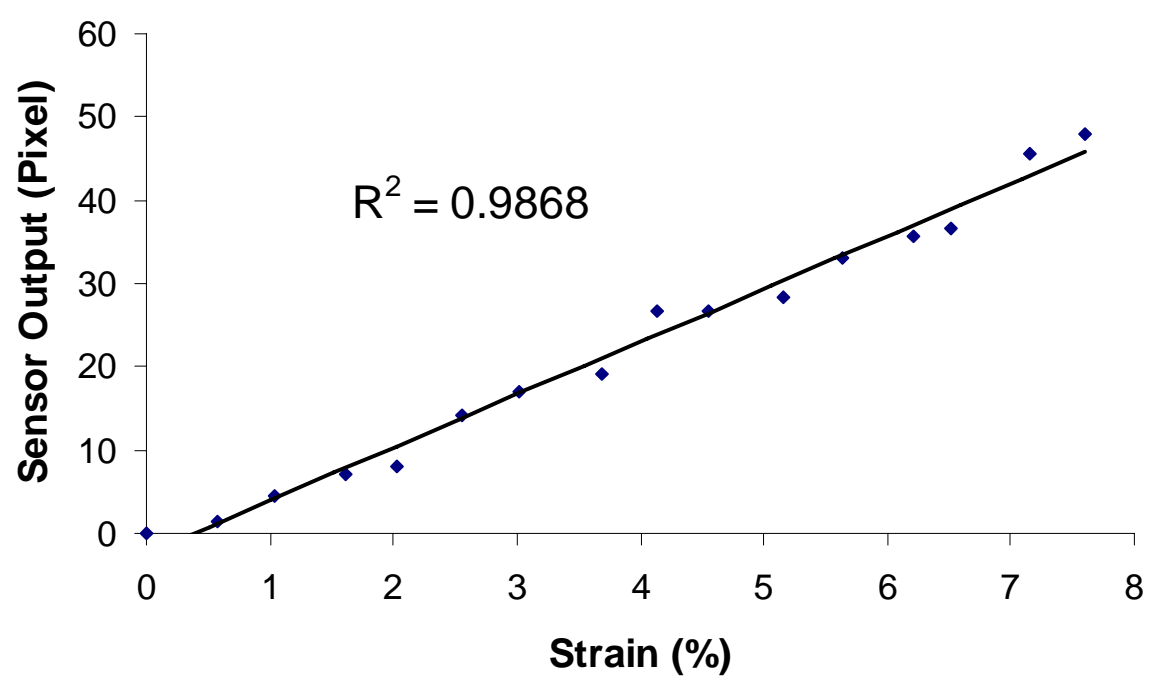

Figure 17. In vivo calibration

\subsection{Prototyping}

The optical strain sensor has been calibrated in vitro and investigated in terms of sensitivity and measurement range. Optical sensor integrated electrode is then prototyped and tested in-vivo. 
The relationship between the optical sensor sensitivity / slope $b$ and the distance between the fixed edge and the imaging field $(\mathrm{mm}) L$ is investigated. The optical sensor output versus the skin strain was measured with a particular $L$ value. The distance $L$ was changed five times from $35.27 \mathrm{~mm}$ to $9.28 \mathrm{~mm}$, and the experiment was repeated for each $L$. The results are shown in Figure 18 and Table 1 . It was obvious that the larger the $L$, the larger the sensitivity for measuring skin strain. The relationship between the sensitivity and $L$ is shown in Figure 19. The sensor sensitivity is linearly dependant on the distance $L$.

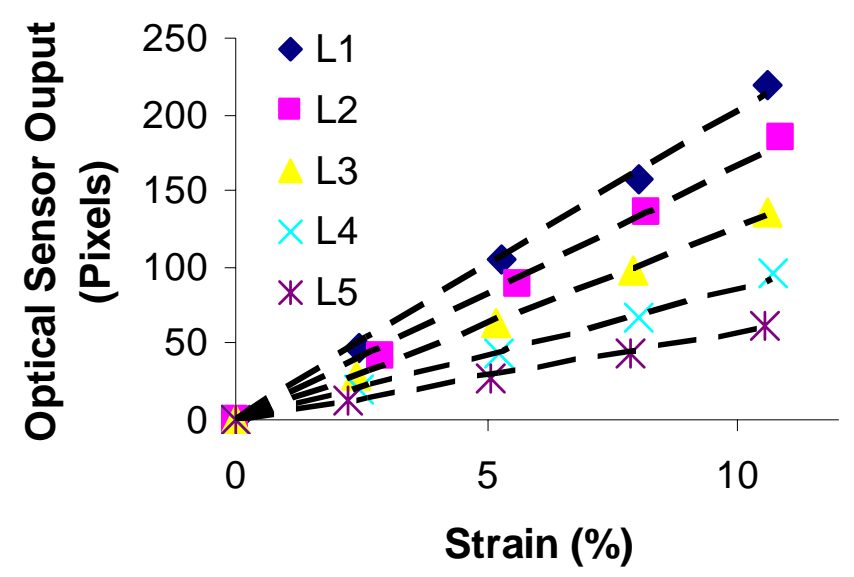

Figure 18. Optical sensor output vs. skin strain with different L (distance between the fixed edge and the imaging field)

Table 1. Optical sensor sensitivity vs. L

\begin{tabular}{|c|c|c|c|}
\hline No. & $\mathrm{L}(\mathrm{mm})$ & Sensitivity $\mathrm{b}$ & $\mathrm{R}^{2}$ \\
\hline 1 & 35.27 & 20.24 & 0.998 \\
\hline 2 & 28.54 & 16.64 & 0.996 \\
\hline 3 & 22.23 & 12.61 & 0.999 \\
\hline 4 & 15.78 & 8.63 & 0.996 \\
\hline 5 & 9.28 & 5.69 & 0.999 \\
\hline
\end{tabular}




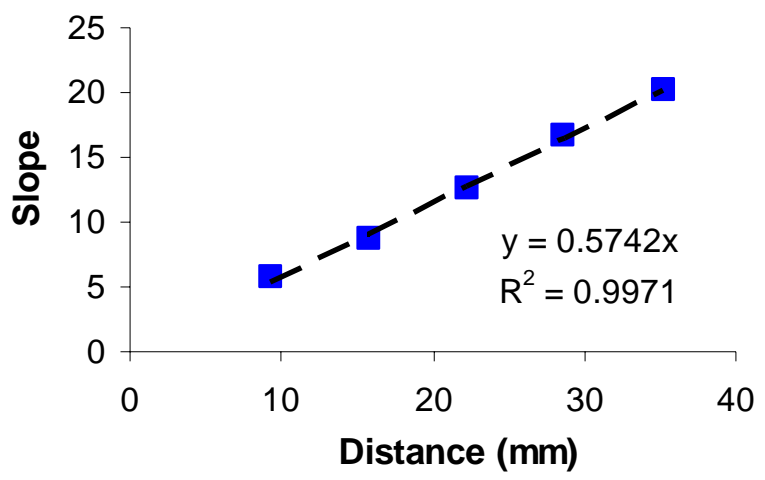

Figure 19. Optical sensor slope / sensitivity vs. distance $L$

Based on the results above, the sensitivity / slope $b$ of the optical sensor can be represented by

$$
b=k L
$$

Where $L$ is the distance between the fixed edge and the imaging field (mm); $k$ is a factor depending on the sensor resolution.

The optical sensor's resolution can affect its sensitivity to measure skin strain. Theoretically, the larger the sensor resolution, the better the sensitivity for measuring skin strain. The sensor's sensitivity to measure skin strain also depends on $L$ (the distance between the fixed edge and the imaging field), as previously discussed. 


\section{Chapter 4: Adaptive Filtering Theory}

\subsection{Introduction}

An adaptive filter is a digital filter that processes a signal by adaptively adjusting itself to the input signal. This ability to operate in an unknown environment to track time variations of input statistics makes adaptive filters a powerful tool in signal processing and control applications. In comparison, a non-adaptive filter has static filter coefficients, which require a priori information about the statistical characteristics of the input data and is not adequate to be applied for nonstationary inputs.

Adaptive filters have been successfully applied in diverse fields including communications, radar, sonar, seismology and biomedical engineering. When applying an adaptive filter, an input vector and a desired response are used to compute an estimate error, which is in turn used to control the values of a set of adjustable filter coefficients.

Adaptive filters have four main classes of applications, including identification, inverse modeling, prediction, and interference or noise cancellation. Detailed description of these four applications can be referred to the book of Haykin (2001). In this thesis work, adaptive filters are used for the purpose of noise cancellation, i.e., to subtract noise from a received signal in an adaptive controlled manner so as to improve the signal-to-noise ratio. 
Adaptive noise canceling was initially applied by Bell Telephone Laboratories in echo cancellation on telephone circuits around 1965, with the speech signal itself utilized in performing the adaptation (Haykin 2001). Widrow et al. (1975) developed the adaptive line enhancer in 1965 to eliminate $60-\mathrm{Hz}$ interference in electrocardiography.

Adaptive noise canceling is a method of estimating signals corrupted by some unknown and additive noise or interference, with the cancellation process being optimized. This scheme operated on the outputs of a primary sensor and a reference sensor. The primary sensor supplies a desired signal of interest buried in noise to the adaptive filter. There are two assumptions here: (1) the signal and noise at the output of the primary sensor are uncorrelated; and (2) the signal at the output of the reference sensor is correlated with the noise component of the primary-sensor output. The noise is adaptively filtered and subtracted from the primary input to obtain the signal estimate. In the adaptive noise canceling system, the system output serves as the error signal for the adaptive process. With a proper algorithm, the filter can operate under changing conditions and readjust itself continuously to minimize the error signal (Thakor and Zhu 1991).

Figure 20 shows the electrode-sensor system and adaptive filter structure to reduce ECG motion artifacts developed in this thesis work. The method uses the corrupted ECG signal as a primary input and skin strain signal as a reference input, which is correlated with the noise in the primary input. The system consists of a pair of electrodes, with an optical sensor on one electrode, an ECG amplifier and a computer. The primary input $d(n)$ is detected by these two electrodes. The skin 
strain signal $u(n)$ is measured from an optical sensor incorporated in an electrode. The adaptive filter estimates noise in an adaptive manner and eliminates the additive noise $y(n)$ through the noisy ECG resulting in an electronic signal $e(n)$ with fewer motion artifacts. The adaptive canceller tends to minimize the mean-square value of the overall output $e(n)$, thereby causing the output be the best estimate of the desired signal in the minimum-mean-square error sense. $e(n)$ is in turn used to adjust the filter coefficients. This is described in detail as follows.

\section{Electrodes}

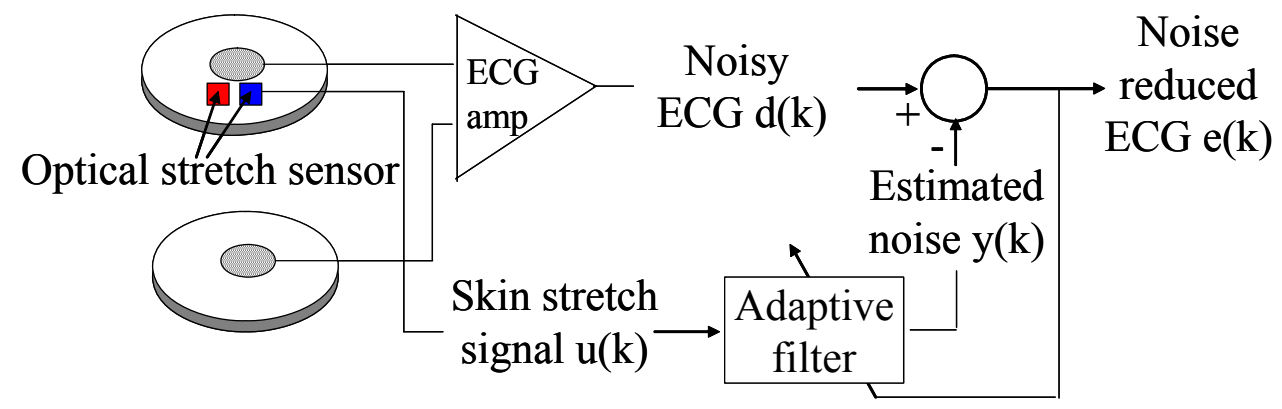

Figure 20. Electrode-sensor system and adaptive filter structure to reduce ECG motion artifacts

Let $s$ be the true ECG signal and $a$ be the artifacts added to the true ECG signal. $d$ is the corrupted ECG signal given by $d=s+a$, and $u$ is the skin strain signal from the optical sensor. $y$ is the estimate of the artifacts. The system output / error $e$ is defined as $e=s+a-y$. The objective of the adaptive filtering is to minimize $a-y$. This can be achieved by minimizing $E\left(e^{2}\right)$ :

$$
\begin{aligned}
& E\left(e^{2}\right)=E\left[(s+a-y)^{2}\right] \\
& =E\left[s^{2}\right]+2 E[s a]-2 E[s y]+E\left[(a-y)^{2}\right]
\end{aligned}
$$

ECG signal and noise are uncorrelated, so that 


$$
E\left(e^{2}\right)=E\left(s^{2}\right)+E\left[(a-y)^{2}\right]
$$

The smallest possible output power is $E\left[e^{2}\right]=E\left[s^{2}\right]$. When this is achieved, $E\left[(a-y)^{2}\right]=0$. Therefore, $y=a$ and $e=s$. This means minimizing output power causes the output signal to be noise free (Widrow et al. 1975).

Least-Mean-Squares (LMS) adaptive filter was applied in this study. In this thesis work, all the artifacts are reduced offline in Matlab.

\subsection{Least-Mean-Squares Adaptive Filter}

The Least-Mean Squares (LMS) algorithm is an iterative technique for minimizing the mean squared error (MSE) between the primary and the reference inputs. An adaptive filter $\boldsymbol{W}(n)$ can be represented as an $M \times 1$ array of filter coefficients, and $M$ is the order of the filter.

$$
W(n)=\left(\begin{array}{c}
w_{0}(n) \\
w_{1}(n) \\
\cdots \\
w_{M-1}(n)
\end{array}\right), U(n)=\left(\begin{array}{c}
u(n) \\
u(n-1) \\
\ldots \\
u(n-M+1)
\end{array}\right)
$$

$\boldsymbol{W}(n)$ is iteratively updated after each sample according to the following equations.

$$
\begin{aligned}
& y(n)=W^{T}(n) U(n) \\
& e(n)=d(n)-y(n) \\
& W(n+1)=W(n)+\mu U(n) e(n)
\end{aligned}
$$

Where,

$n$ is the current time index

$\boldsymbol{U}(n)$ is the vector of buffered input samples at step $n$ 
$u(n)$ is the optical sensor output at step $n$, served as a auxiliary input $d(n)$ is the noisy ECG signal at step $n$, served as the desired response $y(n)$ is the estimated artifacts at step $n$, served as the filtered output $e(n)$ is the ECG signal with reduced artifacts at step $n$, served as the estimation error

$\mathbf{W}(n)$ is an $M \times 1$ array of estimated filter coefficients at step $n$ (If prior knowledge of the filter coefficients $W(n)$ is available, use it to select an appropriate value for $W(0)$, otherwise set $W(0)=0$.)

$M$ is the order of the filter (filter length; number of taps)

$\mu$ is the step-size parameter 


\section{Chapter 5: Adaptive Artifact Reduction}

\subsection{Methodology}

The proposed sensor system and methodology can be used for either continuous ECG monitoring or ambulatory ECG monitors. Figure 21 (a) illustrates the apparatus for the ECG recording and motion artifact removal system, with all the recorded data analyzed offline in a computer. Figure 21 (b) shows the apparatus for ambulatory ECG recording and motion artifact reduction, with the adaptive filtering algorithm programmed in a microprocessor and the artifacts reduction processed online during recording.

\section{Electrode}

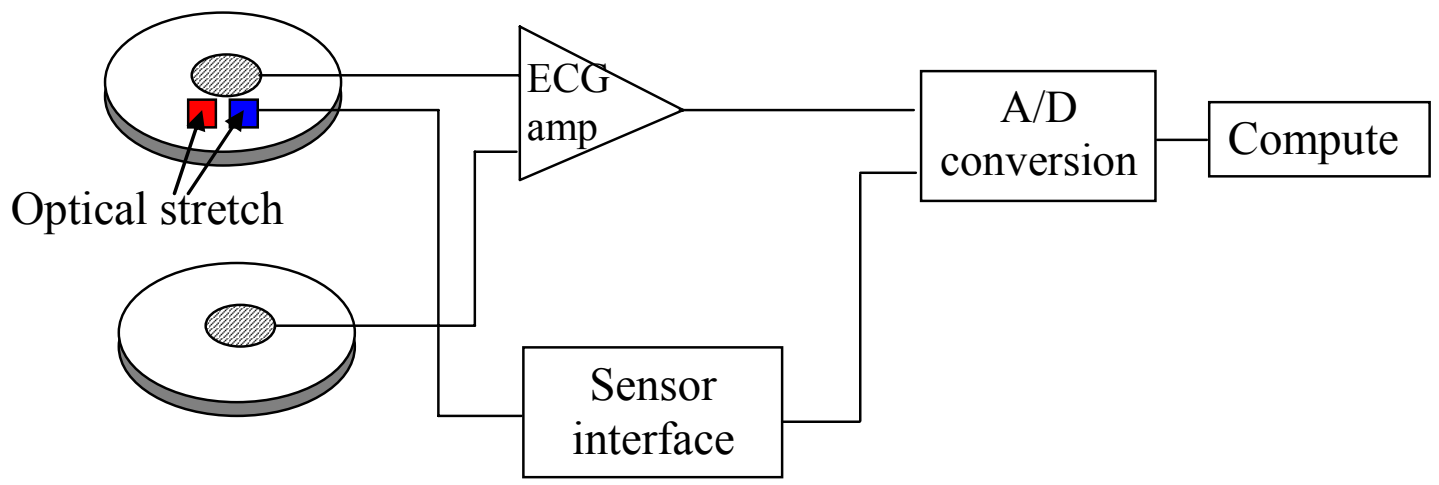

(a) 


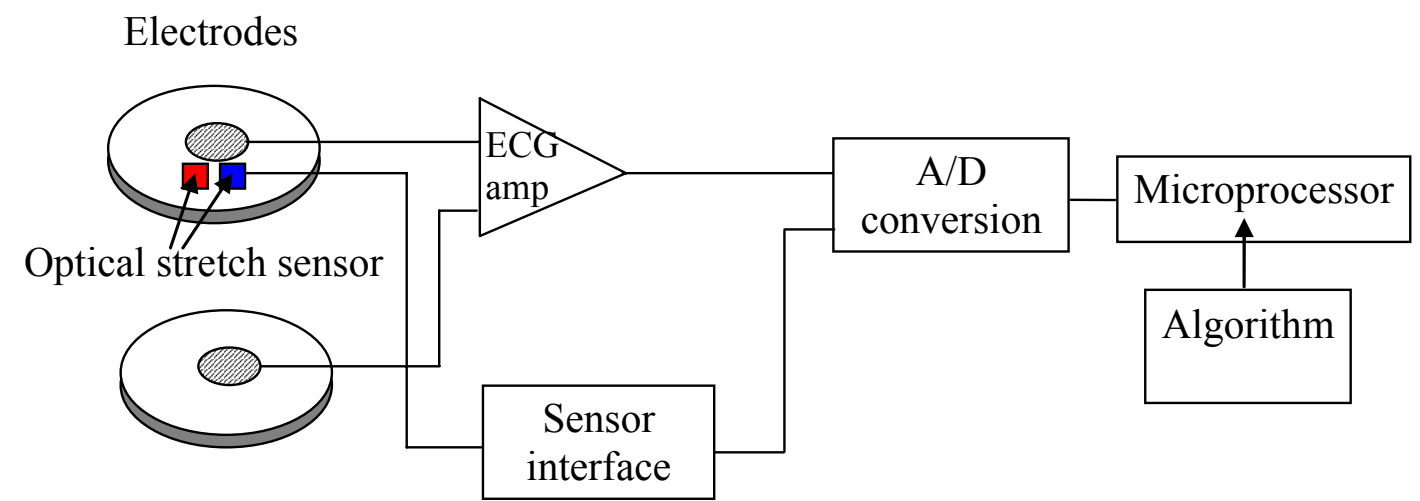

(b)

Figure 21. Apparatus for (a) ECG monitor; (b) ambulatory ECG monitor

In this thesis, the artifacts reduction process was conducted offline with the adaptive filters programmed in Matlab. Least-Mean-Squares (LMS) adaptive filtering algorithm, implemented in Matlab, has been applied in this study.

\subsection{Experiments}

Skin strain was recorded by optical sensor attached in the vicinity of an electrode. Channel I and channel II ECG in standard clinical applications were recorded in this study under an IRB-approved protocol with a standard ECG amplifier (Harvard Apparatus ECG100C). Channel I ECG was recorded as the potential difference between the two forearms, and the optical sensor was attached by doublesided adhesive at the edge of the electrode on the left forearm. Channel II ECG was recorded as the potential difference between the right forearm and the left leg, and the optical sensor was attached at the edge of the electrode on the right forearm. Figure 22 and Figure 23 shows the electrode locations for Lead II and Lead I ECG recording, respectively. 
The ECG sampling rate was $200 \mathrm{~Hz}$ in this study, which allowed entire bandwidth of the skin surface ECG $(0.05$ to $100 \mathrm{~Hz})$ to be captured. The ECG signal was amplified by 5000 and went through a high pass filter with a $0.5 \mathrm{~Hz}$ cutoff frequency. Figure 24 shows the ECG amplifier, electrode and the optical sensor applied in this study. Figure 25 shows the ECG recording software. Skin strain was measured by an optical sensor and recorded simultaneously with ECG. Skin strain was captured at the rate of $64 \mathrm{~Hz}$, and then linearly interpolated to $200 \mathrm{~Hz}$. ECG and skin strain measurements were programmed to start simultaneously by pressing one key in the keyboard. The optical sensor was attached by double-sided adhesive in the vicinity of the electrode on the left forearm. In each case, artifacts were generated by intentionally stretching the skin in the vicinity of the electrode with the optical sensor attached. 


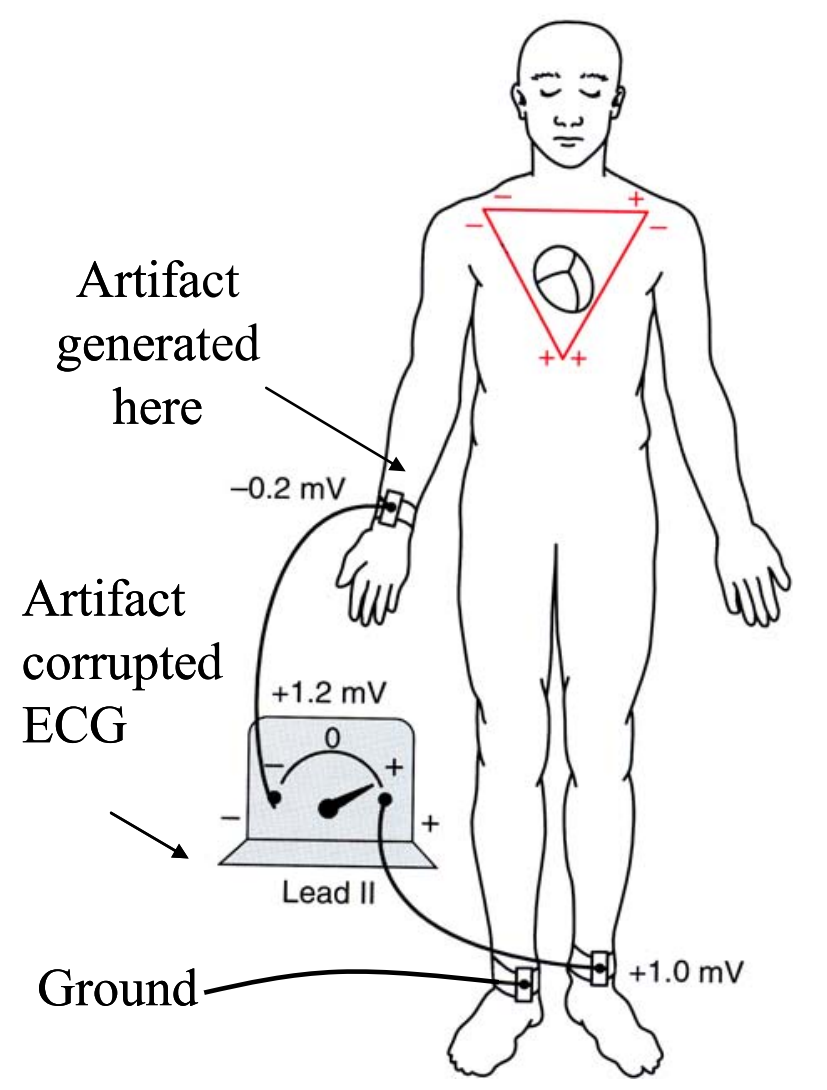

Figure 22. Lead II ECG and skin strain measurement 


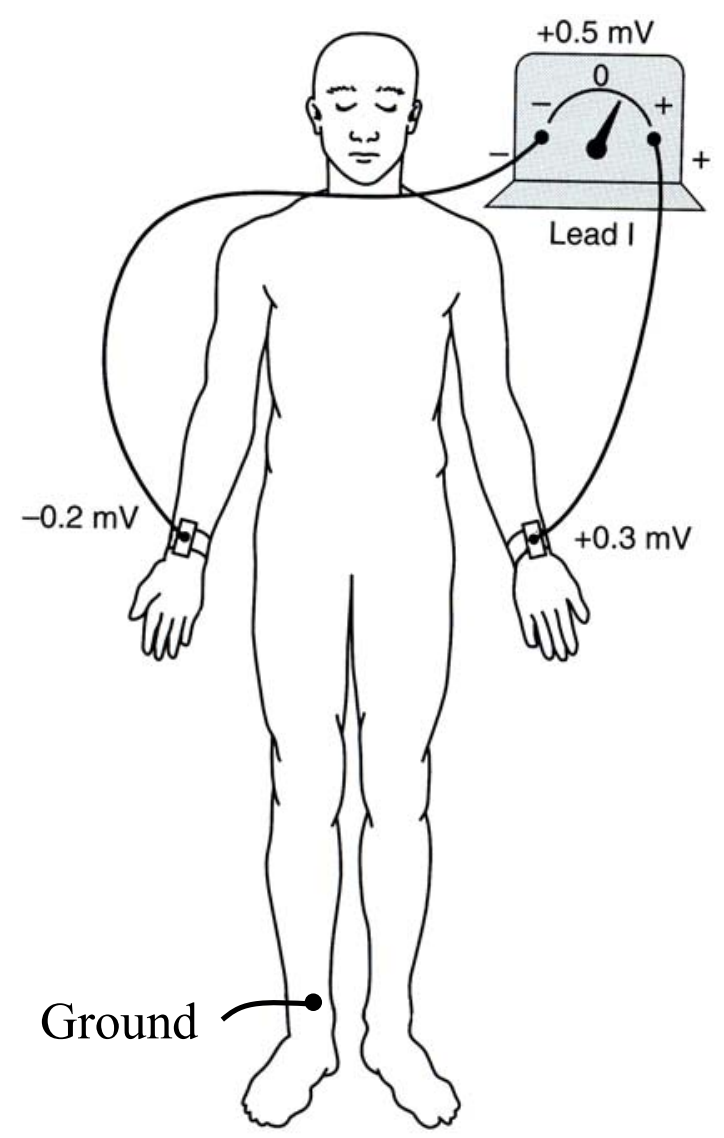

Figure 23. Lead I ECG measurement sites

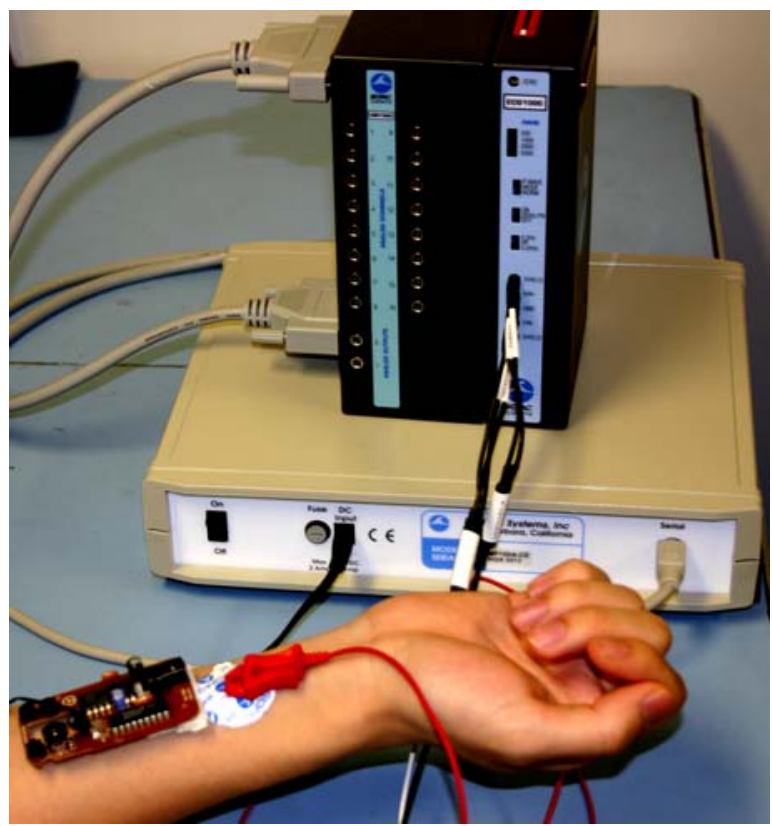


Figure 24. The ECG amplifier, electrodes and the optical sensor

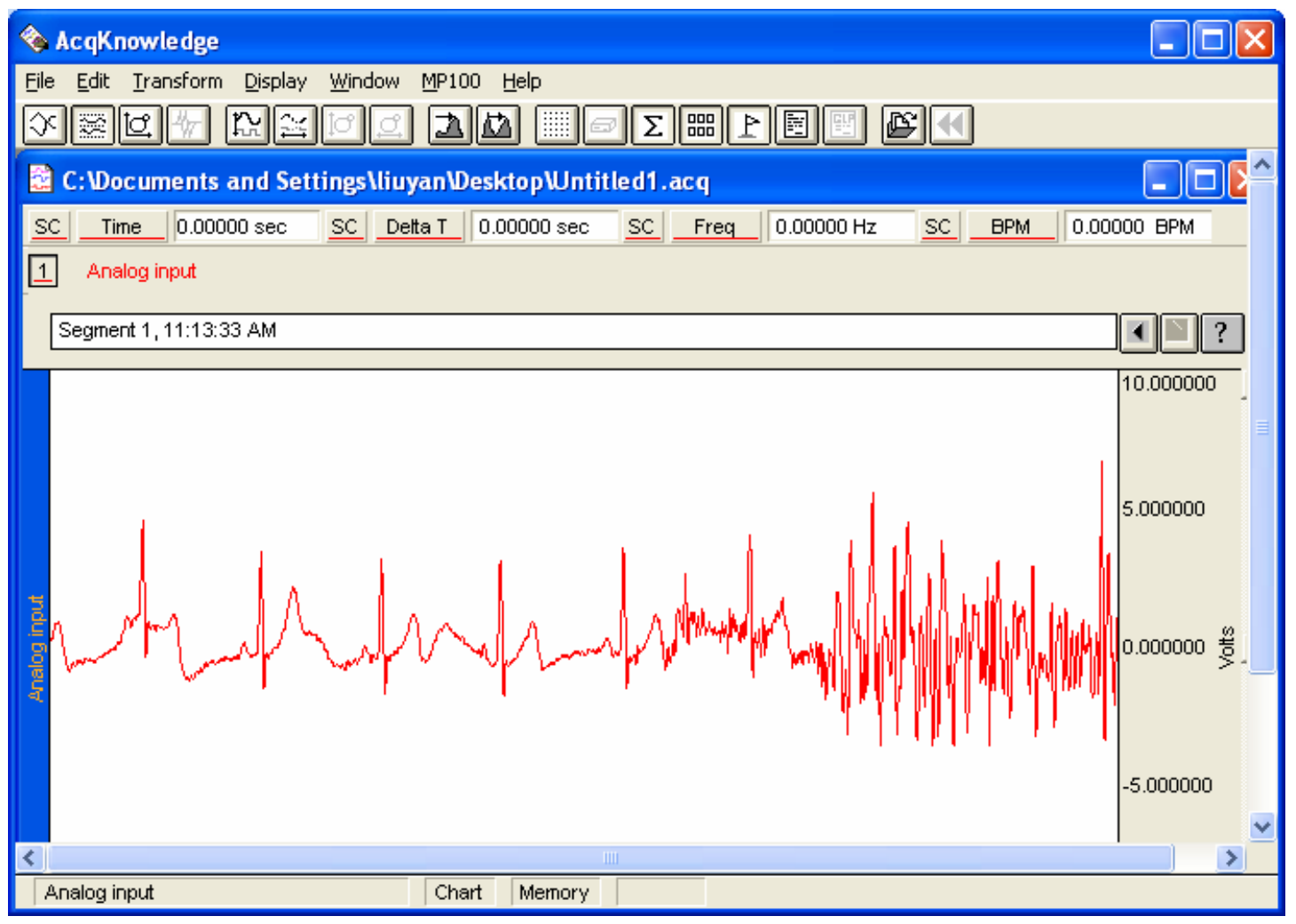

Figure 25. ECG recording software

The optical sensor was connected to a computer by a USB cable. The sensor output was recorded by pressing a key to activate a Delphi program, as shown in Figure 26. Optical sensor output ( $\mathrm{X}$ and $\mathrm{Y}$ offset values) and time are recorded for each sampling point. The optical sensor output $S$ was then calculated by

$$
S=\sqrt{\left(x-x_{0}\right)^{2}+\left(y-y_{0}\right)^{2}}
$$

Where

$x$ and $y$ are the optical sensor output - offset values in $x$ and $y$ directions; 
$x_{0}$ and $y_{0}$ are initial optical sensor output - offset values in $x$ and $y$ directions. The assumption here is that the strain is one dimensional and uniformly distributed underneath the electrode.

The optical sensor output was then linearly interpolated to $200 \mathrm{~Hz}$. The interpolated optical sensor output then went through a high pass filter with the cutoff frequency $1 \mathrm{~Hz}$ to eliminate the offline drift (Butterworth highpass filter, filter order is 8 , passband edge frequency is $1 \mathrm{rads} / \mathrm{sec})$. The filtered optical sensor output signal and ECG signal were saved as data file to serve as the inputs of adaptive filters.

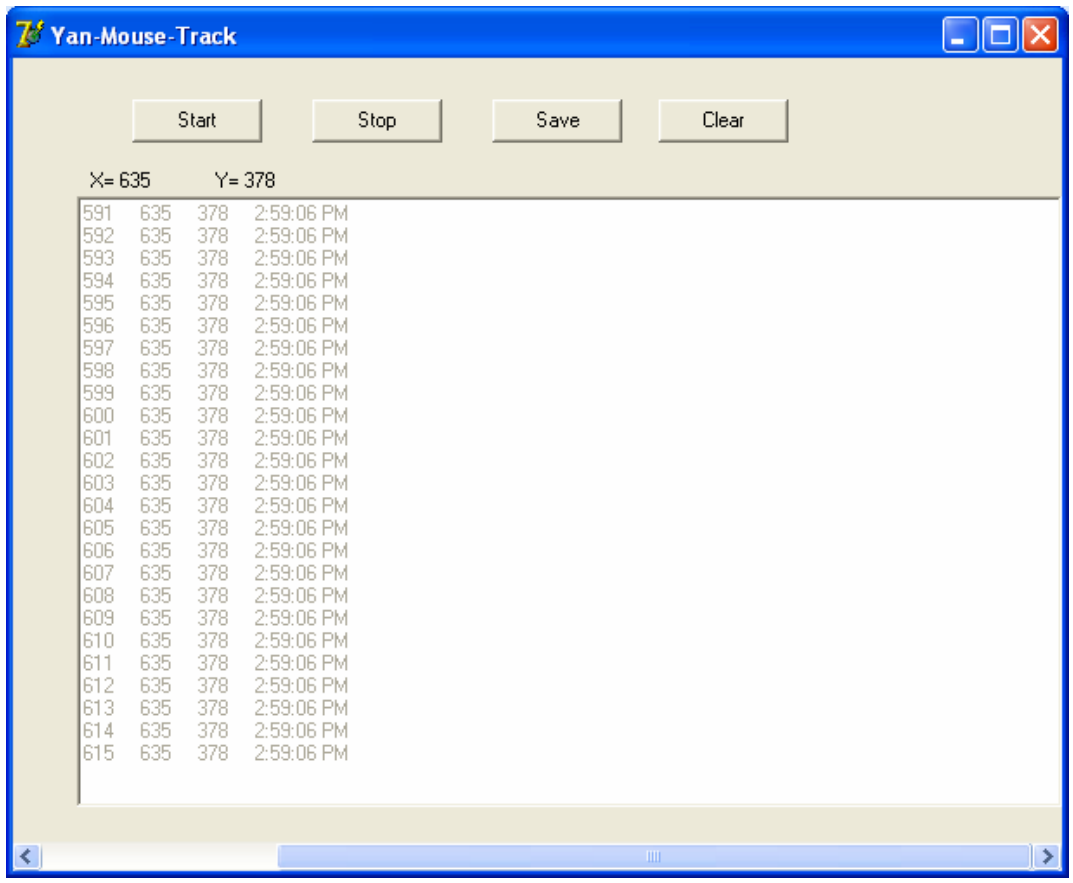

Figure 26. Optical sensor output recording program 


\subsection{ECG Artifacts Reduction}

Figure 27 shows the ECG signal and artifacts. A typical ECG waveform is composed of a P wave, a QRS complex (which is comprised of Q, R and S waves), and a T wave, as shown in the upper plot of Figure 27. The significant features of the waveform are the waves and their individual durations, and time intervals including the P-R, S-T and Q-T intervals (Webster 1998). The bottom plot of Figure 27 is the ECG signal corrupted by skin stretch induced artifacts. The artifacts have similar shape of the T waves.
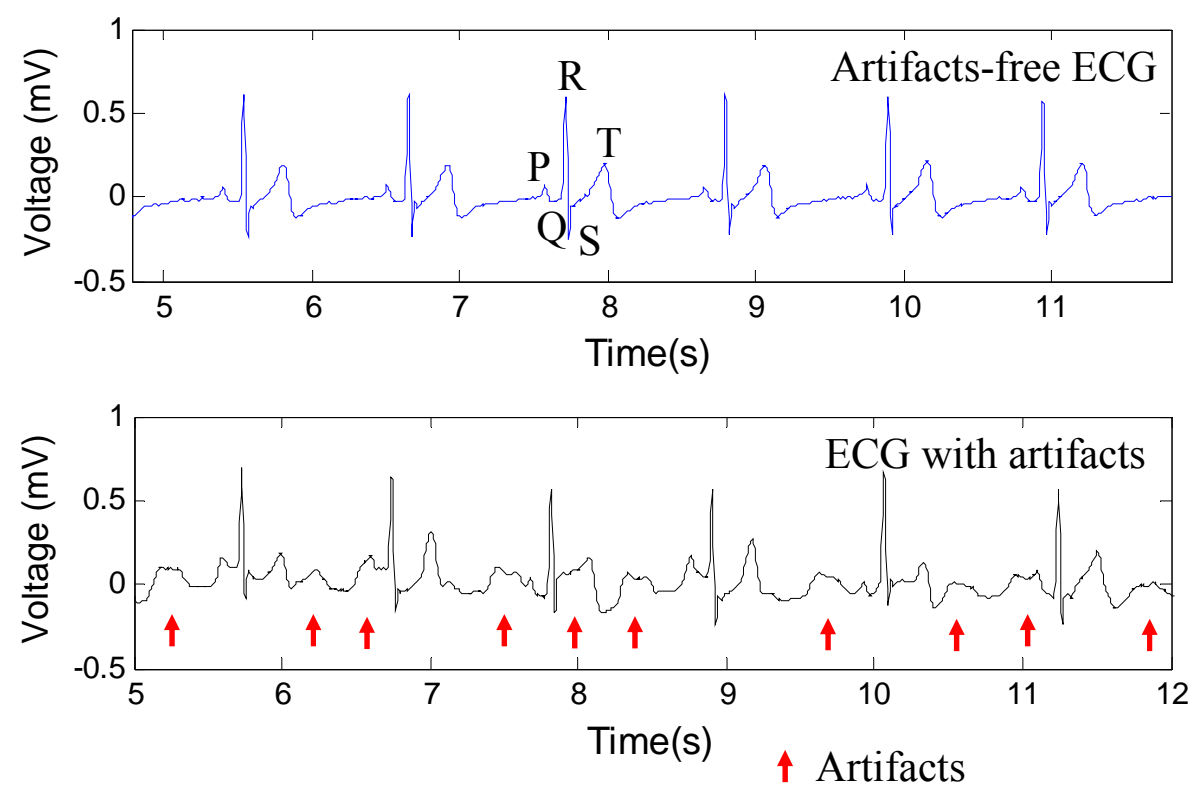

Figure 27. ECG signal and artifacts

Figure 28 shows the artifact reduction in a simple cardiac cycle of the ECG recording. The upper plot of Figure 28 is an artifact-free ECG segment recorded from a human subject. The bottom plot of Figure 28 shows a lead I ECG segment with artifacts from the same subject; and the same signal segment after LMS adaptive 
filtering. It can be shown that the adaptive filter reduced the skin stretch induced artifacts, while keeping the useful waveforms, compared with the artifact-free ECG segment.
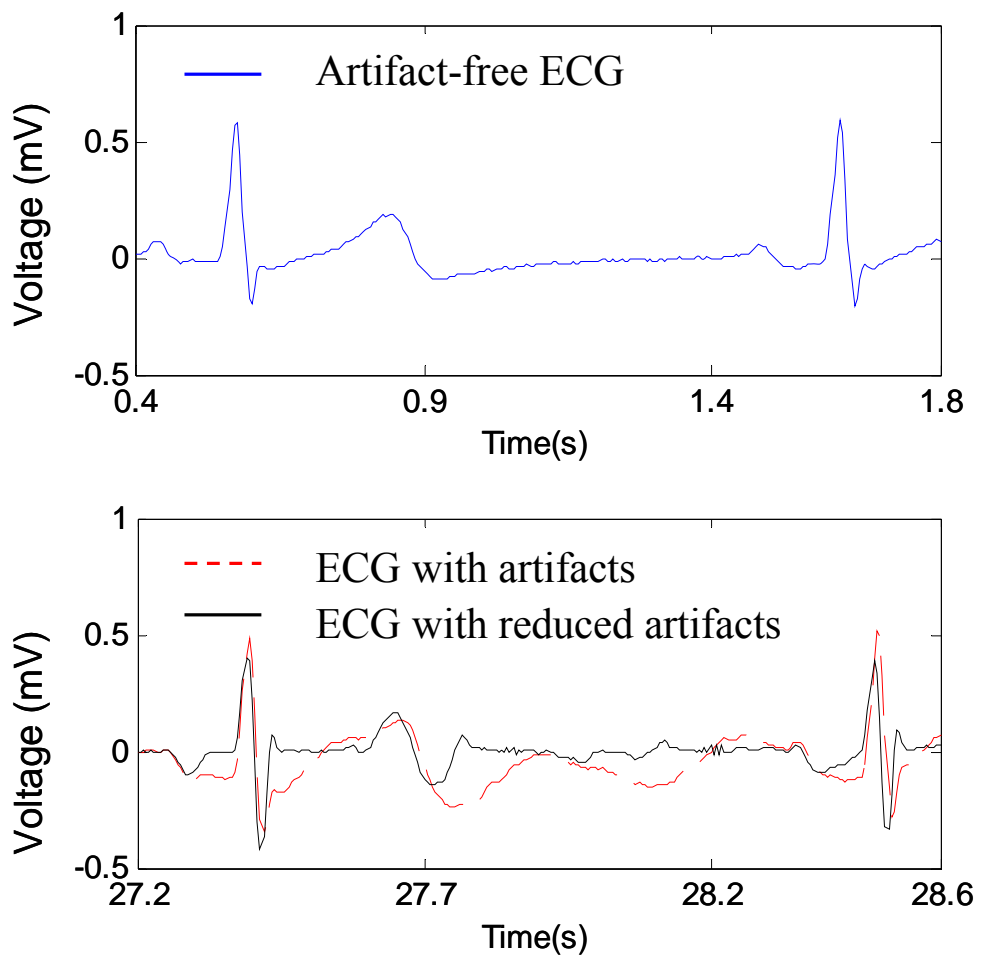

Figure 28. One cardiac cycle of lead I ECG artifact reduction

Figure 29 shows the artifact reduction results of a 30 -second lead I ECG recording. The four traces in Figure 29 are optical sensor output which indicates the amount of skin strain, estimated artifacts by the adaptive filter, ECG signal before artifact reduction, and ECG signal after artifact reduction. The first three cardiac cycles were artifact-free. Comparing artifact-free ECG in the first three cardiac cycles with the following noisy ECG, it is shown in Figure 29 that a large amount of artifacts were picked by the adaptive filters results in artifact reduced ECG signals. 

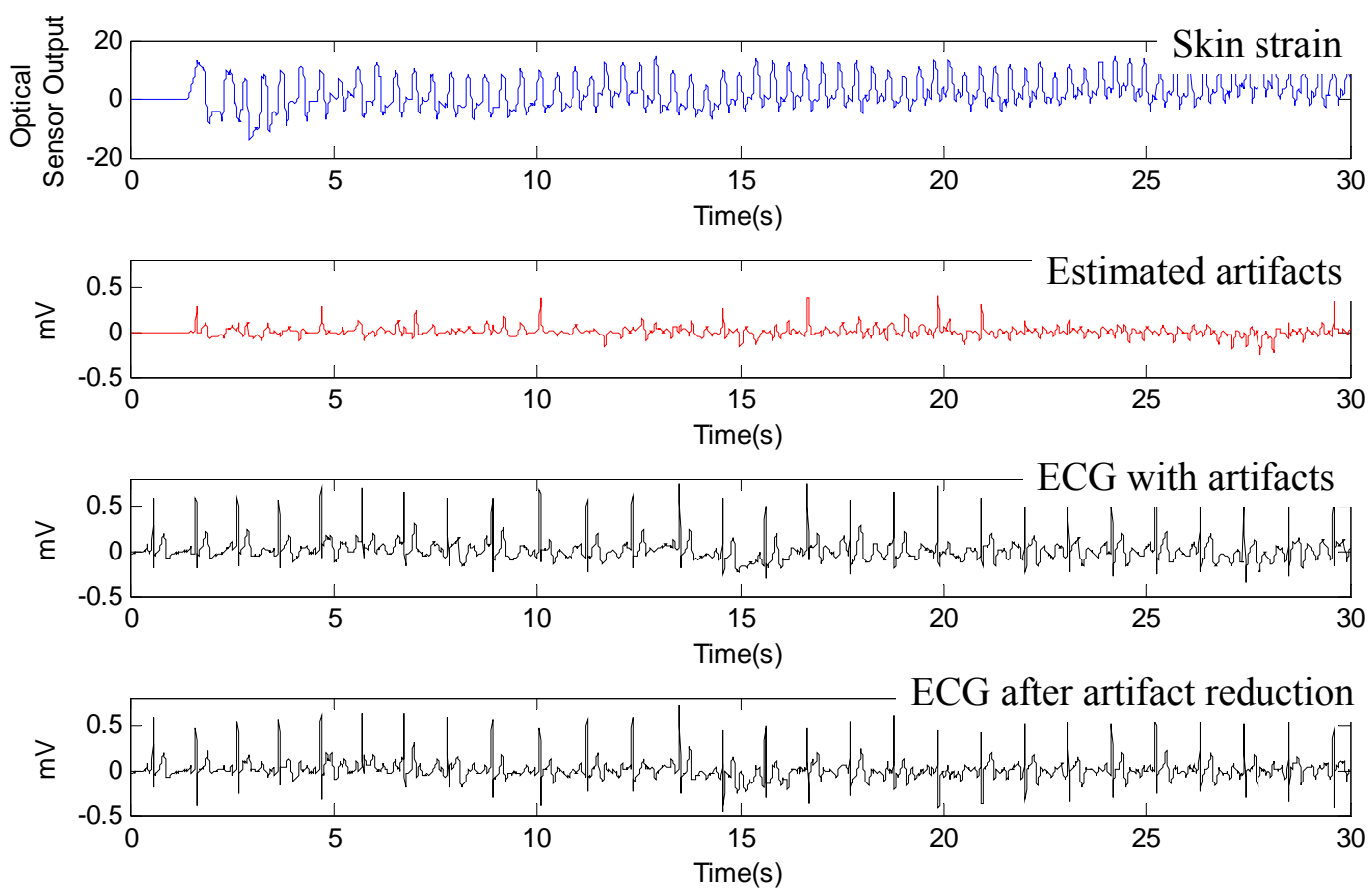

Figure 29. Artifact reduction of a 30-second lead I ECG data

Lead II ECG recording and skin strain signal were shown in Figure 30. The first two cardiac cycles were artifact-free. The locations of artifacts were identified by red arrows. Different from artifacts in lead I ECG, which are mainly adding a positive potential signal to the ECG signal, artifacts in lead II ECG generally adding a negative potential signal to the ECG data. This is because the potential on the right forearm is smaller than that of the left leg, and skin stretch on the right forearm causes its potential to increase, the acquired ECG signal showed a significant decrease when the skin around the electrode was stretched. Therefore, the 10 large negative peaks identified by red arrows in the second trace of Figure 30 were artifacts due to skin stretch. 

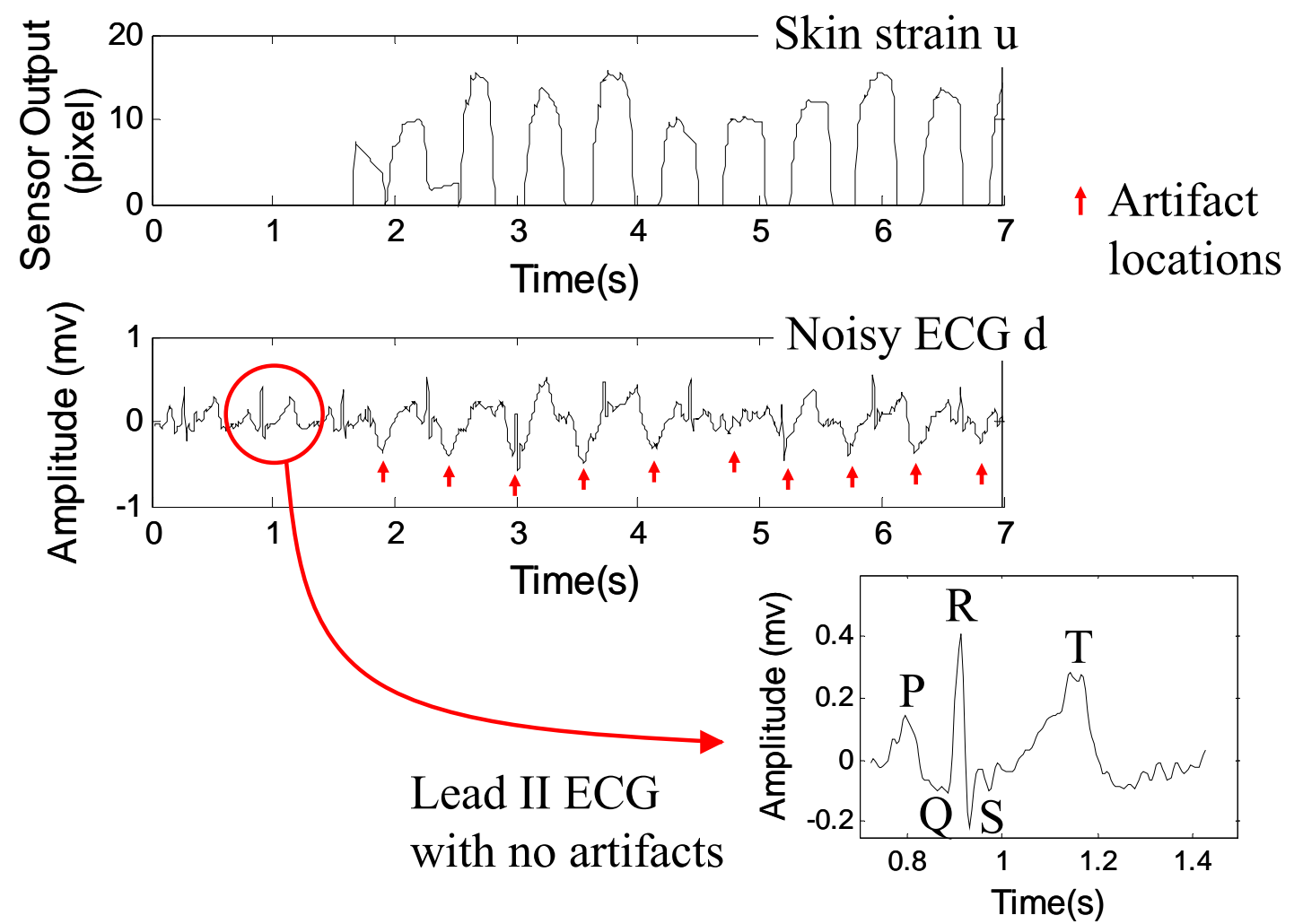

Figure 30. Lead II ECG and skin strain induced artifacts

Figure 31 shows the artifacts reduction results of the lead II ECG signal. In

Figure 31, the third trace (ECG signal corrupted by artifacts) contained 10 negative peaks which were skin strain induced artifacts during 7 seconds. In the fourth trace (estimated ECG after noise removal), all the 10 large negative peaks were eliminated except for the first one, while the positive ECG features in the second trace were kept the same. The results demonstrated that the proposed sensor systems and method can significantly reduce ECG artifacts generated by skin strain. 

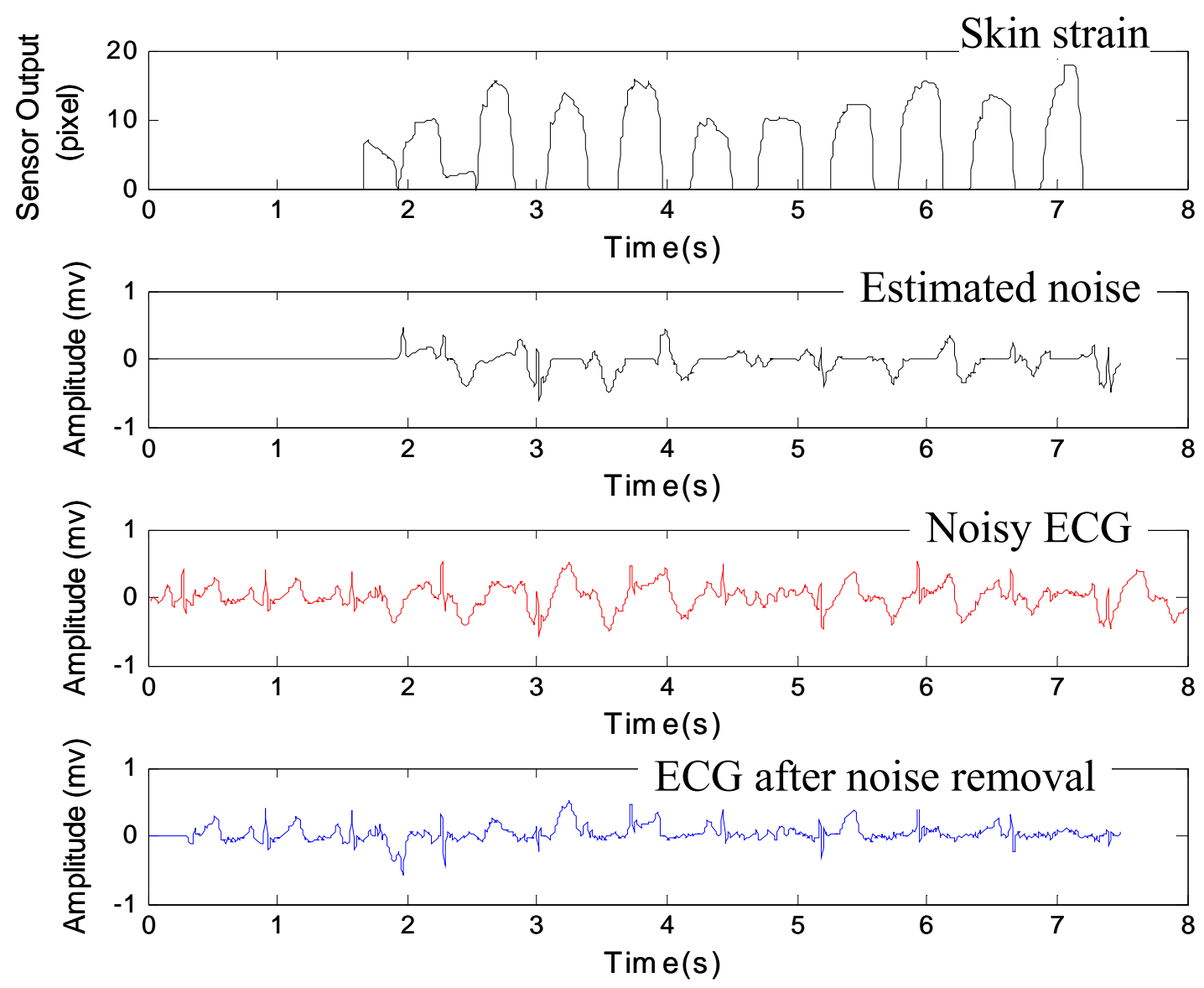

Figure 31. Lead II ECG artifacts reduction

It was observed during measurement that different human subjects have different ECG amplitudes and skin stretch induced potentials. This observation is consistent with Odman's study $(1981,1982)$, which indicated that measured skin stretch potentials showed large individual variations. Odman's data showed that for stretched skin potentials, standard deviations are in the range of $32 \%-94 \%$ of the corresponding mean values.

For some individuals who have stronger ECG signal, skin stretch induced artifacts can be comparatively smaller. For other individuals who have weaker ECG signals, skin stretch induced artifacts can have larger amplitudes than that of the ECG 
signal. One example is illustrated in Figure 32. Figure 32 (a) and Figure 32 (b) are recorded from human subject a and b, respectively. In both (a) and (b), the upper plot is the artifact-free ECG, and the bottom plot is the ECG corrupted by skin stretch induced artifacts.

In Figure 32 (a), the peak value of subject a's ECG QRS complex is approximately $0.6 \mathrm{mV}$, while skin stretch induced artifacts have the amplitude of 0.1$0.2 \mathrm{mV}$, which is comparable to the amplitudes of the T waves. In Figure 32 (b), the peak value of subject b's ECG QRS complex is less than $0.1 \mathrm{mV}$, while skin stretch induced artifacts have the amplitude of approximately $0.2 \mathrm{mV}$, which is larger than the QRS complex amplitude. Even though the amplitudes of ECG and skin strain induced voltage are different from person to person, skin strain induced ECG artifacts can be reduced by this method, since the filters adaptively build the equation between skin strain signal and ECG motion artifacts. In other words, the filter adapts itself to describe the relationship between skin strain signal and ECG artifacts for a particular individual. 

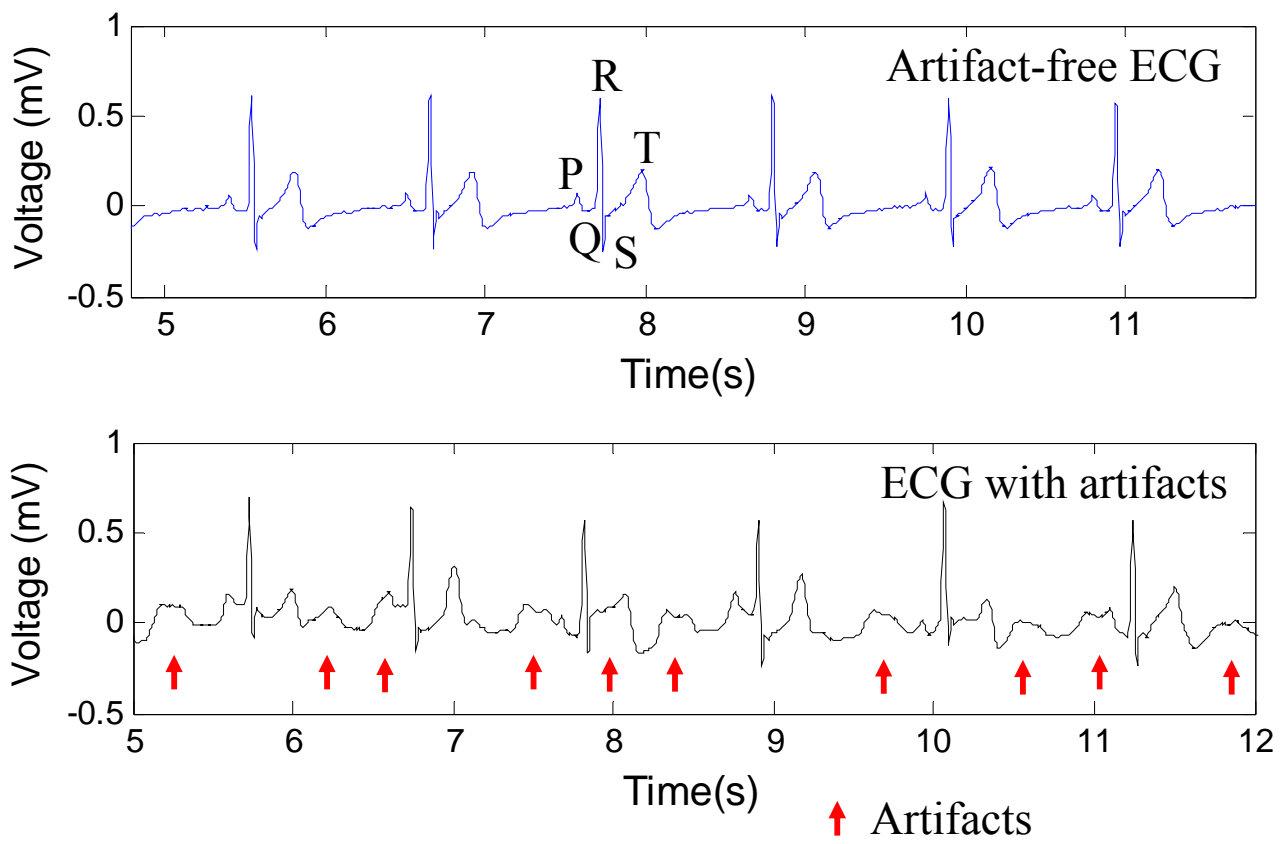

(a)
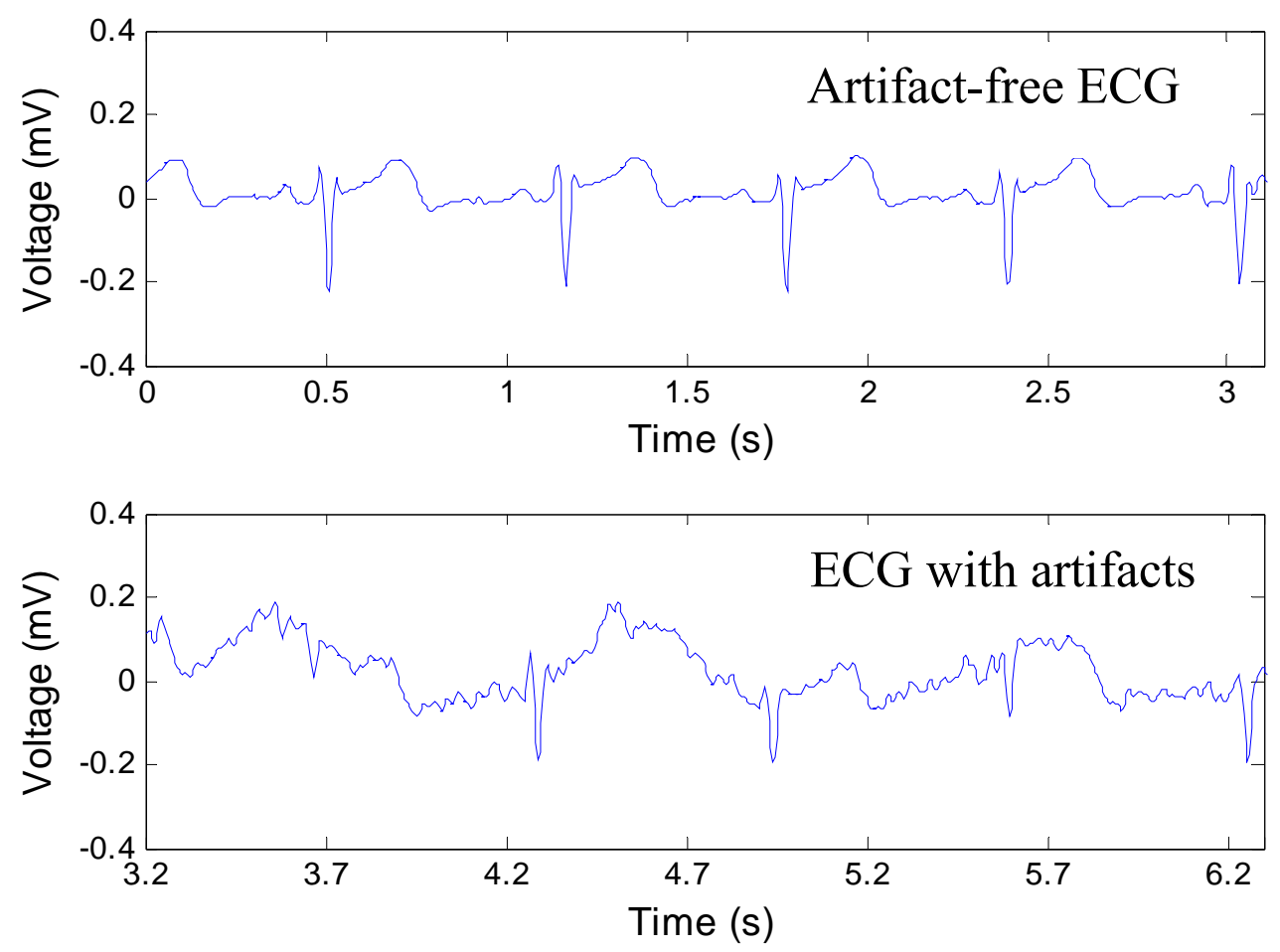

(b)

Figure 32. (a) Artifact-free ECG and ECG with artifacts for subject a; (b) Artifactfree ECG and ECG with artifacts for subject $b$ 
During the experiments, it was observed that even for the same human subject, stretching the skin may not necessarily induce ECG artifacts. In other words, a subject's skin stretch induced artifact may be obvious for a certain period of time, and disappear for another period of time. The reason hasn't been clearly reported in literature. Odman (1981) observed that time sequences for the skin stretch potentials vary individually. Studies of Tam and Webster (1977), and Burbank and Webster (1978) mainly focused on the influence of abrasion and of electrolyte concentration on skin induced motion artifact in biopotential recording. Thakor and Webster (1978) hypothesized that the skin potential arises from a constant current source called 'injury current' flowing through the extracellular resistance. de Talhouet and Webster (1996) validated Thakor's model through experiments and hypothesized that another possible explanation would be a direct influence of the stretch on the active metabolic process. Up to now there is no widely accepted explanation on why skin strain induces skin voltage change. Since adaptive filters describes a dynamic relationship between skin strain signal and ECG artifacts, this phenomena is not a concern in this study. For the time period when stretching skin causes no or little artifacts, the filter weights will be adjusted to zero or close to zero, to indicate that artifacts are negligible in this time frame.

Another alternative of our approach is that a two dimensional analysis can be conducted. This is necessary because skin is an anisotropic material. Skin tends to be much stiffer along Langer's lines ${ }^{37}$ rather than across them. Concerning electrical properties, according to Odman's (1982) observations, it can be concluded that skin presents isotropic electrical properties, meaning that the skin stretch induced artifacts

\footnotetext{
${ }^{37}$ In surgery, the preference directions of skin related to stress are called Langer's lines.
} 
only depend on the amount of skin strain, but don't depend on skin stretch directions. In this study the optical sensor output $\mathrm{S}$ indicates the skin strain magnitude as a function of time, without the information of skin strain directions.

\subsection{Artifact Reduction Quantification}

At this time, there is no standard method to quantify the amount of artifacts eliminated. Thus signal processing methods were used in this study to quantify the amount of artifacts eliminated. A 30-second artifact-free ECG signal was recorded with the human subject at rest. A 30-second noisy ECG was recorded on the same human subject right after the noisy-free ECG, so that the heart rate was approximately the same and therefore the noisy-free ECG data set can be used as an "ideal" reference.

The $\mathrm{L}_{2}$ norm of the noisy ECG data and the data after artifact removal was

calculated, was used as the metric for noise reduction. The more artifacts remain in ECG signal, the larger the L2-norm of the noisy ECG signal. The L2-norm of a vector

$$
\begin{aligned}
& x=\left[\begin{array}{c}
x_{1} \\
x_{2} \\
\cdots \\
x_{k}
\end{array}\right] \\
& \text { is }|x|=\sqrt{\sum_{k=1}^{n}\left|x_{k}\right|^{2}}
\end{aligned}
$$

A percentage improvement statistic was calculated as Artifact Reduction Percentage $=$ 


$$
\frac{L_{2 \_ \text {before }}-L_{2 \_ \text {after }}}{L_{2 \_ \text {before }}-L_{2 \_ \text {ideal }}} \times 100 \%
$$

Where $L_{2 \_ \text {before }}$ is the L2-norm of the noisy ECG signal; $L_{2 \_ \text {after }}$ is the L2-norm of the ECG signal after artifact reduction; $L_{2 \_ \text {ideal }}$ is the L2-norm of the artifact-free ECG signal.

A 30-second ECG signal contained approximately 50 cardiac cycles. It was found that the artifact reduction percentage calculated using 10 -second ECG signals was approximately the same as that calculated by using 30 -second ECG signals. Thus a 30-second ECG signal is considered capable of providing accurate results for artifact reduction percentage analysis.

Table 2 shows the artifact reduction performance of lead I ECG. Three recordings of lead I ECG were recorded for each of three individuals. Every recording lasts 30 seconds, i.e., 6000 data points. As mentioned in section 5.2, artifacts were generated by intentionally stretching the skin on the left forearm. The filter order was set as 10 , and the initial filter coefficients were set as zero. The results showed that on average of the nine 30-second recordings, LMS can reduce $83 \%$ of motion artifacts.

Table 2. Artifact reduction results of lead I ECG

\begin{tabular}{|c|c|c|}
\hline Subject & No. & Artifact Reduction (\%) \\
\hline \multirow{2}{*}{1} & 1 & 99.56 \\
\cline { 2 - 3 } & 2 & 99.41 \\
\cline { 2 - 3 } & 3 & 97.35 \\
\hline
\end{tabular}




\begin{tabular}{|c|c|c|}
\hline \multirow{2}{*}{2} & 1 & 50.09 \\
\cline { 2 - 3 } & 2 & 34.66 \\
\cline { 2 - 3 } & 3 & 65.96 \\
\hline 3 & 1 & 98.66 \\
\cline { 2 - 3 } & 2 & 99.48 \\
\cline { 2 - 3 } & 3 & 99.63 \\
\hline
\end{tabular}

The difference in artifact reduction performance for different individuals can be attributed to shape feature difference of the skin area where the optical sensor is attached. Non-flat attaching surface or larger curvature of the sensor mounting spot can cause the optical sensor to be out of focus during motions, which can lead to inaccurate sensor output for some time frame. To ensure a proper sensor attachment to the skin surface during motions is found to be the key challenge in this study, which otherwise can cause signal instability of the optical sensor output. Non-flat skin / body surface, vertical deformation of the skin, or vertical movement of the sensor may cause the image to lose focus. Plane imaging should be maintained to satisfy the plane-strain state. A further device miniaturization is suggested to ensure better attachment on different geographic skin surface.

\subsection{Tests of Ambulatory Conditions}

To further assess the artifact reduction performance of the apparatus and method for ambulatory ECG monitors, the sensor system was tested under various types of motions that mimic everyday activities, including running, walking, raising 
the arm vertically, waving the arm horizontally and slightly stretching the upper chest. A modified lead I ECG was recorded during ambulatory testing. Electrode sites and the optical sensor site were shown in Figure 33. Two electrodes were attached to the subject's upper chest, with one on the left and one on the right. The optical sensor was attached in the vicinity of the electrode on the upper left chest.

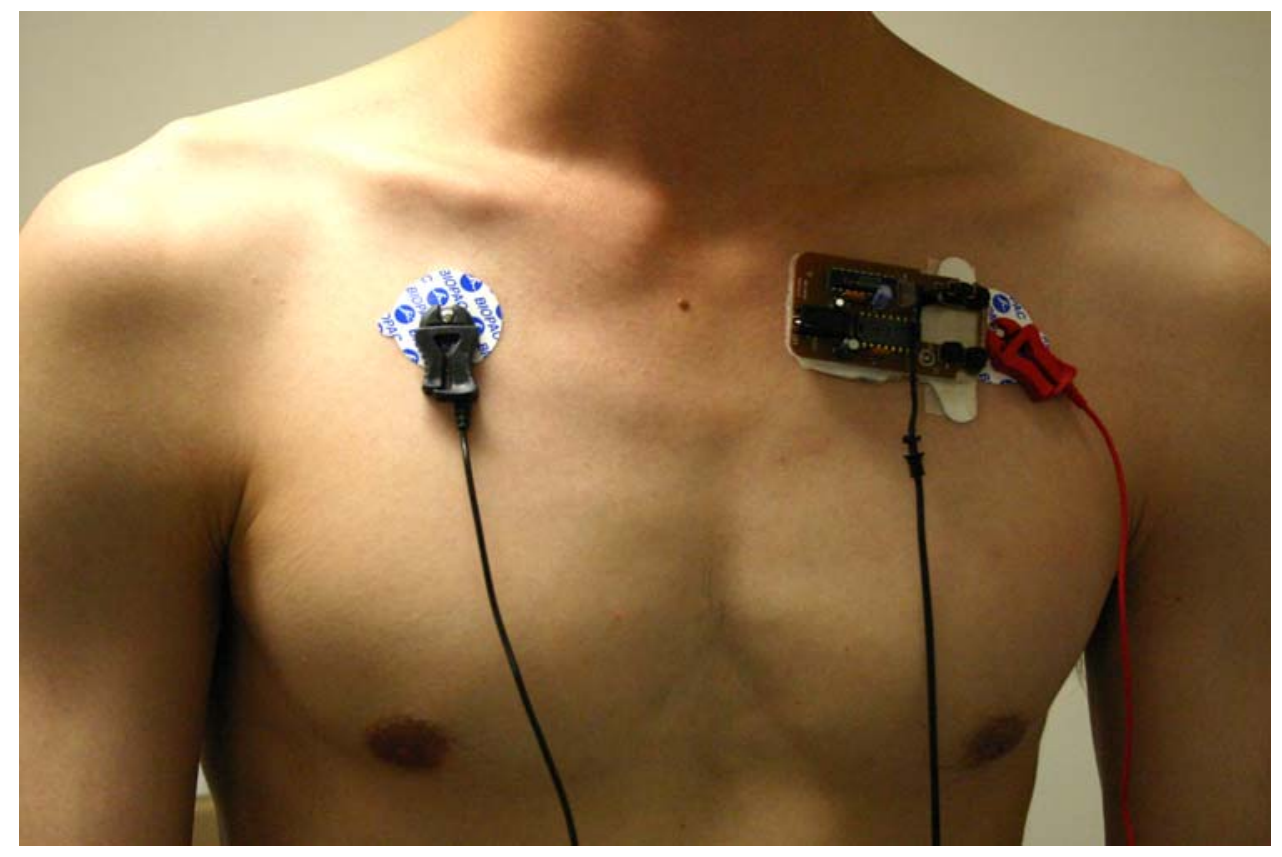

Figure 33. Ambulatory testing setup

In total there were 15 recordings made from one human subject. Every recording lasts 30 seconds. Artifacts reduction performance of ambulatory ECG was quantified by L2 Norm, with the results shown in Table 3. The filter order was set as 10 , and the initial filter coefficients were set as zero. On average, the LMS filter reduced $85 \%$ of the artifacts. The worst artifact performance can be attributed to nonflat skin / body surface, vertical deformation of the skin, or vertical movement of the 
sensor that may cause the image to lose focus. The worse case was found when the subject horizontally waved the left arm, which caused part of the optical sensor to lift from the skin surface. However, even for this worst case, the method achieved an artifact reduction of $43 \%$. To ensure a proper sensor attachment to the skin surface during motions, device miniaturization is suggested.

Table 3. Artifact reduction results of ambulatory testing

\begin{tabular}{|c|c|c|}
\hline No. & Motion Type & Artifact Reduction (\%) \\
\hline 1 & \multirow{2}{*}{$\begin{array}{l}\text { Slightly stretching } \\
\text { the upper chest }\end{array}$} & 77.37 \\
\hline 2 & & 83.62 \\
\hline 3 & \multirow{3}{*}{$\begin{array}{c}\text { Horizontally } \\
\text { waving the left arm }\end{array}$} & 97.80 \\
\hline 4 & & 43.23 \\
\hline 5 & & 56.31 \\
\hline 6 & \multirow{3}{*}{ Vertically raising } & 99.65 \\
\hline 7 & & 98.78 \\
\hline 8 & & 98.89 \\
\hline 9 & \multirow{4}{*}{ Walking } & 99.44 \\
\hline 10 & & 99.88 \\
\hline 11 & & 99.27 \\
\hline 12 & & 99.91 \\
\hline 13 & \multirow{3}{*}{ Running } & 55.26 \\
\hline 14 & & 78.14 \\
\hline 15 & & 80.75 \\
\hline
\end{tabular}


Average (standard deviation)

$84.55(19.26)$

It should be noted that even though this method can reduce $55-81 \%$ artifacts under running conditions, since the total amount of noise is much larger than other conditions, the running ECG after artifact reduction is still quite noisy (see Appendices). Under running conditions it is more challenging to ensure the optical sensor signal stability and accurately capture the skin strain data. So even if this method can reduce more than half of the total artifacts under running conditions, the large amount of remaining noise still corrupts the ECG signal, and makes this method less effective under running conditions compared with other conditions.

Figure 34 shows the artifact reduction results of a 30-second ambulatory ECG test. The data was recorded as the subject stretched the upper chest (recording 1 in Table 3). The first few cardiac cycles were artifact-free (in the first 3.6 seconds), when the subject was at rest. Trace 1 to 4 are the optical sensor output, estimated artifacts, ECG before artifact reduction, and ECG after artifact reduction, respectively. The optical sensor output indicates the subject's motion as he repeatedly stretched and relaxed his chest, with each peak corresponding to a stretch. In trace 3 of Figure 34, motion artifacts were identified with their locations marked by arrows. The artifacts were identified since their peak amplitudes were generally three times larger a typical $\mathrm{T}$ wave peak amplitude, which is $0.058 \pm 0.005 \mathrm{mV}$ for this subject. The majority of the artifacts were eliminated after adaptive filtering (trace 4 in Figure 34), although a few remained in ECG (trace 4 in Figure 34). The L2-norm calculation showed that $77 \%$ of artifacts have been reduced in this recording. 
Artifact reduction performance for the data in Figure 34 was analyzed cycle by cycle, as shown in Table 4 . There were 50 cardiac cycles in this 30 -second ECG data. In the analysis, the maximum positive value between the two adjacent QRS complex $^{38}$ in each cardiac cycle was recorded. The artifacts have similar morphologies of $\mathrm{T}$ waves. Since $\mathrm{T}$ wave peak value is the maximum positive value between two adjacent QRS complex in an artifact-free cardiac cycle, measuring the maximum positive value between every two QRS complex and comparing it to artifact-free $\mathrm{T}$ wave peak value can give us insights of whether the wave is an artifact, as the artifacts have larger amplitudes than T waves. Similarly, we can find how well the artifact is reduced. A standard artifact-free $\mathrm{T}$ wave peak value was calculated to be $0.0575 \mathrm{mV}$, by averaging the $\mathrm{T}$ wave peak values in the first 4 artifact-free cardiac cycles. Let $T_{0}$ be the standard artifact-free $T$ wave peak value, and let $T_{i}$ be the positive peak value between two adjacent QRS complex in any given cardiac cycle, the noise is defined as a percentage of:

$$
\text { Noise }=\left(\mathrm{T}_{\mathrm{i}}-\mathrm{T}_{0}\right) / \mathrm{T}_{0} * 100,(\mathrm{i}=1 \text { to } 50)
$$

The artifact reduction for each cardiac cycle is defined as the ratio of noise before artifact reduction and noise after artifact reduction $(* 100)$. The artifact reduction in the ECG of each cardiac cycle was calculated over 39 cardiac cycles in a 30-second record, as shown in Table 4 (For the other 11 cardiac cycles, no artifacts were generated). It can be concluded that artifacts were greatly reduced (reduced more than $60 \%$ ) for some cardiac cycles. But for other cardiac cycles the artifact

\footnotetext{
${ }^{38}$ In every cardiac cycle there is a P wave, a QRS complex, and a T wave. QRS complex, consisted of the $\mathrm{Q}, \mathrm{R}$, and $\mathrm{S}$ waves, is caused by ventricular depolarization. The duration is normally 0.06 to 0.1 seconds, and the morphology is more like a spike rather than a rounded wave.
} 
reductions were relatively small (reduced less than $20 \%$ of the artifacts). This indicated that adaptive filtering algorithm does reduce but can't totally eliminate the motion artifacts. For a few cardiac cycles, the artifact reduction percentage was calculated to be larger than $100 \%$, which means that the filter not only reduced artifacts, but also eliminated certain amount of true ECG signals.
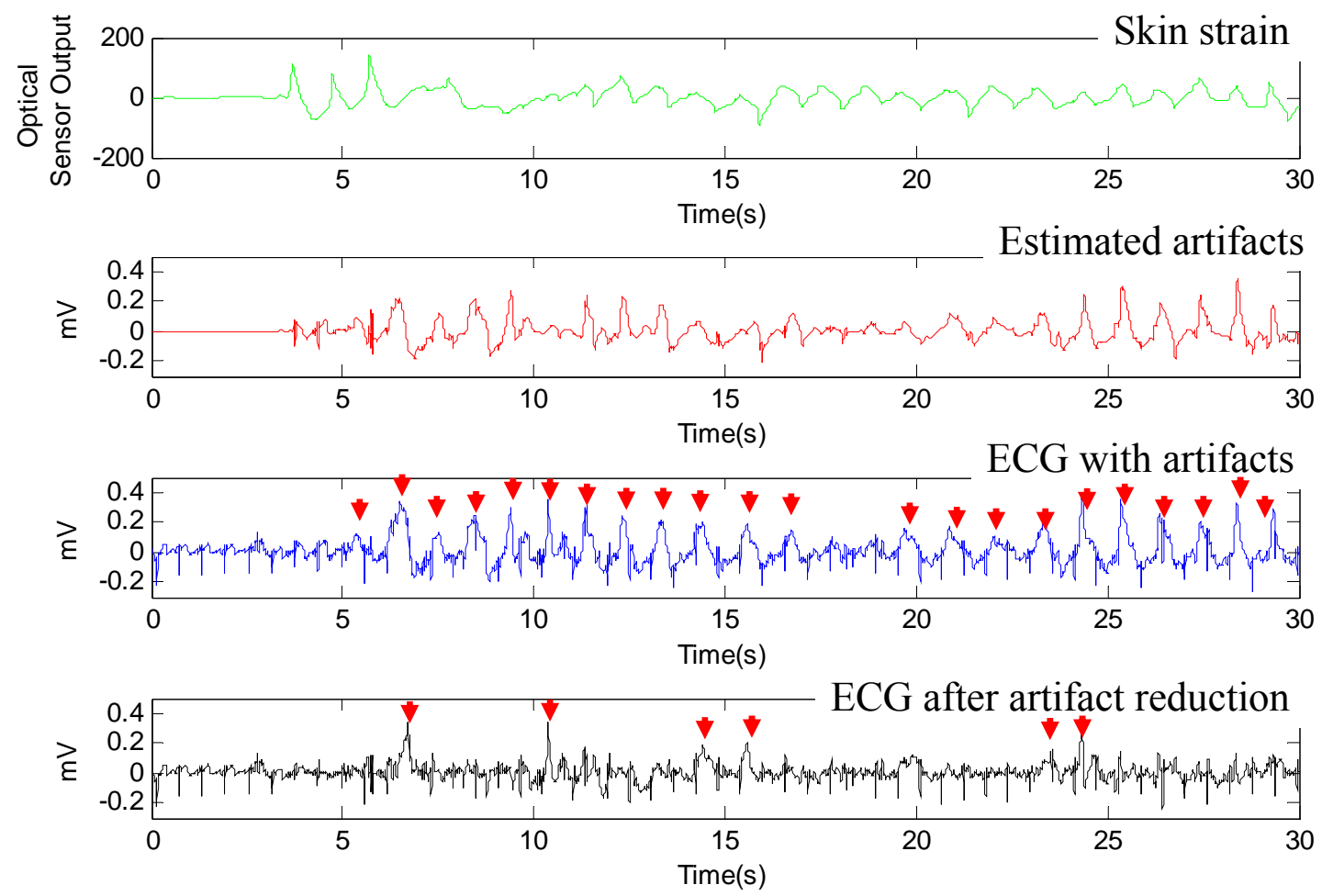

Figure 34. Ambulatory ECG motion artifact reduction (recording 1: subject is slightly stretching the upper chest)

Table 4. Artifact reduction results cycle by cycle (Recording 1 of ambulatory testing: subject is slightly stretching the upper chest)

\begin{tabular}{|l|l|}
\hline Artifact Reduction & Cycles \\
\hline
\end{tabular}




\begin{tabular}{|c|c|}
\hline Percentage & \\
\hline$<0$ & $10(25.6 \%)$ \\
\hline $0-20$ & $3(7.7 \%)$ \\
\hline $20-40$ & $3(7.7 \%)$ \\
\hline $40-60$ & $3(7.7 \%)$ \\
\hline $60-80$ & $5(12.8 \%)$ \\
\hline $80-100$ & $5(12.8 \%)$ \\
\hline $100-120$ & $6(15.4 \%)$ \\
\hline$>120$ & $4(10.3 \%)$ \\
\hline
\end{tabular}

Figure 35 shows the artifact reduction results for another 30 -second ambulatory ECG when the subject was raising the left arm (recording 6 in Table 3). The first few cardiac cycles were artifact-free, when the subject was at rest. Almost all the artifacts were successfully picked up by the adaptive filter and subtracted from the noisy ECG. The L2-norm calculation shows that the artifacts have been reduced by $99 \%$. Figure 36 zoomed in one part of ECG signal in Figure 35. 

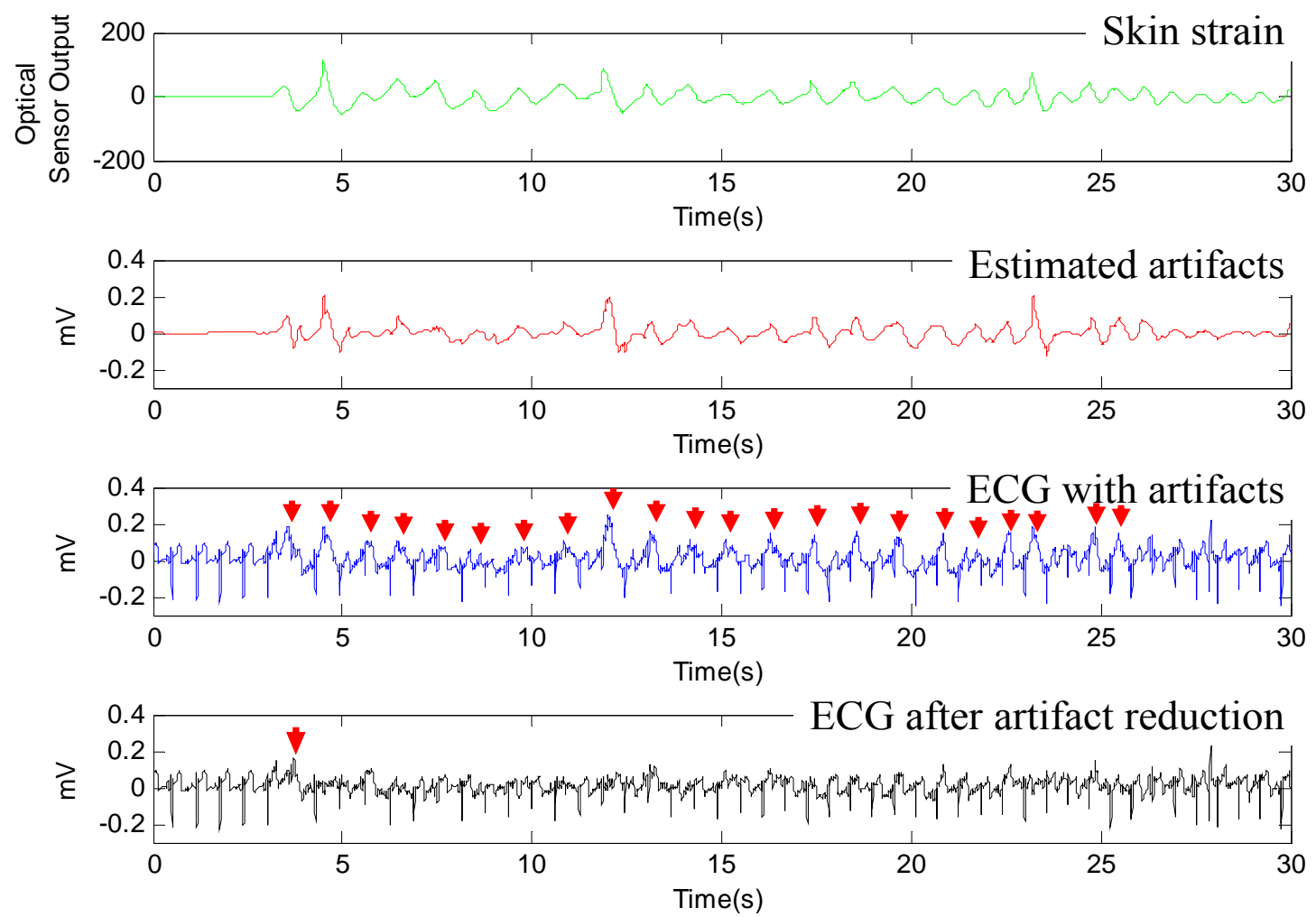

Figure 35. Ambulatory ECG motion artifact reduction (recording 6: subject is raising the left arm)

ECG with artifacts
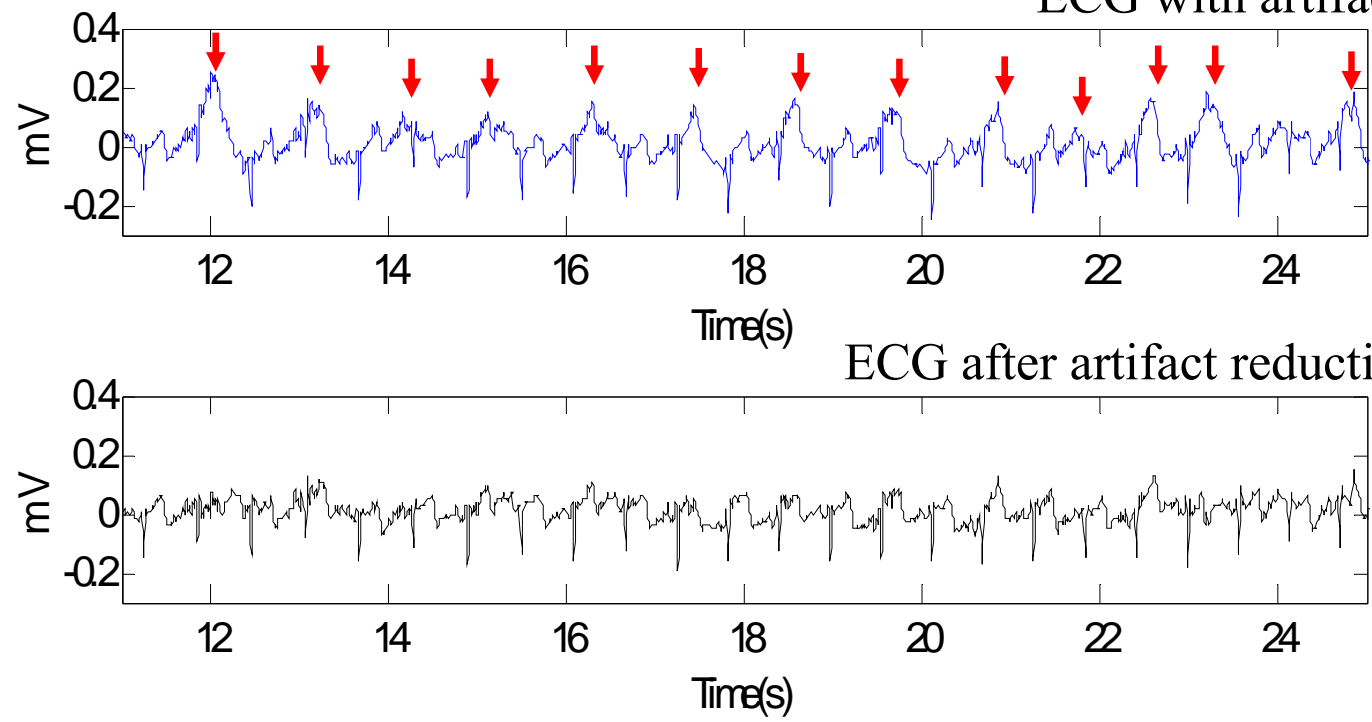

$\downarrow$ Artifacts 
Figure 36. Ambulatory ECG motion artifact reduction (recording 7)

The adaptive filter won't add "wrong" data to the corrupted ECG. Since it takes some time for LMS filters to converge, during this period artifacts may not be greatly reduced. Take Figure 35 as an example: the first four cardiac cycles are artifact-free, and the estimated artifacts are zero for this period of time. The first artifact was generated between 4-5 second. This artifact wasn't reduced after artifact reduction, as it can still be clearly identified after artifact reduction (trace 4 of Figure 35). The reason is that during this period the LMS filter was in the training process and the filter was trying to "track" the artifacts. After that, the artifacts generated in 530 second have been reduced from ECG signal (trace 4 of Figure 35). Another concern is that the filter may take away some portion of good ECG signals. In Figure 35, the QRS complex amplitudes may be changed after artifact reduction, compared with artifact-free ECG signals. But the general morphologies of QRS complex and T waves are kept or recovered after artifact reduction. For P waves, their amplitudes are so small that they can be easily buried in the existing noise generated by muscle contractions. The high frequency muscle contraction induced noise can be reduced by a high pass filter.

Figure 37 shows the LMS filter weights change over time. Since there is no prior knowledge about the statistics of the true artifacts, all the initial value of the weights were set as zero, which is a general procedure in signal processing. In the first a few seconds of this recording, there were no motion artifacts generated. The weights in the first a few seconds are kept as zero. As time goes by, the filter 
automatically adjusts its weights, which makes adaptive filter different from a fixed filter. The coefficients of a fixed filter won't change over time. The results demonstrated that the skin stretch induced motion artifacts have dynamic statistical characteristics.

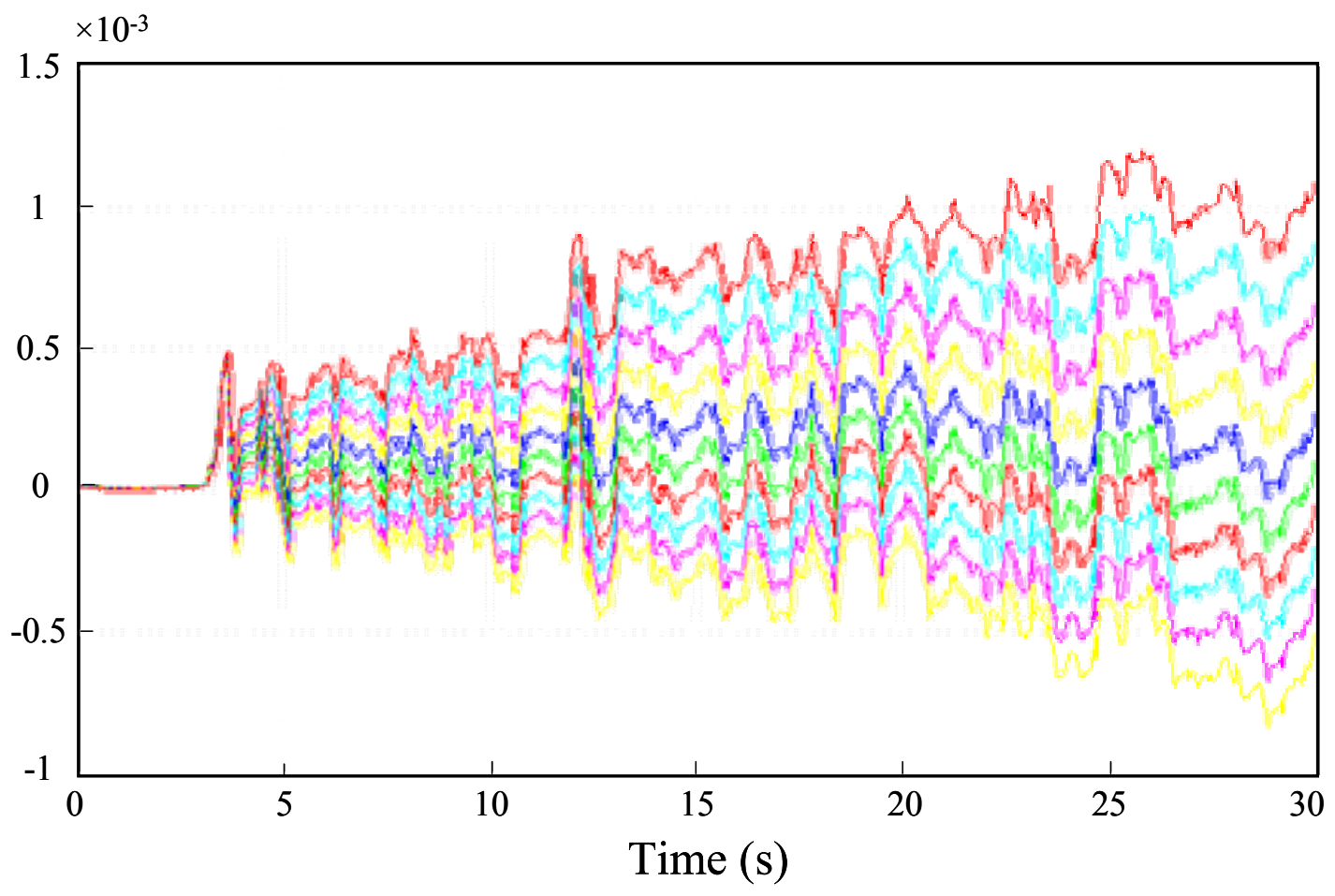

Figure 37. LMS filter weights

The sensitivity of the filter performance was analyzed by changing the filter order and step-size parameter. The results were shown in Figure 38. For the same filter step-size parameter, the larger the filter order $\mathrm{M}$ is, the better the artifact reduction performance. The artifact reduction can improve 2-5\% more when increasing $\mathrm{M}$ by 1 . For the same filter order, the larger the step size parameter, the more the artifact reduction. There is a maximum value for the step size parameter. 
Beyond this maximum value will cause the filter fail to reduce artifacts. This upper boundary can be defined by $\mu<2 /\left(\mathrm{MS}_{\max }\right)$, where $\mathrm{S}_{\max }$ is the maximum value of the power spectral density of $u(n)$.

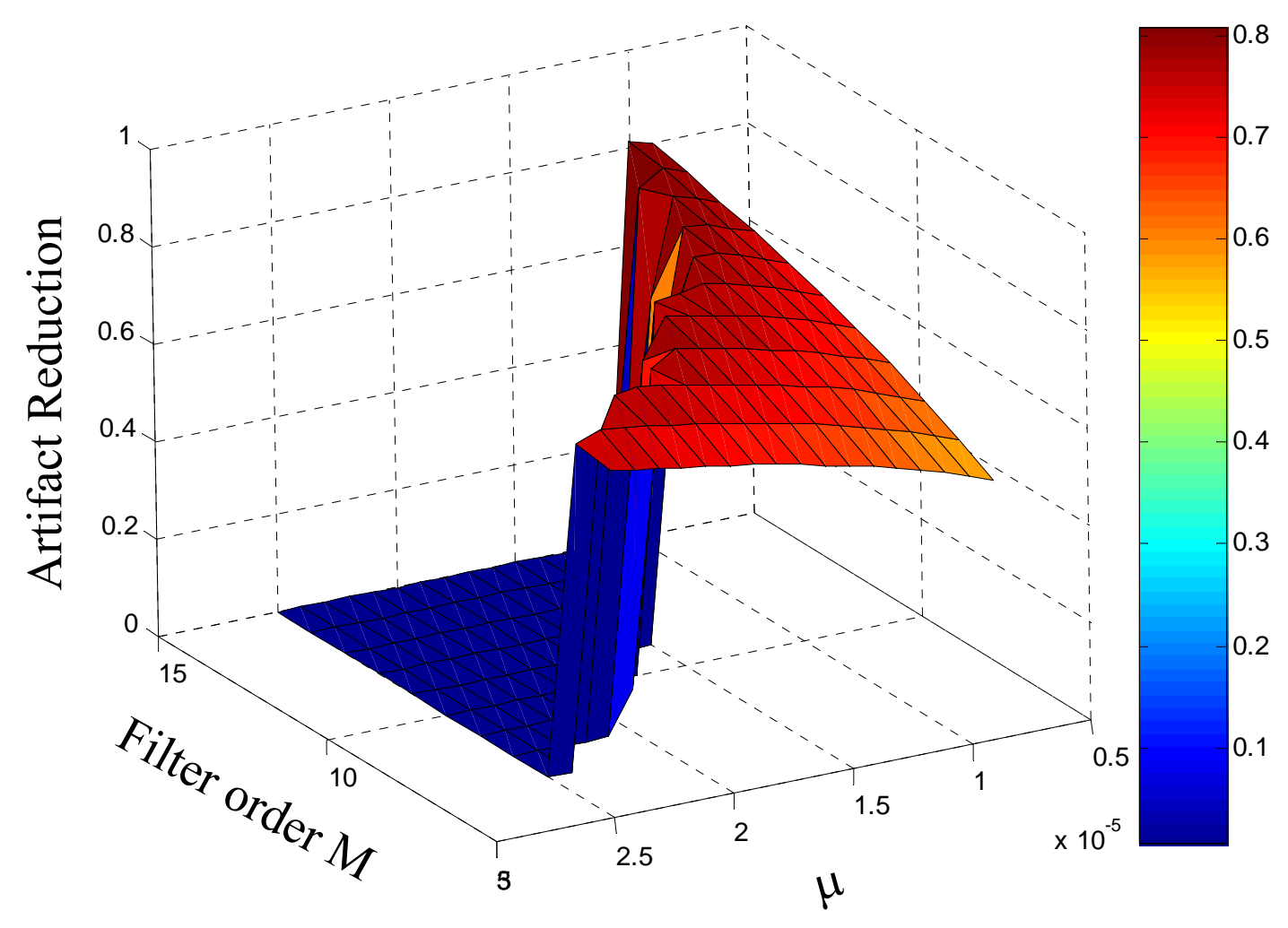

(a)

Figure 38. LMS filter performance

For each filter order $\mathrm{M}$, there is a maximum noise reduction percentage when changing the step size parameter. Increasing the filter order slightly improves the filter performance, as shown in Figure 39. Mathematically, this can be explained that the current value of skin strain plays a more important role in artifacts estimation than previous data. In other words, the characteristic of the skin induced artifacts varies 
quite fast so that history of the skin strain signal is not as important as the current value.

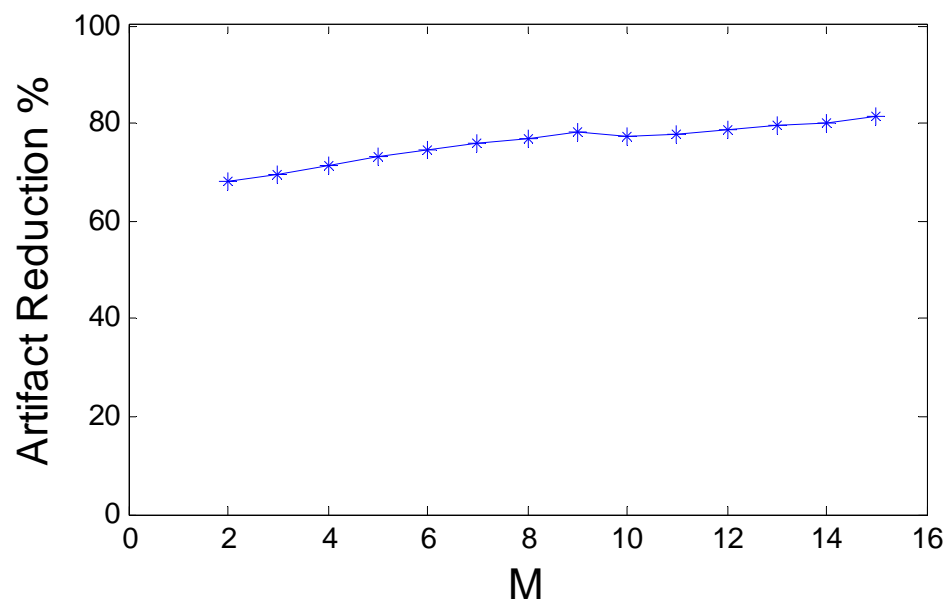

Figure 39. LMS filter performance vs. filter order

Artifact reduction performance varies in different types of motion. This is because the optical sensor is easier to go out of focus during motions that induce more vertical movements of the sensor. Time frame 10-12 second of Figure 34 was zoomed in Figure 40 (a). An artifact exists between 10-10.5 second (trace 3 of Figure 40 (a)), and hasn't been reduced after going through artifact reduction (trace 4 of Figure 40 (a)). This artifact has a peak amplitude of $0.36 \mathrm{mV}$, which is much larger than the $\mathrm{T}$ wave peak amplitude $(0.06 \mathrm{mV})$. Trace 4 of Figure 40 (a) indicates that the skin strain signal was failed to be picked up by the optical sensor (A captured skin strain will result in optical sensor output to be approximately 50 ). On the other hand, in the top trace of Figure 35, the optical sensor captured all the skin strain signal while the subject was raising the arm. 
Residual motion artifacts can also be due to the characteristic change of skin electrophysiology during stretching. Thus there is no constant correlation between skin stretch detected by optical sensor and the amplitude of the ECG artifacts. The adaptive filter has to keep finding converging solutions in this dynamic environment. Time frame 20-26 seconds of Figure 34 was zoomed in in Figure 40 (b). Between 24 and 25 seconds, skin stretch was in similar amounts of amplitudes as those between 23 and 24 seconds, while artifacts were larger. If the adaptive filter changed its solution according to the dynamic changes in inputs before the artifacts were fully picked, residual artifacts were left.

During the experiments, it was observed that stretching the skin did not always induce ECG artifacts. In other words, a subject's skin stretch induced artifacts may be larger than the ECG signal for a certain period of time, and then disappear over another time frame. The reason hasn't been clearly reported in the literature, although. Odman (1981) observed that time sequences for the skin stretch potentials vary individually.

Up to now there is no widely accepted explanation on why skin strain induces skin voltage change. Since adaptive filters captures the dynamic relationship between skin strain signal and ECG artifacts, the phenomena discussed above is not a concern. For the time period when stretching skin causes no or little artifacts, the filter weights are quickly adjusted to zero or close to zero, indicating that artifacts are negligible in this time frame. 

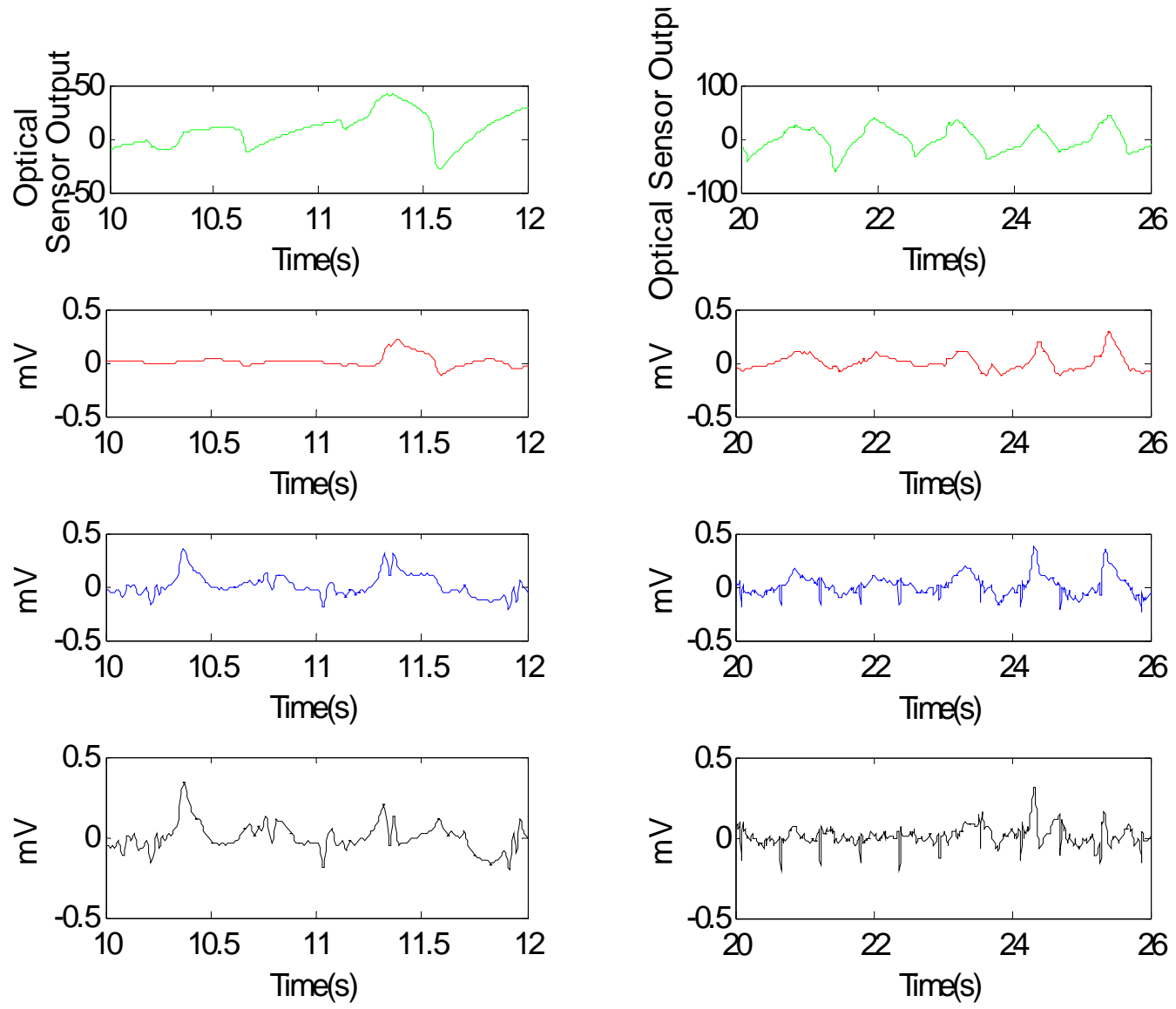

(a)

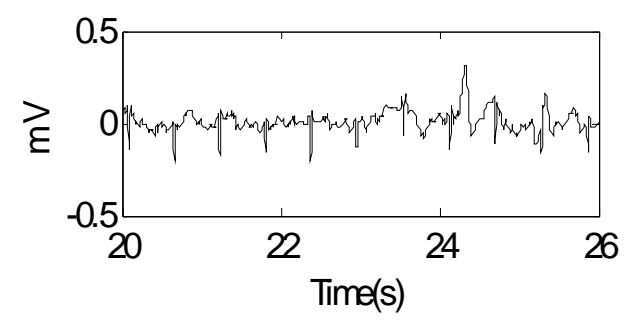

(b)

Figure 40. Ambulatory ECG motion artifact reduction. ECG was recorded when the subject is slightly stretching the upper chest (recording 1). First trace: skin strain signal; second trace: estimated artifacts; third trace: ECG with artifacts; fourth trace: ECG after artifact reduction

The artifact reduced ECG can be used in applications such as heart rate measurement and arrhythmia detection (Thakor and Zhu 1991). In some cardiac cycles, artifacts reduction recovered ECG morphology. Figure 41 shows that in artifact corrupted ECG, T waves were buried in artifacts: artifacts last approximately 0.6 second and have a peak value of $0.2 \mathrm{mV}$, while $\mathrm{T}$ wave only last about 
approximately 0.2 seconds, and have a peak value of $0.06 \mathrm{mV}$. ECG after artifact reduction has similar morphology to that of the artifact-free ECG, which indicates a good recovery.
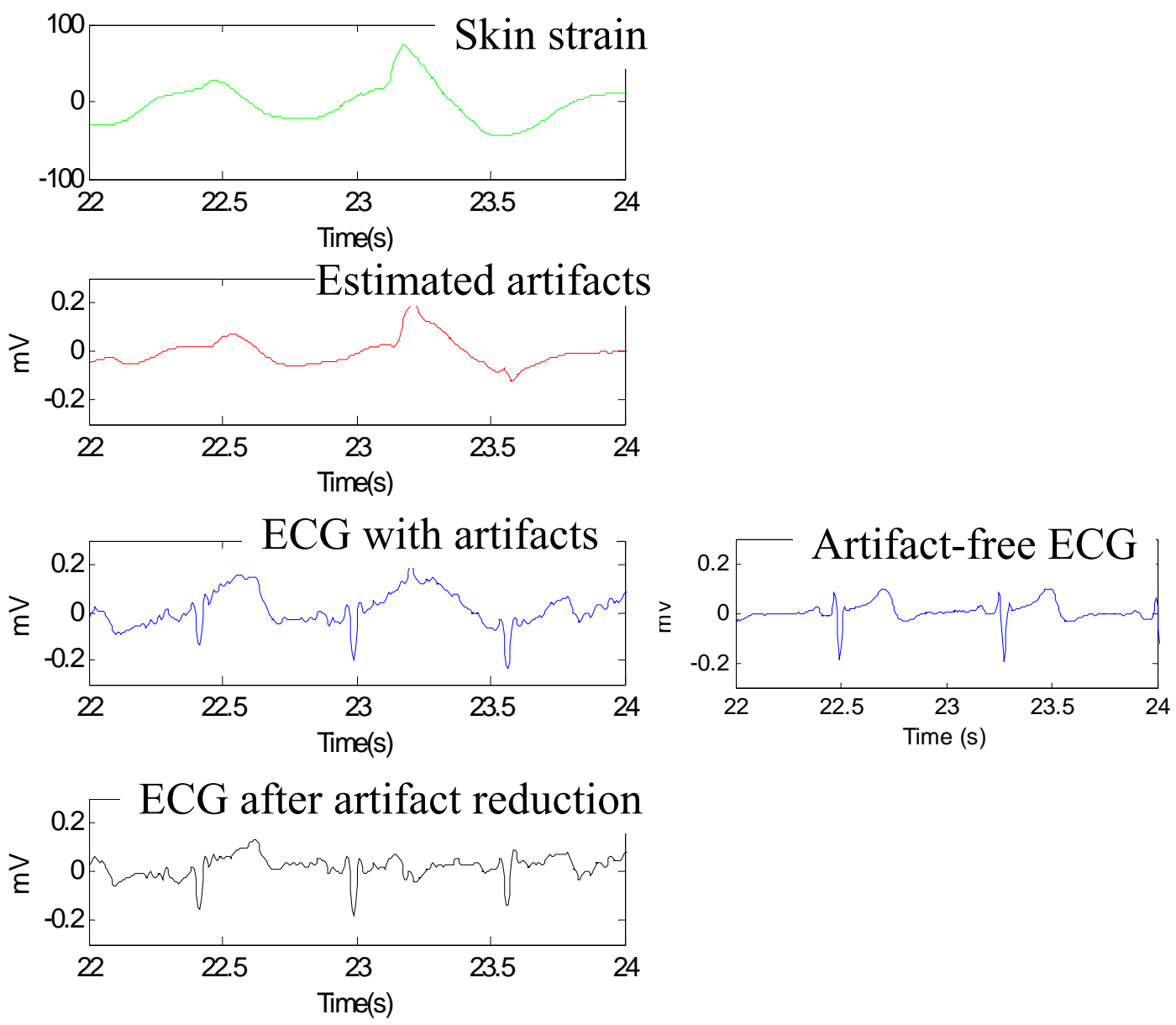

Figure 41. Ambulatory ECG motion artifact reduction (recording 6: subject is raising the left arm)

Artifact reduction performance can also be analyzed in frequency domain. Fast Fourier transform was conducted for ECG signals before and after artifact reduction (second and third trace of Figure 42). Frequency components of artifact free ECG was the reference (top trace of Figure 42). The majority of frequency 
components of skin stretch induced artifacts are below $2 \mathrm{~Hz}$, which overlaps with ECG frequency $(1.7 \mathrm{~Hz}$ peak), as seen in trace two of Figure 42. After artifact reduction, this part was largely reduced, as can be seen in the third trace of Figure 42. Note that since artifact-free ECG and ECG with artifacts were not recorded simultaneously, the heart rates when the two signals were recorded were slightly different. This explains that the frequency components shifted $0.3-0.5 \mathrm{~Hz}$ between the top trace and the bottom two traces.
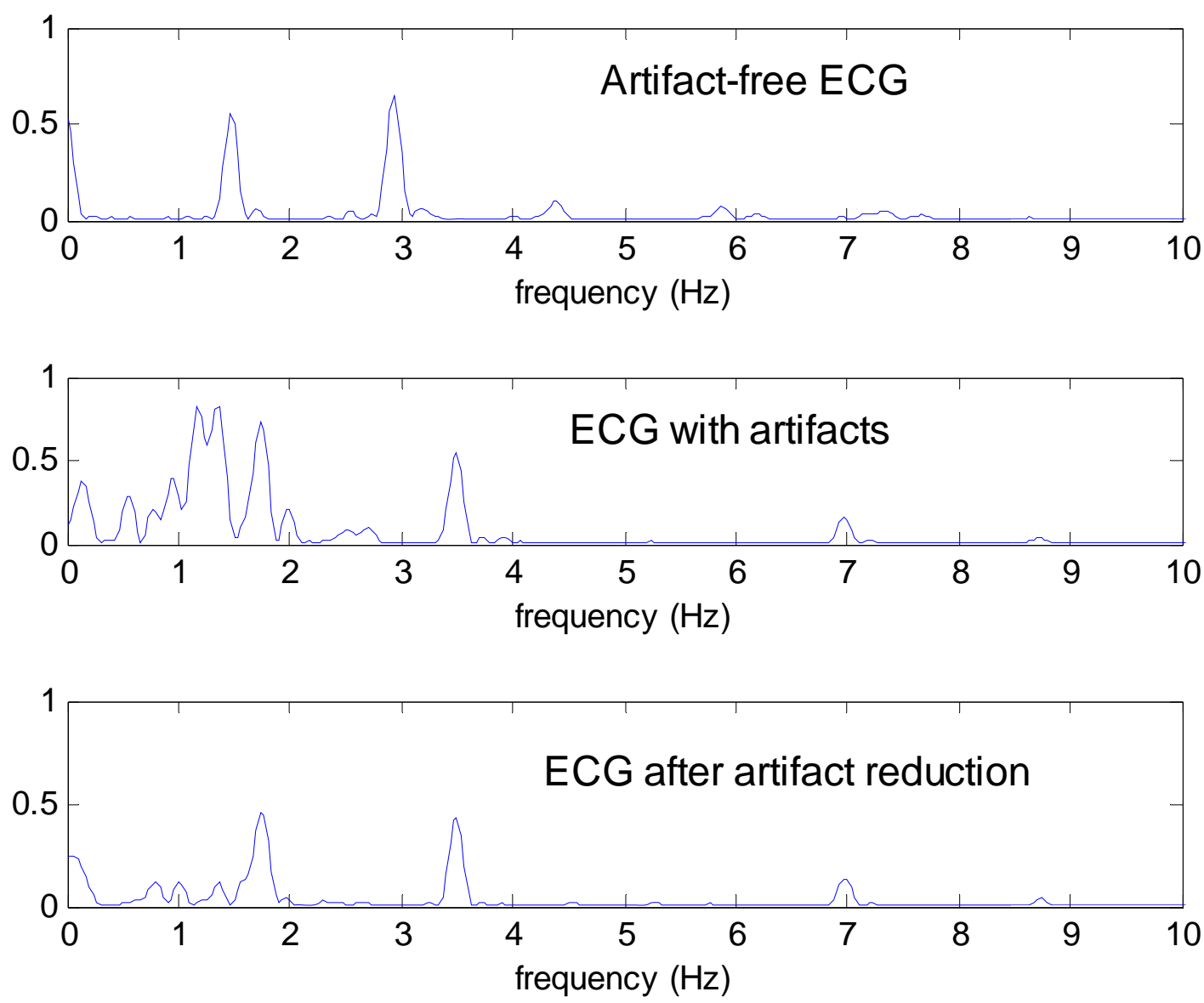

Figure 42. Frequency domain analysis of artifact-free ECG (first trace), ECG with artifacts (second trace), and ECG after artifact reduction (third trace). The top 
trace is recorded when the subject was at rest. The second and third traces were taken when the subject was raising the left arm.

\section{$\underline{5.6 \text { Conclusions }}$}

A wearable sensor system to non-invasively reduce ECG motion (skin-stretch) artifacts with an optical sensing technique and adaptive filtering has been developed and demonstrated. Calibration results demonstrated that the optical sensor can be used as a non-invasive wearable skin strain sensor with high linearity $\left(\mathrm{R}^{2}=0.98\right)$.

ECG is widely used in standard clinical practice. During stress test, ECG is recorded while the patient is moving (walking, running) on a treadmill. The purpose of this test is to diagnose coronary artery disease, determine the cause of chest pain and exercise capacity of the heart, and to identify rhythm disturbances. The heart rate and morphologies of the ECG waveforms are important for diagnosis. Ambulatory ECG monitoring allows a patient to wear a recorder and continuously record ECG for 24 to 48 hours; or for a few months, and trigger the ECG recording upon symptoms. The purpose of ambulatory ECG recording is to detect infrequent arrhythmia which may not be detected in a doctor's office. Significant features including the ECG waves' individual durations, and time intervals including the P-R, S-T and Q-T intervals are analyzed offline for arrhythmia diagnosis. Other than that, for patients whose heart beats needs to be continuously monitored during their hospital stay. Accurate detection of heart rate and rhythm is necessary, and false alarms can be induced by motion artifacts. The sensor system and method can be applied in these 
applications for artifact reduction. In addition, the system and method can be further applied to motion artifact reduction of other biopotential recordings.

Experimental results demonstrated that this sensor system and method can effectively remove skin strain induced artifacts from ECG signals. On average, this wearable sensor system and method can reduce $85 \%$ of motion artifacts under various types of ambulatory conditions, such as walking, raising the arm, and running. Increasing the filter order slightly improves the filter performance. Even in the worst case, the method can achieve artifact reduction by $43 \%$, which was caused by non-flat skin / body surface, vertical deformation of the skin, or vertical movement of the sensor that may cause the image to lose focus. Signal stability of the optical sensor output is currently the key challenge of this system and method. Device miniaturization is suggested to ensure a proper sensor attachment to the skin surface during motions.

Correctly measuring skin stretch in-vivo is a complicated task. Using the optical sensor to detect skin strain can be applied in other applications, as currently there is no successful method to accurately measure soft tissue strain in a wearable manner. Examples include research on skin mechanical properties for dermatologists and biologists in medicine, evaluation of skin disease and aging, and skin deformation assessment. The optical strain sensor can be used as a noninvasive, simple and cost effective skin / soft tissue strain sensor. Other advantages include high resolution, high sampling rate, and the potential to be used to measure local skin strain in real time during ambulatory conditions. 


\section{Chapter 6: Contributions}

The contributions from this thesis are:

- Identified the limitations in existing and emerging non-invasive ambulatory cardiovascular monitoring techniques to improve the device design and wearability

- Developed a method that utilizes measured skin strain signal as a reference input in adaptive filtering to reduce ECG motion artifacts

- Demonstrated the feasibility of using an optical technique as a wearable skin strain sensor for non-invasive ambulatory monitoring

- Demonstrated that the system and method that utilizes optical sensing and adaptive filtering technique can reduce skin stretch induced artifacts

- Demonstrated that the wearable system and method can reduce motion artifacts in daily activities for ambulatory ECG monitoring

- Applied for patent "Sensor Systems and Methods for Reducing Motion Artifact" 


\section{Thesis Publications}

\section{Appendices}

- Liu, Y. and Pecht, M.G., "Reduction of Motion Artifacts in Electrocardiogram Monitoring", to be submitted.

- Liu, Y. and Pecht, M., "Reduction of Skin Stretch Induced Motion Artifacts in Electrocardiogram Monitoring Using Adaptive Filtering”, 28th International Conference of the IEEE Engineering in Medicine and Biology Society: Engineering Revolution In BioMedicine, New York, NY, August 31-September 3, 2006.

- Eveloy, V., Liu, Y., Pecht, M., "Developments in Ambulatory Electrocardiography", Biomedical Instrumentation and Technology, Vol. 40, No. 3, pp 238-245, May/June, 2006.

- Liu, Y., Eveloy, V. and Pecht, M., "Non-invasive Electrocardiogram and Blood Pressure Monitors for Cardiovascular Disease", 38th International Symposium on Microelectronics, Sensor and MEMS Packaging, pp. 66-74, Philadelphia, PA, September 25-29, 2005.

- Eveloy, E., Liu, Y., and Pecht, M., "Heart Sound Measurement and Analysis in Cardiovascular Disease Assessment", Surface Mount Technology Association (SMTA), Proceedings of the SMTA Medical Electronics Symposium, Minneapolis, MN, April 25-27, 2005.

- Pecht, M. and Liu, Y., "Sensor Systems and Methods for Reducing Motion Artifact", reported to the Office of Technology Commercialization, University of Maryland, December, 2005, Ref. No. PS-2005-110. 


\section{5 ambulatory ECG recordings before and after artifact reduction}

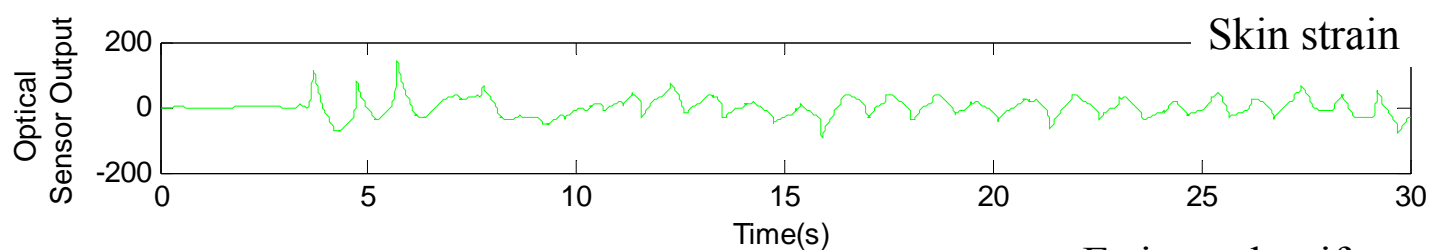

Estimated artifacts
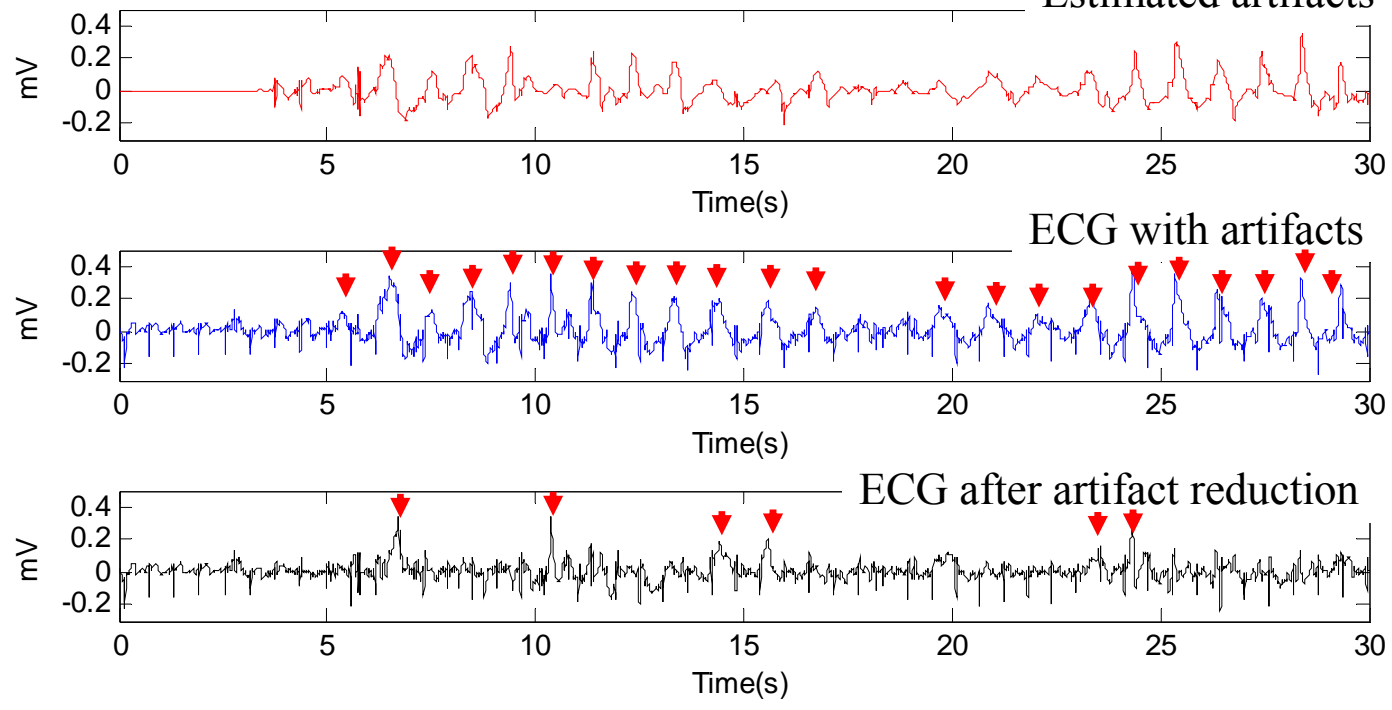

Ambulatory ECG motion artifact reduction (recording 1: subject is slightly stretching the upper chest); top trace: optical sensor output; second trace: artifacts estimated by the adaptive filter; third trace: ECG signal corrupted by artifacts; bottom trace: ECG signal after artifact reduction (Figure 34) 

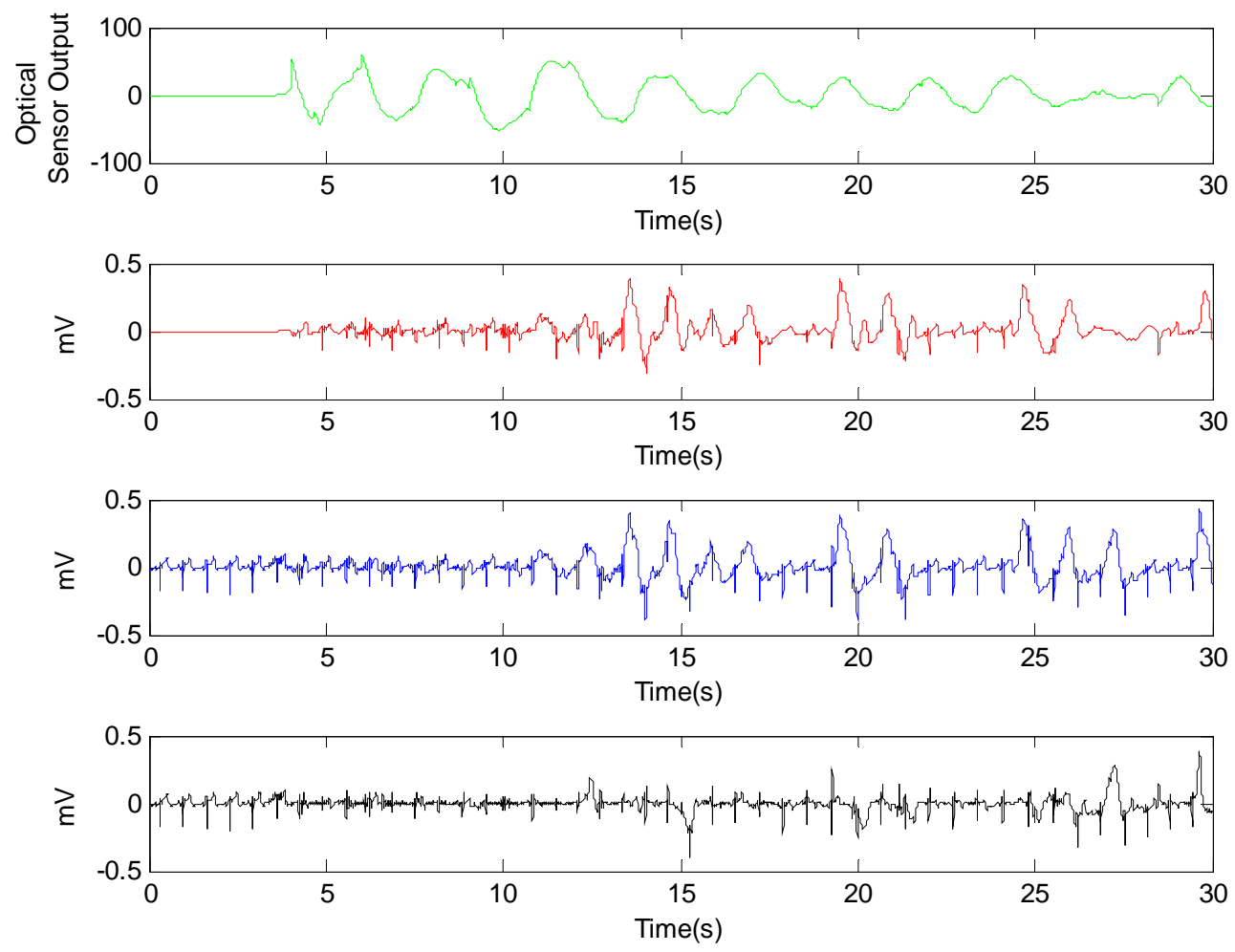

Ambulatory ECG motion artifact reduction (recording 2: subject is slightly stretching the upper chest); top trace: optical sensor output; second trace: artifacts estimated by the adaptive filter; third trace: ECG signal corrupted by artifacts; bottom trace: ECG signal after artifact reduction 

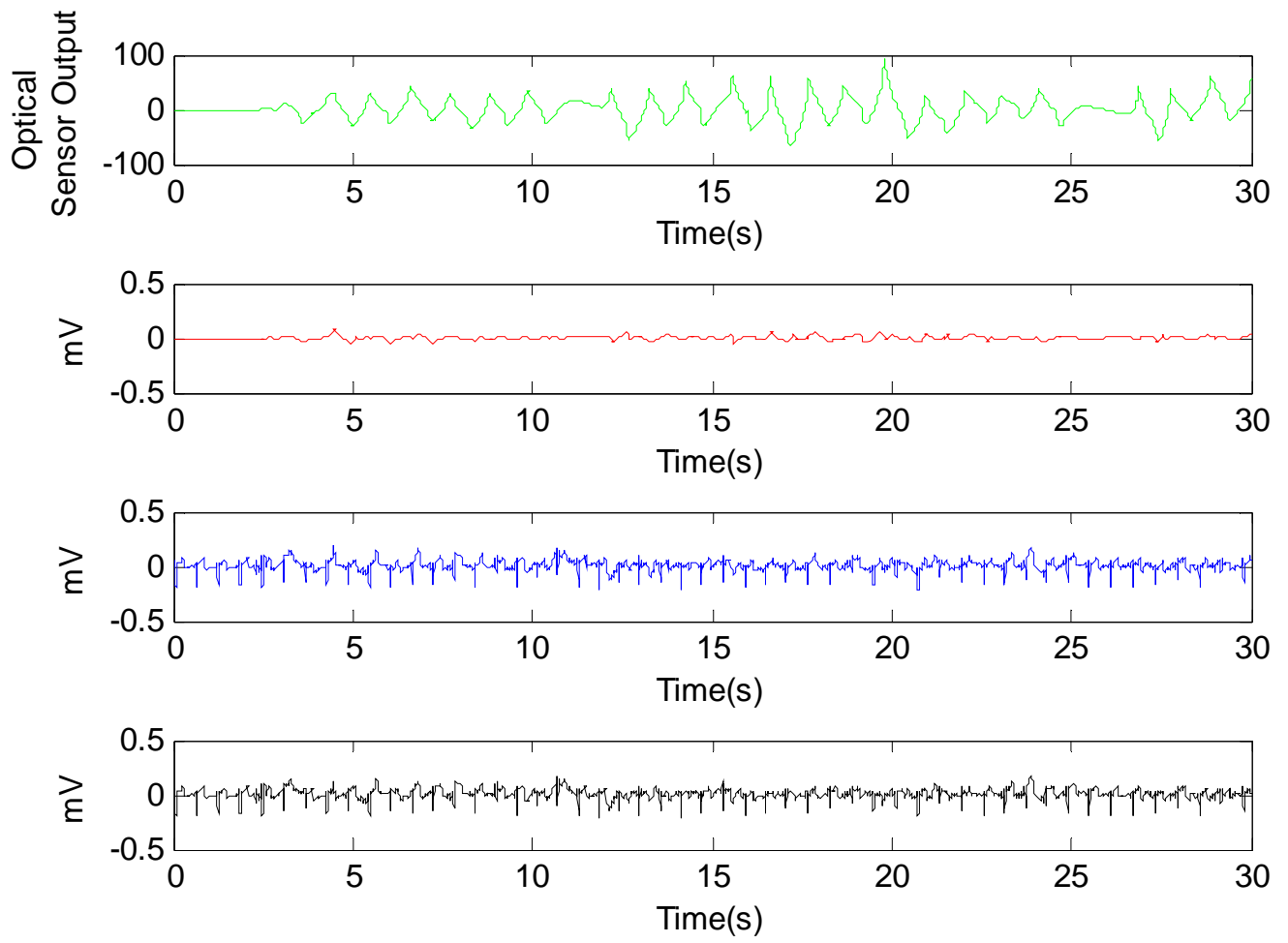

Ambulatory ECG motion artifact reduction (recording 3: subject is horizontally waving the left arm); top trace: optical sensor output; second trace: artifacts estimated by the adaptive filter; third trace: ECG signal corrupted by artifacts; bottom trace: ECG signal after artifact reduction 

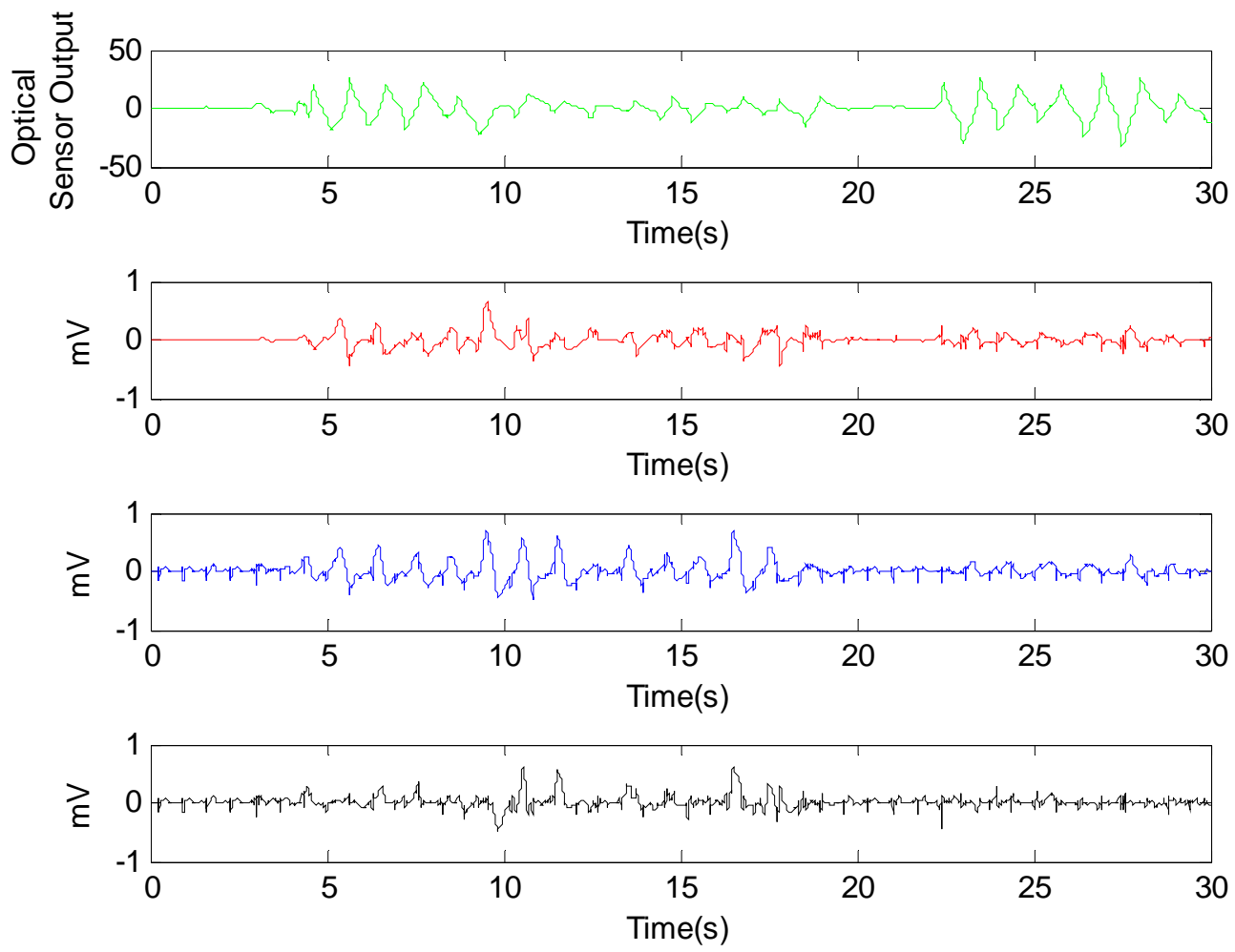

Ambulatory ECG motion artifact reduction (recording 4: subject is horizontally waving the left arm); top trace: optical sensor output; second trace: artifacts estimated by the adaptive filter; third trace: ECG signal corrupted by artifacts; bottom trace: ECG signal after artifact reduction 

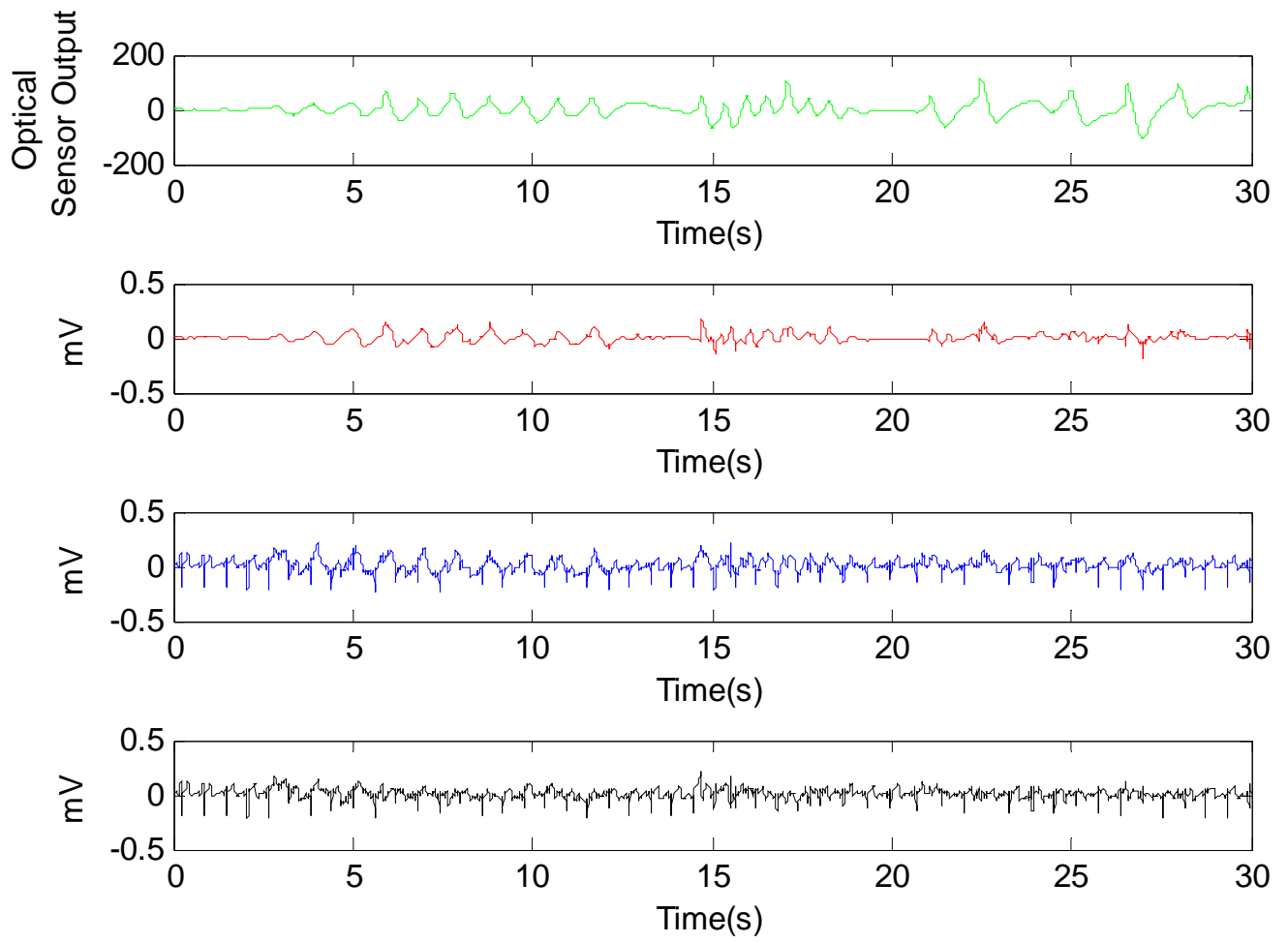

Ambulatory ECG motion artifact reduction (recording 5: subject is horizontally waving the left arm); top trace: optical sensor output; second trace: artifacts estimated by the adaptive filter; third trace: ECG signal corrupted by artifacts; bottom trace: ECG signal after artifact reduction 

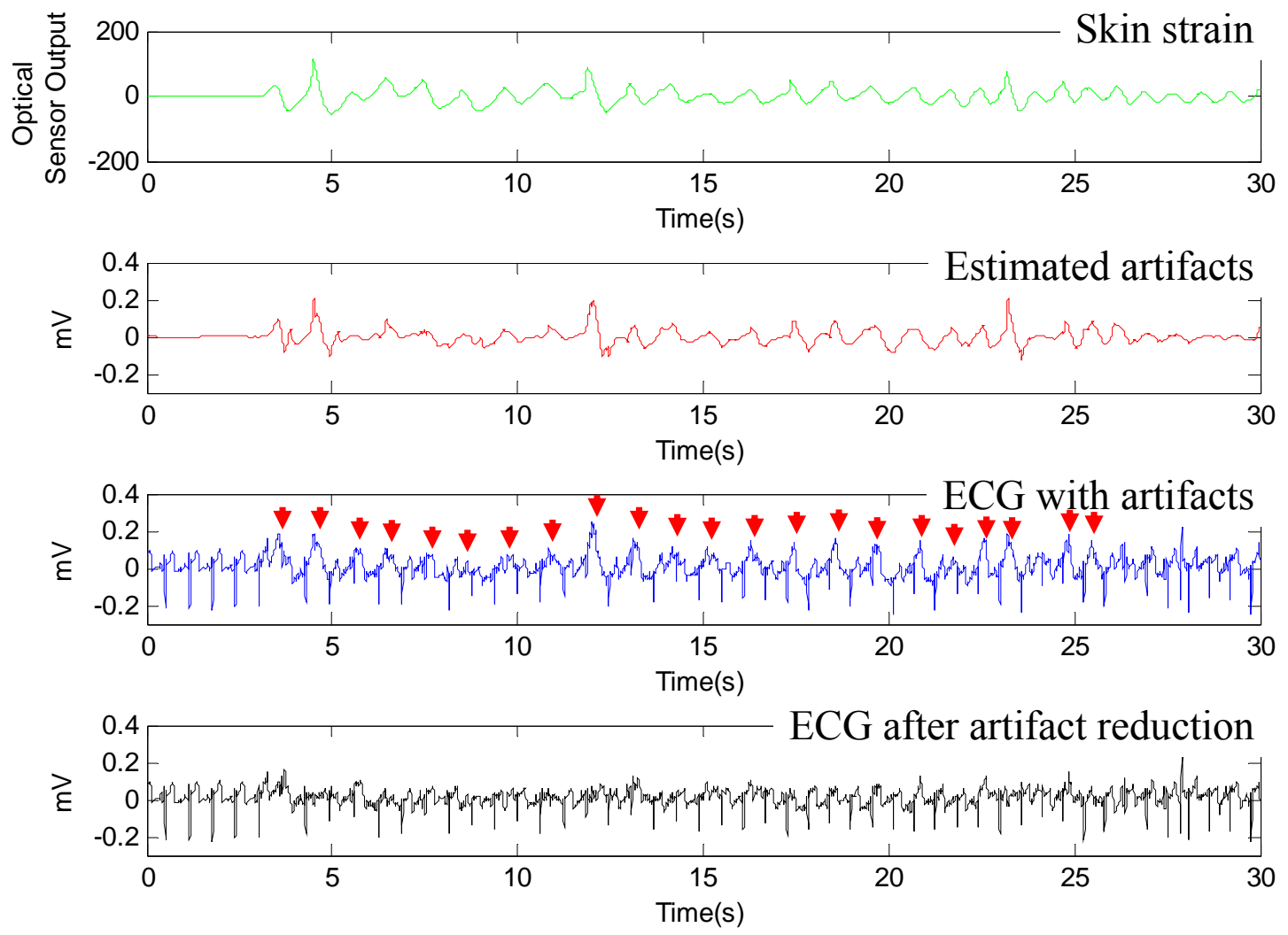

Ambulatory ECG motion artifact reduction (recording 6: subject is raising the left arm); top trace: optical sensor output; second trace: artifacts estimated by the adaptive filter; third trace: ECG signal corrupted by artifacts; bottom trace: ECG signal after artifact reduction (Figure 35) 

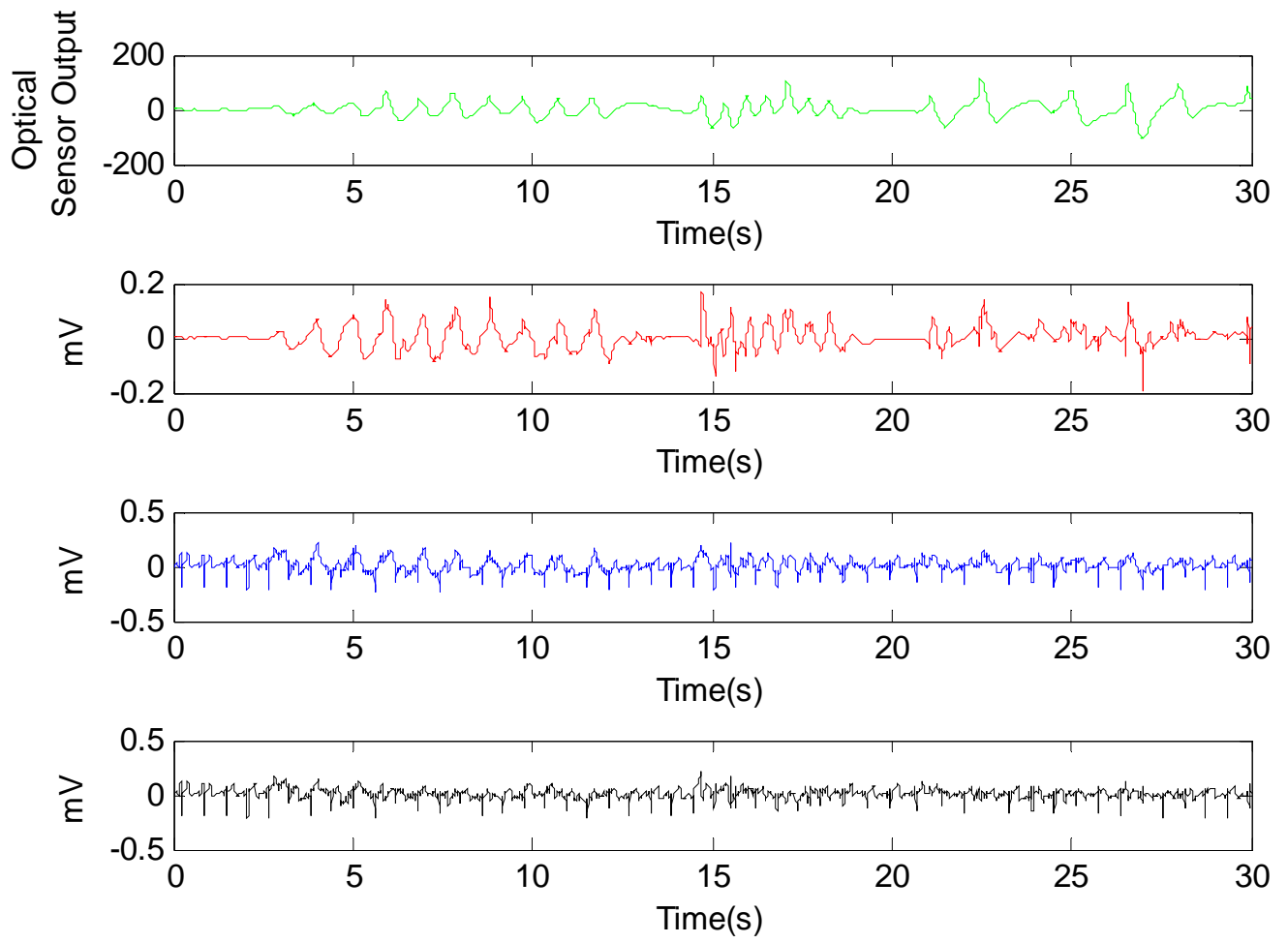

Ambulatory ECG motion artifact reduction (recording 7: subject is raising the left arm); top trace: optical sensor output; second trace: artifacts estimated by the adaptive filter; third trace: ECG signal corrupted by artifacts; bottom trace: ECG signal after artifact reduction 

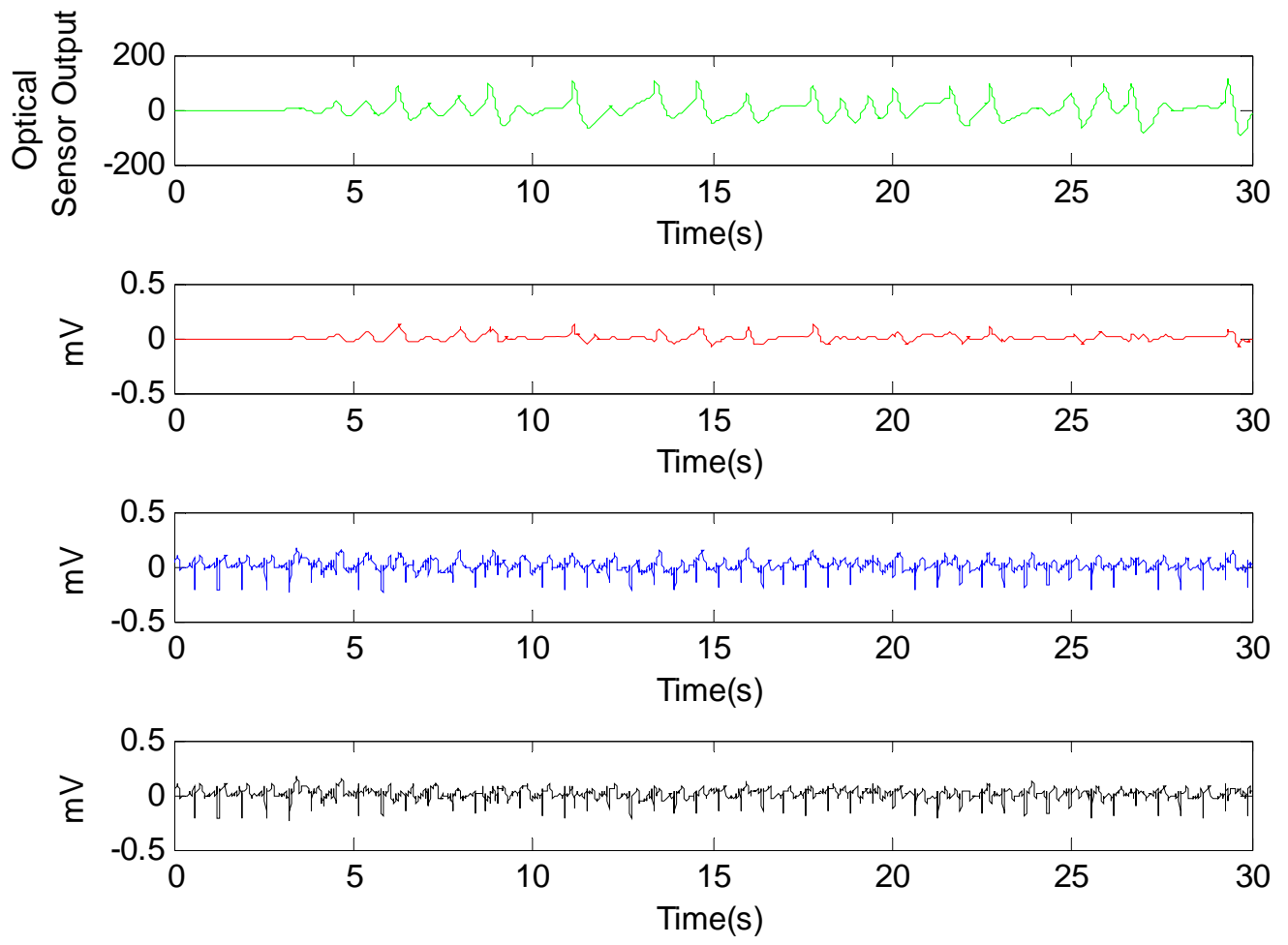

Ambulatory ECG motion artifact reduction (recording 8: subject is raising the left arm); top trace: optical sensor output; second trace: artifacts estimated by the adaptive filter; third trace: ECG signal corrupted by artifacts; bottom trace: ECG signal after artifact reduction 

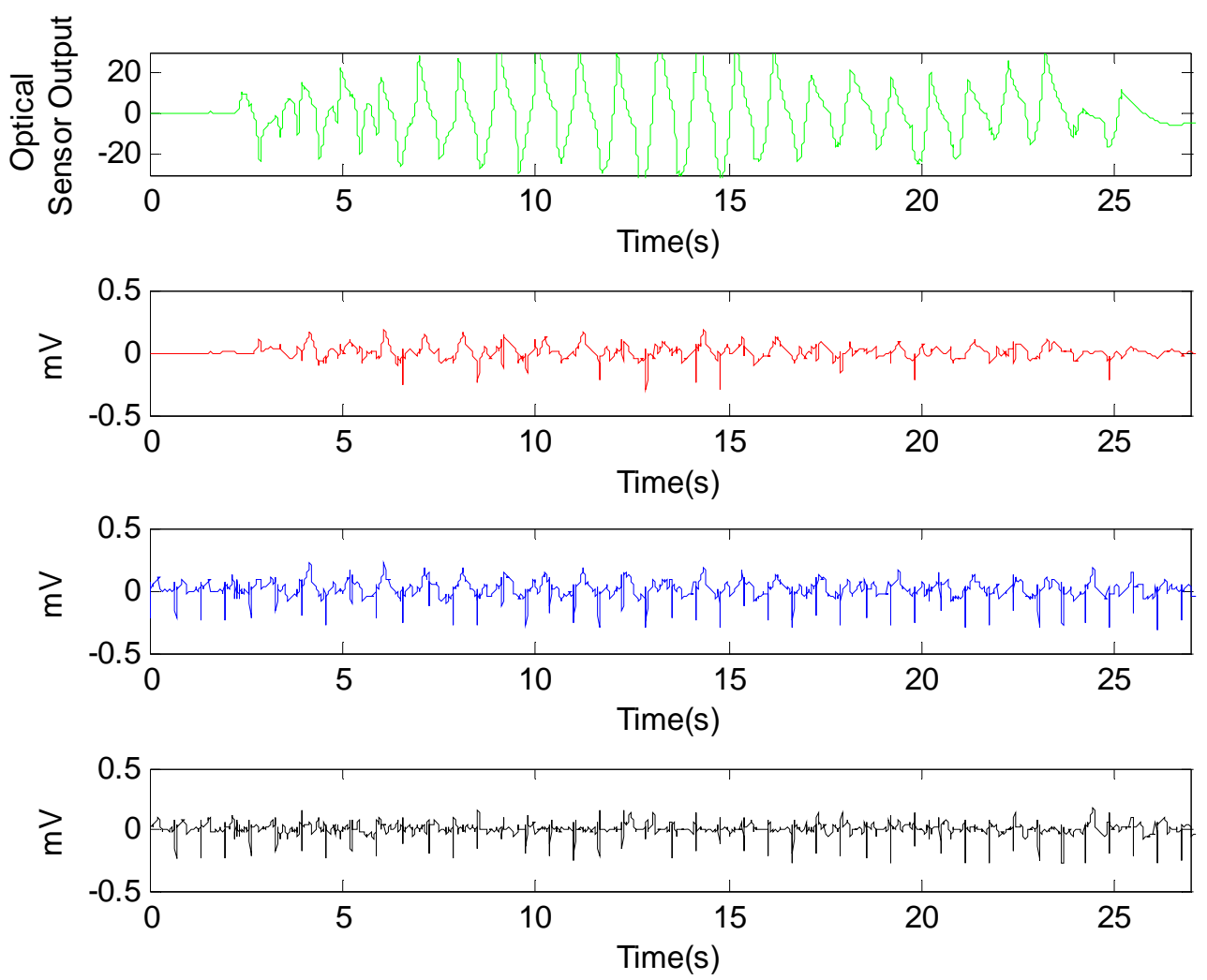

Ambulatory ECG motion artifact reduction (recording 9: subject is walking); top trace: optical sensor output; second trace: artifacts estimated by the adaptive filter; third trace: ECG signal corrupted by artifacts; bottom trace: ECG signal after artifact reduction 

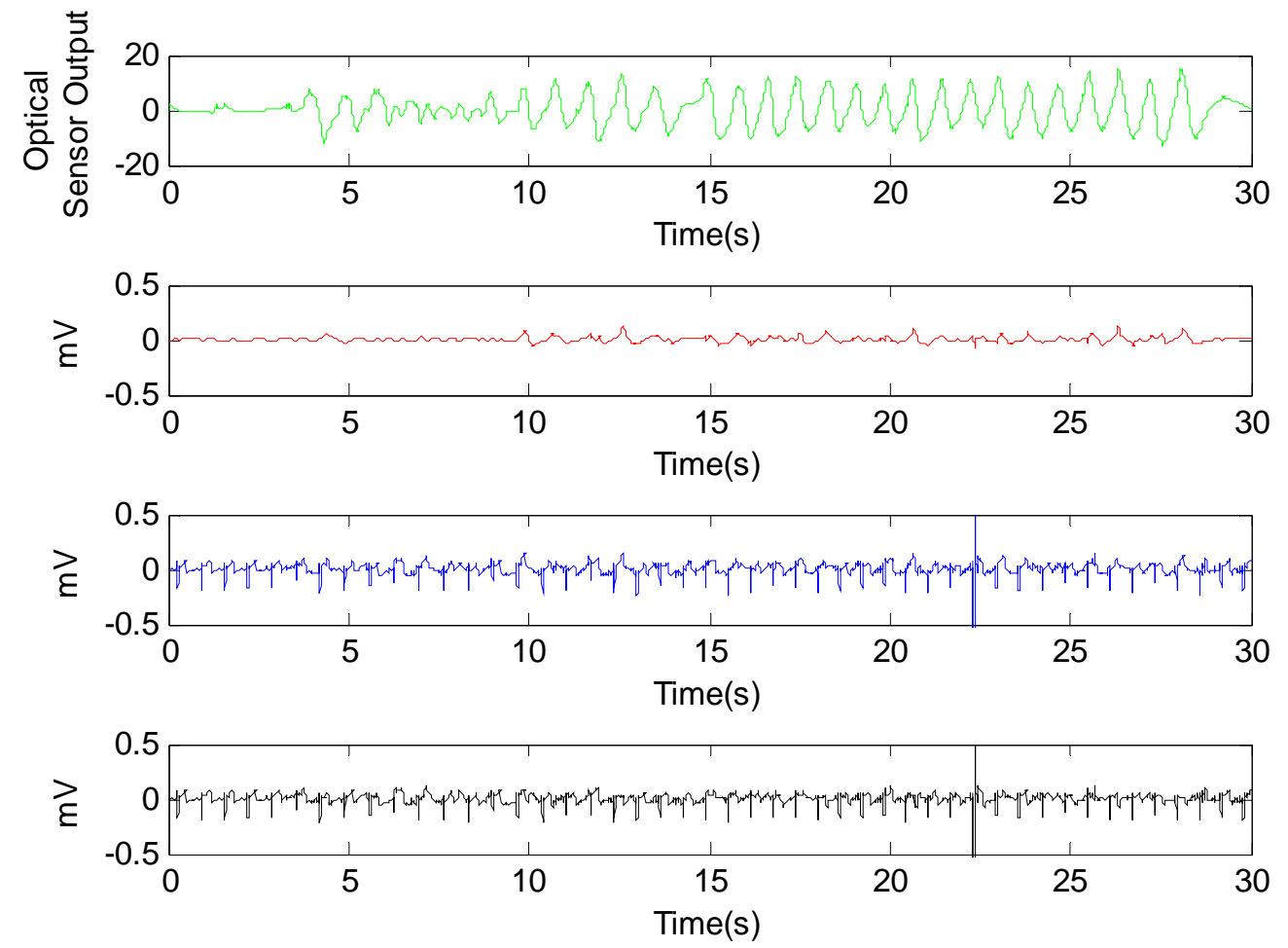

Ambulatory ECG motion artifact reduction (recording 10: subject is walking); top trace: optical sensor output; second trace: artifacts estimated by the adaptive filter; third trace: ECG signal corrupted by artifacts; bottom trace: ECG signal after artifact reduction 

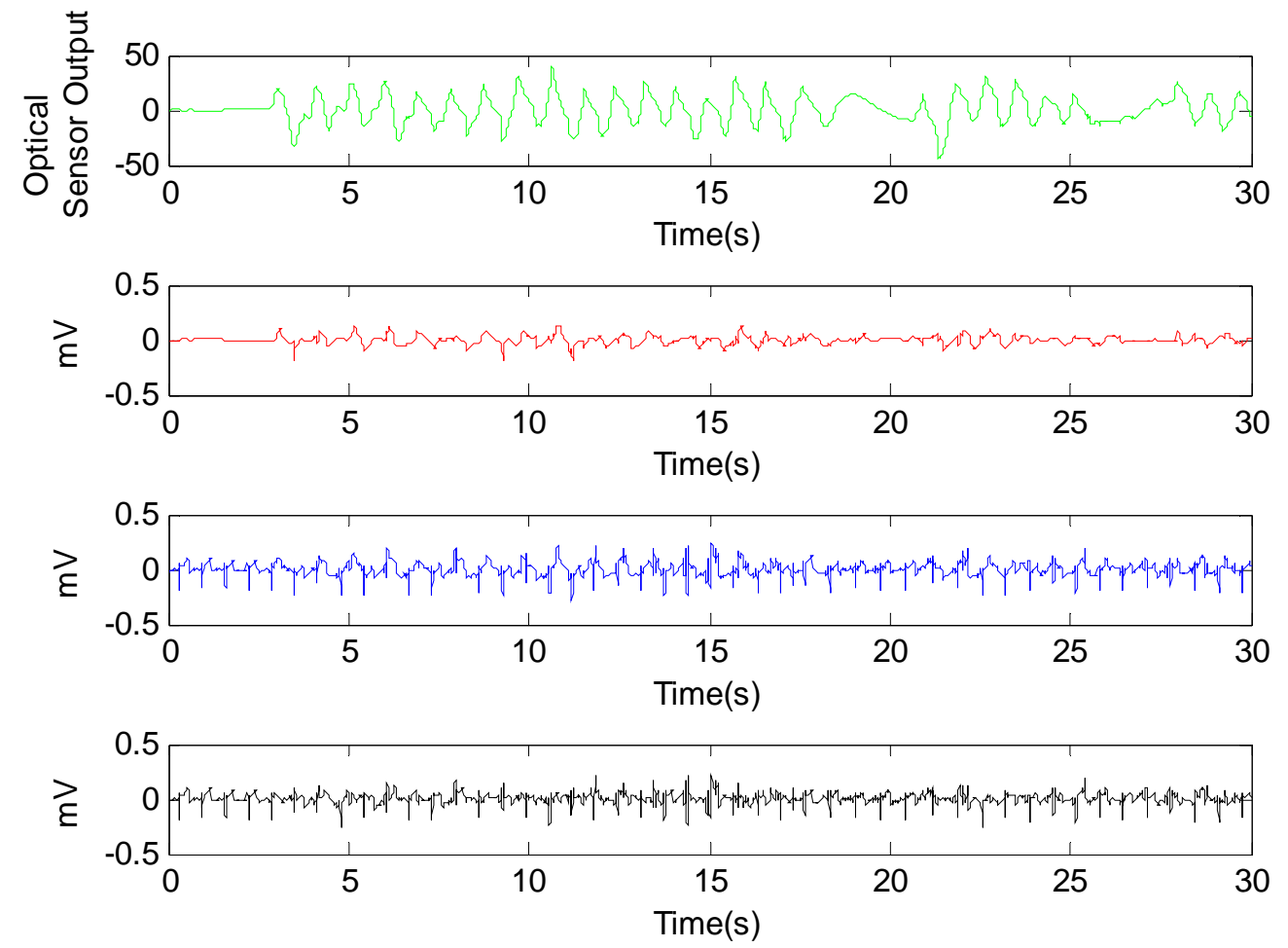

Ambulatory ECG motion artifact reduction (recording 11: subject is walking); top trace: optical sensor output; second trace: artifacts estimated by the adaptive filter; third trace: ECG signal corrupted by artifacts; bottom trace: ECG signal after artifact reduction 

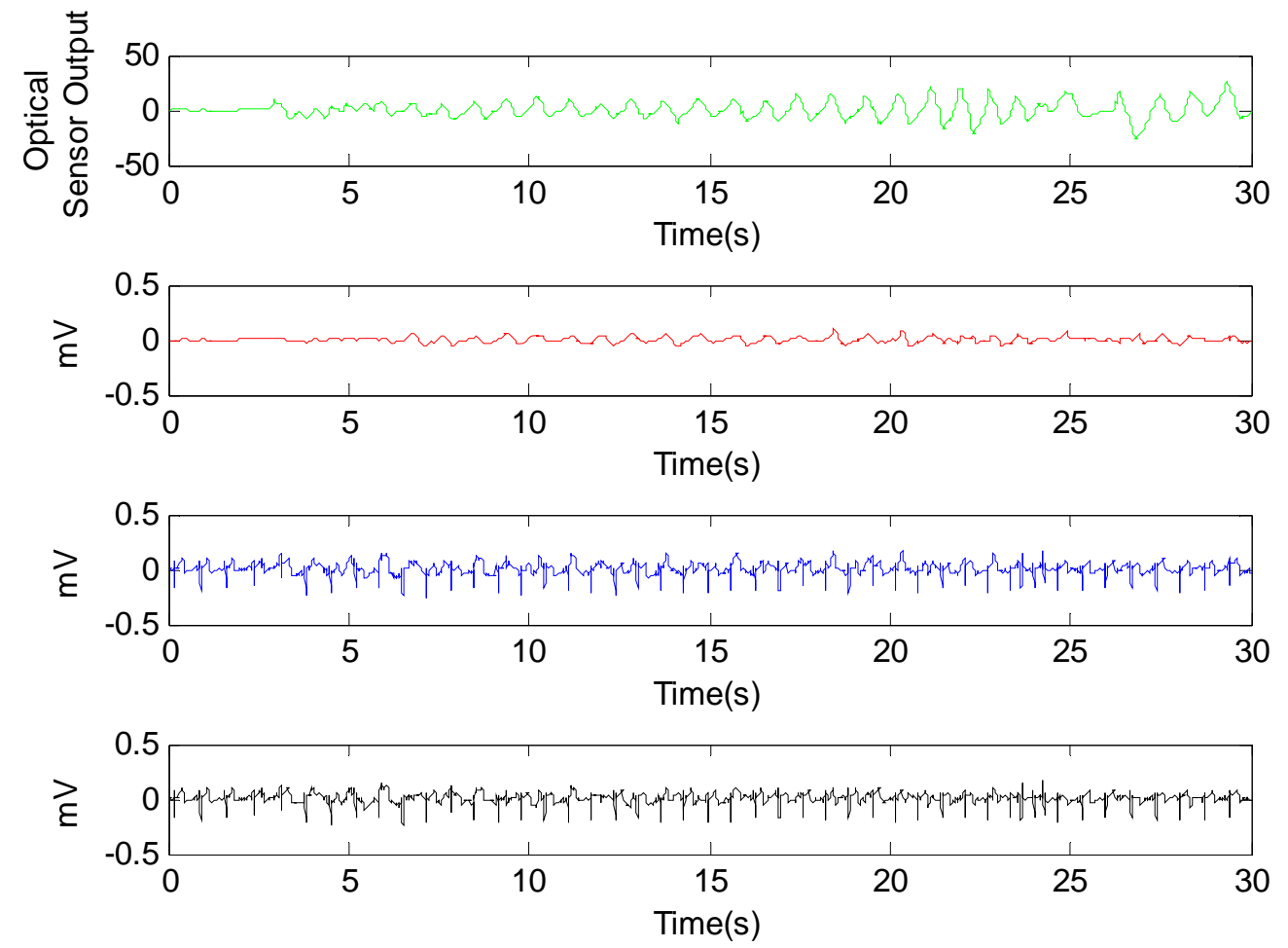

Ambulatory ECG motion artifact reduction (recording 12: subject is walking); top trace: optical sensor output; second trace: artifacts estimated by the adaptive filter; third trace: ECG signal corrupted by artifacts; bottom trace: ECG signal after artifact reduction 

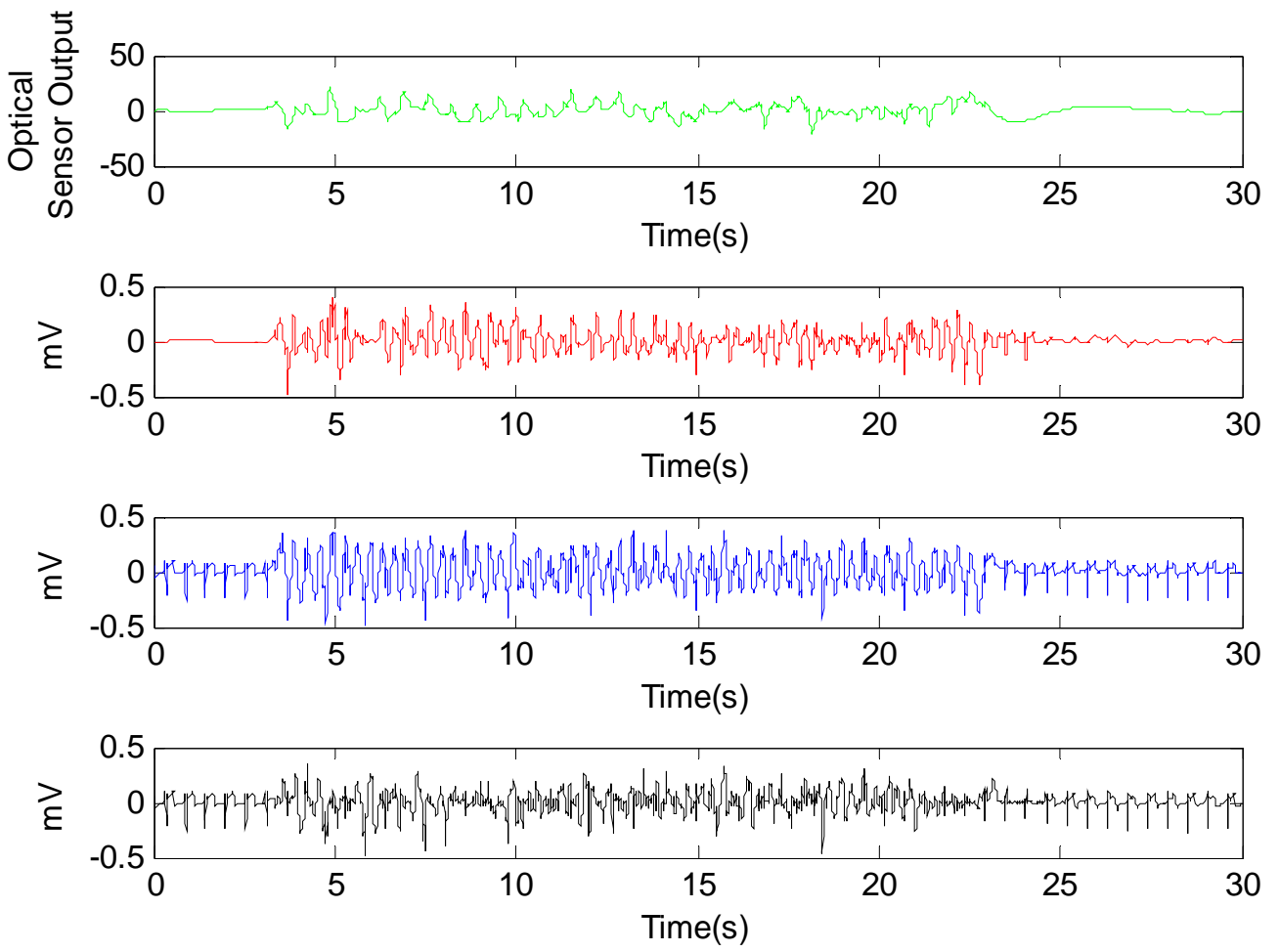

Ambulatory ECG motion artifact reduction (recording 13: subject is running); top trace: optical sensor output; second trace: artifacts estimated by the adaptive filter; third trace: ECG signal corrupted by artifacts; bottom trace: ECG signal after artifact reduction 

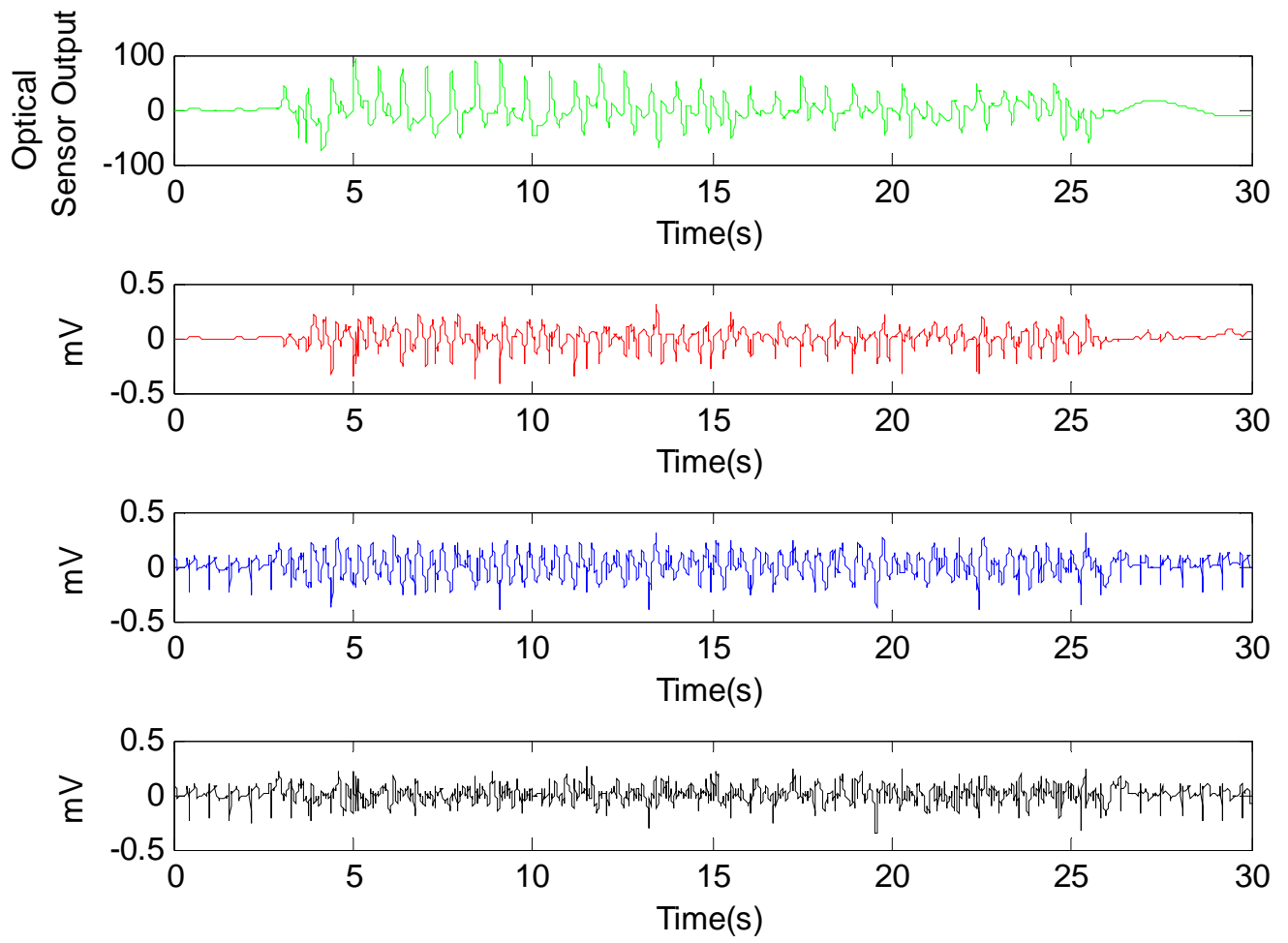

Ambulatory ECG motion artifact reduction (recording 14: subject is running); top trace: optical sensor output; second trace: artifacts estimated by the adaptive filter; third trace: ECG signal corrupted by artifacts; bottom trace: ECG signal after artifact reduction 

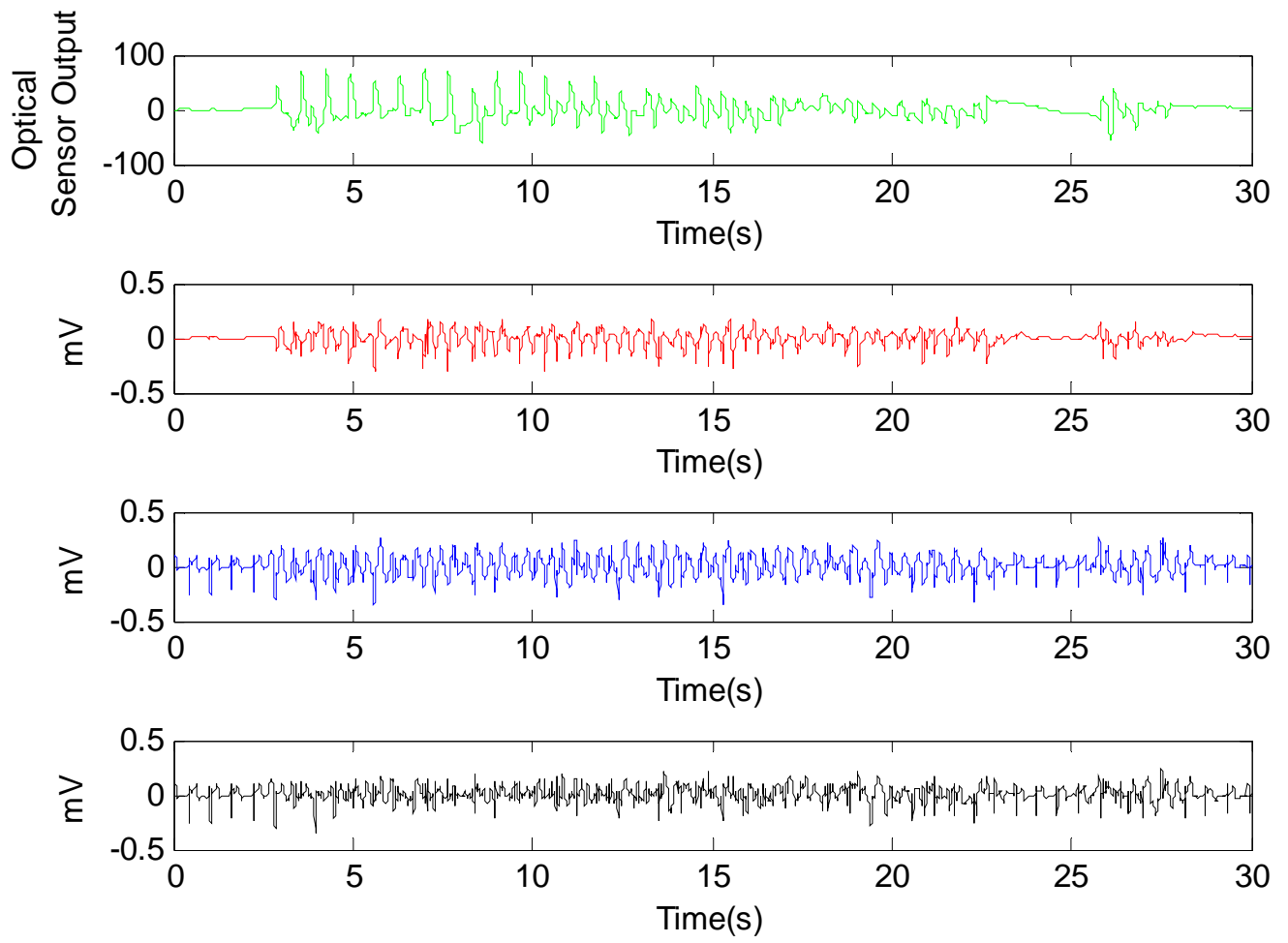

Ambulatory ECG motion artifact reduction (recording 15: subject is running); top trace: optical sensor output; second trace: artifacts estimated by the adaptive filter; third trace: ECG signal corrupted by artifacts; bottom trace: ECG signal after artifact reduction 


\section{Bibliography}

Akay, M., Akay, Y. M., Welkowitz, W., Semmlow, J.L., and Kostis, J. B., "Application of Adaptive Filters to Noninvasive Acoustical Detection of Coronary Occlusions before and after Angioplasty", IEEE Transactions on Biomedical Engineering, Vol. 39, pp. 176-184, Feb 1992.

American Heart Association, "Heart Disease and Stroke Statistics — 2005 Update", 2005.

American Heart Association, "International Cardiovascular Disease Statistics", 2005.

Appel, L.J., Robinson, K.A., Guallar, E., Erlinger, T., Masood, S.O., Jehn, M., Fleisher, L., Powe, N.R., and Bass, E.B., "Utility of Blood Pressure Monitoring Outside of the Clinic Setting”, Evidence Report/Technology Assessment No. 63 (Prepared by the Johns Hopkins Evidence-based Practice Center under Contract No 290-97-006). AHRQ Publication No. 03-E004. Rockville, MD: Agency for Healthcare Research and Quality. November 2002.

Asada, H. H., Cho, K. J., and Au, S. K. W., "Smart Moles: A Hybrid Wireless Stethoscope / Telephone System Using Skin-Attached Passive Microphones for Continuous Health Monitoring and Voice Communication", Progress Report No. 3-1, MIT Home Automation and Healthcare Consortium, April 2001.

Asada, H.H. and Awad, E., "The Doppler Necklace: a Wearable and Noninvasive Ultrasound Sensor for Continuous Monitoring of Blood Flow in the Common Carotid Artery", The Home Automation and Healthcare Consortium, Progress Report No. 2-3, March 31, 1999.

Asada, H.H., Shaltis, P., Reisner, A., Sokwoo Rhee, and Hutchinson, R.C., "Mobile Monitoring with Wearable Photoplethysmographic Biosensors", IEEE Engineering in Medicine and Biology Magazine, Vol. 22, No. 3, pp. 28 - 40, May-June 2003.

Asada, H.H.; Hong-Hui Jiang; Gibbs, P.; “Active Noise Cancellation Using MEMS Accelerometers for Motion-Tolerant Wearable Bio-Sensors", Engineering in Medicine and Biology Society (EMBC), Proceedings of the 26th Annual International Conference, Vol. 1, pp. 2157 - 2160, 2004.

Avago Technologies, “Optical Sensors - Agilent ADNS-3040 Ultra Low-Power Optical Mouse Sensor”, Online 2005, http://www.avagotech.com/products/document-results.jsp?catId=5233, last accessed December 14, 2005. 
Awad, E. and Asada, H.H., "The Doppler Necklace: a Wearable and Noninvasive Ultrasound Sensor for Continuous Monitoring of Blood Flow in the Common Carotid Artery", 21st Annual Conference and the 1999 Annual Fall Meeting of the Biomedical Engineering Society, Proceedings of the First Joint, Vol. 2, pp. 795, October 13-16, 1999.

Branche, P.C., Johnston, W.S., Pujary, C.J., and Mendelson, Y., "Measurement Reproducibility and Sensor Placement Considerations in Designing a Wearable Pulse Oximeter for Military Applications", Proceedings of the IEEE 30th Annual Northeast Bioengineering Conference, pp. 216 - 217, April 17-18, 2004.

Braun.com, "Blood Pressure Measurement", Online 2000, http://www.braun.com/medical/bloodpressure/downloads/measurement.Downloa dPara.0001.File0.tmp.pdf, last accessed March 22, 2006.

Burbank, D.P., and Webster, J.G., "Reducing Skin Potential Motion Artifact by Skin Abrasion", Medical \& Biological Engineering \& Computing, Vol. 16, pp. 31-38, 1978.

Burbank, D.P., and Webster, J.G., "Reducing Skin Potential Motion Artifact by Skin Abrasion", Medical \& Biological Engineering \& Computing, Vol. 16, pp. 31-38, 1978.

Cardionet, "How It Works", Online 2002, http://www.cardionet.com/how.html, last accessed March 22, 2006.

Centers for Disease Control and Prevention (CDC), "State-Specific Mortality from Sudden Cardiac Death - United States, 1999”, Morbidity and Mortality Weekly Report (MMWR), Vol. 51, No. 6, February 15, 2002.

Charuvastra, E., "Transtelephonic Cardiac Event Recording for Arrhythmia Surveillance", Congenital Heart Information Network, Online March 11, 1998, http://tchin.org/resource_room/c_art_07.htm, last accessed March 22, 2006.

Chen, Y., Li, L., Hershler, C. and Dill, R.P., "Continuous Non-invasive Blood Pressure Monitoring Method and Apparatus", United States Patent 6599251, July 29, 2003.

Chen, Y., Li, L., Hershler, C. and Dill, R.P., "Continuous Non-invasive Blood Pressure Monitoring Method and Apparatus", United States Patent 6893401, May $17,2005$.

Cheong, P.Y.S., and Smith, P.R., "An Overview of Non-contact Photoplethysmography", Electronic Systems and Control Division Research Conference, Department of Electronic and Electrical Engineering, Loughborough University, LE11 3TU, UK, pp. 57-59, September, 2003. 
Chia, Chee W., Greenwald, Scott D., and Mark, Roger G., "A Comparison of Human Experts and Computer Algorithms in Detecting and Classifying Beats in Noise-Corrupted Electrocardiograms", Computers in Cardiology 1991, pp. 465468, May, 1991.

Chittenden, C.T., and Weaver, C.S., "Blood Pressure Measurement Artifact Rejection in Noisy Environments", Proceedings of the Annual International Conference of the IEEE Engineering in Medicine and Biology Society, Vol. 1, pp. 78 - 79, November 4-7, 1988.

Cho, K. J., and Asada, H.H., "Wireless, Battery-less Stethoscope for Wearable Health Monitoring", Proceedings of the IEEE 28th Annual Northeast Bioengineering Conference, pp. 187 - 188, 2002.

Cleveland Clinic Heart Center, “Ambulatory Monitors", Online December 2003, http://www.clevelandclinic.org/heartcenter/pub/guide/tests/electrocard/ambmonit or.htm, last accessed March 22, 2006.

Colin, "Tonometry", http://www.colin-europe.com/pages/tonometry.html, last accessed March 22, 2006.

Cristalli, C., Neuman, M.R., and Ursino, M., "Studies on Soft Tissue Pressure Distribution in the Arm During Non-Invasive Blood Pressure Measurement", Engineering Advances: New Opportunities for Biomedical Engineers, Proceedings of the 16th Annual International Conference of the IEEE Engineering in Medicine and Biology Society, Vol.1, pp. 41 - 42, November 3-6, 1994.

Cybulski, G., “Ambulatory Impedance Cardiography: New Possibilities”, Journal of Applied Physiology, Vol. 88, pp. 1509-1510, 2000.

Cybulski, G., Ksiazkiewicz, A., Lukasik, W., Niewiadomski, W., and Palko, T., "Ambulatory Monitoring Device for Central Hemodynamic and ECG Signal Recording on PCMCI Flash Memory Cards", Computers in Cardiology 1995, pp. 505 - 507, September 10-13, 1995.

Cybulski, G., Ziolkowska, E., Kodrzycka, A., Niewiadomski, W., Sikora, K., Ksiazkiewicz, A., Lukasik, W., and Palko, T., "Application of Impedance Cardiography Ambulatory Monitoring System for Analysis of Central Hemodynamics in Healthy Man and Arrhythmia Patients", Computers in Cardiology 1997, pp. 509 - 512, September 7-10, 1997.

Cybulski, G., Ziolkowska, E., Ksiazkiewicz, A., Lukasik, W., Niewiadomski, W., Kodrzycka, A., and Palko, T., "Application of Impedance Cardiographic Ambulatory Monitoring Device for Analysis of Central Hemodynamic Variability in Atrial Fibrillation", Computers in Cardiology 1999, pp. 563 - 566, September 26-29, 1999. 
Devlin, P.H., Mark, R.G., and Ketchum, J.W., "Detection of Electrode Motion Noise in ECG Signals by Monitoring Electrode Impedance", Computers in Cardiology, pp. 51-56, 1984.

El-Asir, B., and Mayyas, K., "Multiresolution Analysis of Heart Sounds using Filter Banks", Information Technology Journal, Vol. 3, No. 1, pp. 36-43, 2004.

Eveloy, E., Liu, Y., and Pecht, M., "Heart Sound Measurement and Analysis in Cardiovascular Disease Assessment", Surface Mount Technology Association (SMTA), Proceedings of the SMTA Medical Electronics Symposium, Minneapolis, MN, April 25-27, 2005.

Fearnley, S.J., "Pulse Oximetry", Update in Anaesthesia, Issue 5, Article 2, 1995.

Feldman, C., Hubelbank, M., Hassagee, C., Kotilanion, P., “A New Electrode System for Automated ECG Monitororing”, Computers in Cardiology, pp. 285$288,1979$.

Friesen, G. M., Jannett, T. C., Jadallah, M. A., Yates, S. L., Quint, S. R., Nagle, H. T., "Comparison of the Noise Sensitivity of Nine QRS Detection Algorithms", IEEE Transactions on Biomedical Engineering, Vol. 37, No. 1, pp. 85-98, Jan, 1990.

Georgia Tech, "George Tech Wearable Motherboard: The Intelligent Garment for the 21 st Century", http://www.gtwm.gatech.edu/, last accessed March 22, 2006.

Grimnes, S., and Martinsen, O.G., Bioimpedance \& Bioelectricity Basics, Academic Press, 2000.

Grossman, P., “Ambulatory Cardiovascular, Ventilatory and Activity Monitoring: Progress and Potential for Assessing Real-Life Physiology in Health and Disease", http://www.vivometrics.com/site/res_whitepapers.html, last accessed March 22, 2006.

Guyton, A.C., and Hall, J.E., Textbook of Medical Physiology, tenth edition, W.B. Saunders Company, 2000.

Hall, L. T., Maple, J. L., Agzarian, J., and Abbott, D., "Sensor System for Heart Sound Biomonitor”, Microelectronic Journal, Vol. 31, pp. 583 - 592, 2000.

Hamilton, P.S., and Curley, M.G., "Adaptive Removal of Motion Artifact", Engineering in Medicine and Biology society, Proceedings of the 19th Annual International Conference, Vol. 1, October 30 - November 2, pp. 297 - 299, 1997. 
Hamilton, P.S., Curley, M., and Aimi, R., "Effect of Adaptive Motion-Artifact Reduction on QRS Detection", Biomedical Instrumentation and Technology, Vol. 34, pp. 197-202, 2000a.

Hamilton, P.S., Curley, M.G., Aimi, R.M., and Sae-Hau, C., "Comparison of Methods for Adaptive Removal of Motion Artifact", Computers in Cardiology, September 24-27, pp. $383-386,2000$ b.

Hamilton, P.S., Curley, M.G., and Aimi, R.M., "Apparatus and Method for Reduction of Motion Artifact”, U.S. patent 5978693, November 2, 1999.

Harland, C.J., Clark, T.D., and Prance, R.J., "Electrical Potential Probes - New Directions in the Remote Sensing of the Human Body", Measurement Science and Technology, Vol. 13, pp. 163-169, 2002.

Harland, C.J., Clark, T.D., and Prance, R.J., "High Resolution Ambulatory Electrocardiographic Monitoring Using Wrist-Mounted Electric Potential Sensors", Measurement Science and Technology, Vol. 14, pp. 923-928, 2003.

Haykin, S., Adaptive Filter Theory, Fourth Edition, Prentice Hall, 2001.

Hill, E., and Stoneham, M.D., "Practical Applications of Pulse Oximetry", Update in Anaesthesia, Issue 11, Article 4, 2000.

Johnson, B.D., "Sensor Tracks Soldiers' Vital Signs”, Photonics Spectra, pp. 5253, January 2003.

Kadish, A.H., "ACC/AHA Clinical Competence Statement on Electrocardiography and Ambulatory Electrocardiography", Journal of the American College of Cardiology, Vol. 38, No. 7, pp. 2091-2100, 2001.

Kim, Y., and Jong, M. C., "Development of Wireless Bio-Telemetry System Using FM Stereo Method for Exercising Rehabilitation Patients", Proceedings of the 23rd Annual International Conference of the IEEE Engineering in Medicine and Biology Society, Vol. 4, pp. 3338-3340, 2001.

Kingsley, S.A., Sriram, S., Pollick, A., and Marsh, J., "PhotrodeTM Optical Sensor for Electrophysiological Monitoring", Aviation, Space, and Environmental Medicine, Vol. 74, No. 11, pp. 1215-1216, November, 2003.

Kingsley, S.A., Sriram, S., Pollick, A., and Marsh, J., "Revolutionary Optical Sensor for Physiological Monitoring in the Battlefield", The International Society for Optical Engineering, Sensors, and Command, Control, Communications, and Intelligence (C31) Technologies for Homeland Security and Homeland Defense III, pp. 68-77, April 12-16, 2004. 
Kingsley, S.A., Sriram, S., Pollick, A., Caldwell, J., Pearce, F., and Sing, H., "Physiological Monitoring with High-Impedance Optical Electrodes (PhotrodesTM)", 23rd Annual Army Science Conference, December 2-5, 2002.

Kmom, "The Importance of Large Blood Pressure Cuffs", Online April 2003, http://www.plus-size-pregnancy.org/lgbpcuffs.htm, last accessed March 22, 2006.

Kowey, P.R., and Kocovic, D.Z., “Ambulatory Electrocardiographic Recording”, Circulation, Vol. 108, pp. e31-e33, 2003.

LifeWatch, "WristRecorder Plus", Online 2004, http://www.lifewatchinc.com/WristRecorder-Plus.asp, last accessed March 22, 2006.

Lin, C., Liu, S., Wang, J., and Wen, Z., "Reduction of Interference in Oscillometric Arterial Blood Pressure Measurement Using Fuzzy Logic", IEEE Transactions on Biomedical Engineering, Vol. 50, No. 4, pp. 432 - 441, April 2003.

Liu, Y. and Pecht, M., "Reduction of Skin Stretch Induced Motion Artifacts in Electrocardiogram Monitoring Using Adaptive Filtering", 28th International Conference of the IEEE Engineering in Medicine and Biology Society: Engineering Revolution In BioMedicine, New York, NY, August 31-September 3, 2006.

Liu, Y., Eveloy, V. and Pecht, M., "Non-invasive Electrocardiogram and Blood Pressure Monitors for Cardiovascular Disease", 38th International Symposium on Microelectronics, Sensor and MEMS Packaging, pp. 66-74, Philadelphia, PA, September 25-29, 2005.

Luo, S., and Tompkins, W.J., "Experimental Study: Brachial Motion Artifact Reduction in the ECG”, Computers in Cardiology, pp. 33 - 36, Sept. 10-13, 1995.

Marchiando, R.J., and Elston, M.P., "Automated Ambulatory Blood Pressure Monitoring: Clinical Utility in the Family Practice Setting", American Family Physician, Vol. 67, No. 11, pp. 2343-2350, June 2003.

Mathie, M.J., Coster, A.C.F., Lovell, N.H., and Celler, B.G., “Accelerometry: Providing an Integrated, Practical Method for Long-Term, Ambulatory Monitoring of Human Movement", Physiological Measurement, Vol. 24, pp. R1R20, 2004.

McGrath, B.P., "Ambulatory Blood Pressure Monitoring”, The Medical Journal of Australia, Vol. 176, pp. 588-592, June 17, 2002. 
MedFirst Healthcare Supply, Inc., "Holters",

http://www.medfirstonline.com/showitems.cfm?Product_Group=Holters, last accessed March 22, 2006.

Mendoza, G.G., and Tran, B.Q., "In-Home Wireless Monitoring of Physiological Data for Heart Failure Patients", Proceedings of the 24th Engineering in Medicine and Biology Annual Conference and the Annual Fall Meeting of the Biomedical Engineering Society (EMBS/BMES), Vol. 3, pp. 1849- 1850, 2002.

MIT Media Lab, "MIThril", http://www.media.mit.edu/wearables/mithril/, last accessed March 22, 2006.

Molhoek, P.G., Wesseling, K.H., Arntzenius, A.C., Settels, J.J.M., Vollenhoven, E.V., Weeda, H.W.A., "Evaluation of the Penaz Servo-Plethysmo-Manometer for Continuous, Noninvasive Measurement of Finger Blood Pressure", Blood Pressure Measurement and Systemic Hypertension: 2nd Einthoven Meeting on Past and Present Cardiology, Edited by Arntzenius, A.C., Dunning, A.J., and Snellen, H.A., pp. 157-162, 1981.

Mona-System Ltd., "ECG - Watch: an ECG Event Recorder", http://www.monasystem.hu/html/angolmerlin.html, last accessed March 22, 2006.

Morucci, J.P., Valentinuzzi, M.E., Rigaud, B., Felice, C.J., Chauveau, N., and Marsili, P.M., "Bioelectrical Impedance Techniques in Medicine", Critical Reviews in Biomedical Engineering, Vol. 24, Issues 4-6, pp. 223-677, 1996.

Nakonezny, P.A., Kowalewski, R.B., Ernst, J.M., Hawkley, L.C., Lozano, D.L., Litvack, D.A., Berntson, G.G., Sollers, J.J., Kizakevich, P., Cacioppo, J.T., and Lovallo, W.R., "New Ambulatory Impedance Cardiography Validated Against the Minnesota Impedance Cardiograph", Psychophysiology, Vol. 38, pp. 465-473, 2001.

Ng, K.G., and Small, C.F., "Changes in Oscillometric Pulse Amplitude Envelope with Cuff Size: Implications for Blood Pressure Measurement Criteria and Cuff Size Selection", Journal of Biomedical Engineering, Vol. 14, No. 4, pp. 279-282, July, 1993.

O’Brien, E., Coats, A., Owens, P., Petrie, J., Padfield, P.L., Little, W.A., Swiet, M., and Mee, F., "Use and Interpretation of Ambulatory Blood Pressure Monitoring: Recommendations of the British Hypertension Society", British Medical Journal, Vol. 320, pp. 1128-1134, April 2000.

Odman, S., "Potential and Impedance Variations Following Skin Deformation", Medical \& Biological Engineering \& Computing, Vol. 19, pp. 271-278, 1981. 
Odman, S., "On the Spread of Deformation Potentials in the Skin", Medical \& Biological Engineering \& Computing, Vol. 20, pp. 451-456, 1982.

Odman, S., and Oberg, P.A., "Movement-Induced Potentials in Surface Electrodes", Medical \& Biological Engineering \& Computing, Vol. 20, pp. 159$166,1982$.

Paradiso, R., Loriga, G., and Taccini, N., “A Wearable Health Care System Based on Knitted Integrated Sensors", IEEE Transactions on Information Technology in Biomedicine, Vol. 9, No. 3, pp. 337-344, September, 2005.

Pavlopoulos, S. A., Stasis, A. CH and Loukis, E. N., "A Decision Tree-Based Method for the Differential Diagnosis of Aortic Stenosis from Mitral Regurgitation using Heart Sounds", Biomedical Engineering Online, Vol. 3, No. 21, 2004.

Pecht, M. and Liu, Y., "Sensor Systems and Methods for Reducing Motion Artifact", reported to the Office of Technology Commercialization, University of Maryland, December, 2005, Ref. No. PS-2005-110.

Penaz, J., "Photoelectric Measurement of Blood Pressure, Volume and Flow in the Finger", Digest of the 10th International Conference on Medical and Biological Engineering, Vol. 1, pp. 104, 1973.

Petersen, S., Peto, V., Rayner, M., Leal, J., Luengo-Fernandez R., and Gray, A., "European Cardiovascular Disease Statistics - 2005 Edition”, 2005.

Peterson, W.D., "Phoenix Ambulatory Blood Pressure Monitor Project Subproject: Impedance Plethysmograph Sensor", November 21, 2004,

http://www.phoenix.tc-

ieee.org/009_Impedance_Plethysmograph_Sensor/Phoenix_Plethysmograph.htm, last accessed March 22, 2006.

Pouladian, M. and Golpayegani, M.R.H., "Noninvasive Detection of Atherosclerosis by Arterio-Oscillo-Gram", Proceedings of the 25th Annual International Conference of the IEEE Engineering in Medicine and Biology Society, Vol.1, pp. 272 - 275, September 17-21, 2003.

Pouladian, M., Golpayegani, M.R., Tehrani-Fard, A.A., and Bubvay-Nejad, M., "Noninvasive Detection of Coronary Artery Disease by Arterio-Oscillo-Graphy", IEEE Transactions on Biomedical Engineering, Vol. 52, No. 4, pp. 743-747, April 2005.

Prance, R.J., Debray, A., Clark, T.D., Prance, H., Nock, M., Harland, C. J., “An Ultra-Low-Noise Electrical-Potential Probe for Human-Body Scanning", Measurement Science and Technology, Vol. 11, pp. 291-297, 2000. 
Protocol Systems, Inc., "Noninvasive Blood Pressure Measurement and Motion Artifact: A Comparative Study", December 3, 1998, http://www.monitoring.welchallyn.com/pdfs/smartcuf_whitepaper.pdf, last accessed March 22, 2006.

Raya, M.A.D., and Sison, L.G., "Adaptive Noise Canceling of Motion Artifact in Stress ECG Signals Using Accelerometer", Engineering in Medicine and Biology (EMBS/BMES), Proceedings of the Second Joint of the 24th Annual Conference and the Annual Fall Meeting, Vol. 2, pp. 1756 - 1757, October 23-26, 2002.

Raza, S.B., Patterson, R.P., and Wang, L., "Removal of Respiratory Artifacts from Cardiogenic Impedance Signal by Digital Filtering", Proceedings of the 11th Annual International Conference of the IEEE Engineering in Medicine and Biology Society, Vol.2, pp. 634 - 635, November 9-12, 1989.

Rhee, S., Yang, B., and Asada, H.H., “Artifact-Resistant Power-Efficient Design of Finger-Ring Plethysmographic Sensors", IEEE Transactions on Biomedical Engineering, Vol. 48, No. 7, pp. 795 - 805, July 2001.

Rhee, S., Yang, B., Chang, K., and Asada, H.H., "The Ring Sensor: a New Ambulatory Wearable Sensor for Twenty-Four Hour Patient Monitoring", Proceedings of the 20th Annual International Conference of the IEEE Engineering in Medicine and Biology Society, Vol. 4, pp. 1906 - 1909, Oct. 29 - Nov. 1, 1998.

Ruffini, G., Dunne, S., Farres, E., Marco-Pallares, J., Ray, C., Mendoza, E., Silva, R., and Grau, C., "A Dry Electrophysiology Electrode Using CNT Arrays", Submitted to Sensors and Actuators, Proceedings of Eurosensors XIX, Barcelona, Spain, 2005, http://arxiv.org/abs/physics/0510145, last accessed March 22, 2006.

Sastry, U.R.B., "An Overview of Worldwide Development in Smart Textiles", Technical Textile International, Vol. 13, No. 4, pp. 31-35, 2004.

Sato, T., Nishinaga, M., Kawamoto, A., Ozawa, T., and Takatsuji, H., "Accuracy of a Continuous Blood Pressure Monitor Based on Arterial Tonometry", Hypertension, Vol. 21, pp. 866-874, 1993.

Scanlon, M. V., "Acoustic Sensor for Health Status Monitoring", Proceeding of IRIS Acoustic Seismis, Vol. 2, pp. 205-222, 1998.

Scilingo, E.P., Gemignani, A., Paradiso, R., Taccini, N., Ghelarducci, B., and De Rossi, D., "Performance Evaluation of Sensing Fabrics for Monitoring Physiological and Biomedical Variables", IEEE Transactions on Information Technology in Biomedicine, Vol. 9, No. 3, pp. 337-344, September, 2005.

Selig, M. B., "Stethoscopic and Phonoaudio Devices: Historical and Future Perspective", American Heart Journal, pp. 262-268, July 1993. 
Shaltis, P., Reisner, A., and Asada, H., "A Hydrostatic Pressure Approach to Cuffless Blood Pressure Monitoring", Proceedings of the 26th Annual International Conference of the Engineering in Medicine and Biology Society (EMBC), Vol. 1, pp. 2173 - 2176, 2004.

Sherwood, A., Mcfetridge, J., and Hutcheson, J.S., "Ambulatory Impedance Cardiography: A Feasibility Study", Journal of Applied Physiology, Vol. 85, pp. 2365-2369, 1998.

Smith, M., "Rx for ECG Monitoring Artifact", Critical Care Nurse, Vol. 4, No. 1, pp. 64-66, 1984.

Spigulis, J., Erts, R., and Rubins, U., "Micro-Circulation of Skin Blood: Optical Monitoring by Advanced Photoplethysmography Technique", University of Latvia, Physics Department and IAPS, http://home.lanet.lv/ spigulis/mnmfinal.htm, last accessed March 22, 2006.

Spigulis, J., Ozols, M., Erts R., and Prieditis, K., "A Portable Device for Optical Assessment", University of Latvia, Physics Department and IAPS, http://home.lanet.lv/ spigulis/PPG-AOMD-3.htm, last accessed March 22, 2006.

Staessen, J.A., Fagard, R., and Thijs, L., "A Consensus View on the Technique of Ambulatory Blood Pressure Monitoring”, Hypertension, Vol. 26, pp. 912-918, 1995.

Tam, R. W. and Webster, J.G., "Minimizing Electrode Motion Artifact by Skin Abrasion", IEEE Transactions on Biomedical Engineering, BME-29, pp. 134-139, 1977.

Tong, D.A., "Electrode Systems and Methods for Reducing Motion Artifact", U.S. patent 6912414, June 28, 2005.

Tong, D.A., Bartels, K.A., Honeyager, K.S., "Adaptive Reduction of Motion Artifact in the Electrocardiogram", Engineering in Medicine and Biology (EMBS/BMES), Proceedings of the Second Joint of the 24th Annual Conference and the Annual Fall Meeting, Vol. 2, pp. 1403 - 1404, October 23-26, 2002.

Tong, D.A., "Investigation into Electrocardiogram Motion Artifact Reduction, 109191", Southwest Research Institute, April 01, 2000, http://www.swri.edu/3pubs/IRD2000/10-9191.htm, last accessed July 21, 2005.

Veraart, J.C.J.M., Joost Van Der Kley, A.M., and Martino Neumann, H.A., "Digital Photoplethysmography and Light Reflection Rheography", Journal of Dermatologic Surgery and Oncology, Vol. 20, No. 7, pp. 470-473, July, 1994. 
Virginia Mason Medical Center, "Holter Monitors", Online July 19, 2000, http://www.virginiamason.org/dbCardiology/sec358.htm, last accessed March 22, 2006.

VivoMetrics, "How It Works", http://www.vivometrics.com/site/system_howitworks.html, last accessed March 22, 2006.

Wade, E. and Asada, H.H.; "Cable-Free Wearable Sensor System Using a DC Powerline Body Network in a Conductive Fabric Vest", Conference Proceedings of the 26th Annual International Conference of the Engineering in Medicine and Biology Society (EMBC), Vol. 2, pp. 5376 - 5379, September 1-5, 2004.

Wang, H., and Wang, L. Y., "Continuous Intro-Operative Respiratory Auscultation in Anesthesia", Proceedings of IEEE Sensors, Vol. 2, pp. 1002 1005, October 2003.

Webster, J.G., "Reducing Motion Artifacts and Interference in Biopotential Recording", IEEE Transactions on Biomedical Engineering, Vol. BME-31, No. 12, pp. 823-826, 1984.

Webster, J.G., Medical Instrumentation: Application and Design, third edition, John Wiley \& Sons, Inc., 1998.

Welch, J., Guilak, F., and Baker, S.D., "A Wireless ECG Smart Sensor for Broad Application in Life Threatening Event Detection", Engineering in Medicine and Biology Society (EMBC), Proceedings of the 26th Annual International Conference, Vol. 2, pp. 3447-3449, 2004.

Welch, J.P., DeCesare, R., and Hess, D., "Pulse Oximetry: Instrumentation and Clinical Applications”, Respiratory Care, Vol. 35, No. 6, pp. 584-597, June 1990.

Wiese, S.R., Anheier, P., Connemara, R.D., Mollner, A.T., Neils, T.F., Kahn, J.A., and Webster, J.G., "Electrocardiographic Motion Artifact Versus Electrode Impedance", IEEE Transactions on Biomedical Engineering, Vol. 52, No. 1, pp. $136-139,2005$.

Wood, J.C., and Barry, D.T., "Time- Frequency Analysis of the First Heart Sound", IEEE Engineering in Medicine and Biology Magazine, Vol. 14 , No. 2 , pp. 144-151, March-April 1995.

Xu, J., Durand, L. G., and Pibarot, P., "Extraction of the Aortic and Pulmonary Components of the Second Heart Sound using a Nonlinear Transient Chirp Signal Model”, IEEE Transactions on Biomedical Engineering, Vol. 48, No. 3, pp. 277 283, March 2001. 
Yamashita, M., Shimizu, K., and Matsumoto, G., "Development of a Ring-Type Vital Sign Telemeter", Biotelemetry XIII, Proceedings of the Thirteenth International Symposium on Biotelemetry, March 26-31, 1995.

Yang, B., Rhee, S., and Asada, H.H., “A Twenty-Four Hour Tele-Nursing System Using a Ring Sensor", Proceedings of 1998 IEEE International Conference on Robotics and Automation, Vol. 1, pp. 387 - 392, May 16-20, 1998.

Zimetbaum, P.J., and Josephson, M.E., "The Evolving Role of Ambulatory Arrhythmia Monitoring in General Clinical Practice", Annals of Internal Medicine, Vol. 130, pp. 848-856, 199

Thakor, N.V., and Webster, J. G., "The origin of skin potential and its variations", Proceedings of the 31st Annual Conference on Engineering in Biology and Medicine, Vol. 20, pp. 212, 1978.

de Talhouet, H., and Webster, J. G., "The Origin of Skin-Stretch-Caused Motion Artifacts under Electrodes", Physiological Measurement, Vol. 17, pp. 81-93, 1996.

Thakor, N.V., and Zhu, Y., "Applications of Adaptive Filtering to ECG Analysis: Noise Cancellation and Arrhythmia Detection", IEEE Transactions on Biomedical Engineering, Vol. 38, No. 8, pp. 785-794, Aug, 1991.

Xue, Q., Hu, Y. H., and Tompkins, W. J., "Neural-Network-Based Adaptive Matched Filtering for QRS Detection", IEEE Transactions on Biomedical Engineering, Vol. 39, No. 4, pp. 317-329, 1992.

Kiryu, T., Kaneko, H., and Saitoh, Y., "Motion Artifact Elimination Using Fuzzy Rule Based Adaptive Nonlinear Filter", IEICE Transactions on Fundamentals of Electronics, Communications and Computer Sciences, Vol. E77-A, No. 5, pp. 833-838, May, 1994.

Widrow, B., Glover, J.R., McCool, J.M., Kaunitz, J., Williams, C.S., Hearn, R.H., Zeidler, J.R., Dong, E., and Goodlin, R.C., "Adaptive Noise Canceling: Principles and Applications", Proceedings of the IEEE, Vol. 63, No. 12, pp. 1692-1716, 1975. 